\title{
British Thoracic Society guidelines for the management of community acquired pneumonia in adults: update 2009
}

\author{
W S Lim, S V Baudouin, R C George, A T Hill, C Jamieson, I Le Jeune, J T Macfarlane, \\ R C Read, H J Roberts, M L Levy, M Wani, M A Woodhead, Pneumonia Guidelines \\ Committee of the BTS Standards of Care Committee
}

- Full search strategies for each database are published online only at http://thorax.bmi.com/ content/vol64/issueSuppllII

Correspondence to: Dr W S Lim, Respiratory Medicine, Nottingham University Hospitals, David Evans Building, Hucknall Road, Nottingham NG5 1PB, UK; weishen.lim@nuh.nhs.uk

Received 11 June 2009 Accepted 6 July 2009

\section{SYNOPSIS OF RECOMMENDATIONS}

A summary of the initial management of patients admitted to hospital with suspected community acquired pneumonia (CAP) is presented in fig 8 . Tables 4 and 5, respectively, summarise (1) the relevant microbiological investigations and (2) empirical antibiotic choices recommended in patients with CAP.

\section{Investigations (Section 5)}

When should a chest radiograph be performed in the community?

1. It is not necessary to perform a chest radiograph in patients with suspected CAP unless:

- The diagnosis is in doubt and a chest radiograph will help in a differential diagnosis and management of the acute illness. [D]

- Progress following treatment for suspected CAP is not satisfactory at review. [D]

- The patient is considered at risk of underlying lung pathology such as lung cancer. [D]

When should a chest radiograph be performed in hospital?

2. All patients admitted to hospital with suspected CAP should have a chest radiograph performed as soon as possible to confirm or refute the diagnosis. [D] The objective of any service should be for the chest radiograph to be performed in time for antibiotics to be administered within $4 \mathrm{~h}$ of presentation to hospital should the diagnosis of CAP be confirmed.

When should the chest radiograph be repeated during recovery?

3. The chest radiograph need not be repeated prior to hospital discharge in those who have made a satisfactory clinical recovery from CAP. [D]

4. A chest radiograph should be arranged after about 6 weeks for all those patients who have persistence of symptoms or physical signs or who are at higher risk of underlying malignancy (especially smokers and those aged $>50$ years) whether or not they have been admitted to hospital. [D]

5. Further investigations which may include bronchoscopy should be considered in patients with persisting signs, symptoms and radiological abnormalities at around 6 weeks after completing treatment. [D]
6. It is the responsibility of the hospital team to arrange the follow-up plan with the patient and the general practitioner for those patients admitted to hospital. [D]

What general investigations should be done in the community?

7. General investigations are not necessary for the majority of patients with CAP who are managed in the community. [C] Pulse oximeters allow for simple assessment of oxygenation. General practitioners, particularly those working in out-of-hours and emergency assessment centres, should consider their use. [D]

8. Pulse oximetry should be available in all locations where emergency oxygen is used. [D]

What general investigations should be done in a patient admitted to hospital?

9. All patients should have the following tests performed on admission:

- Oxygenation saturations and, where necessary, arterial blood gases in accordance with the BTS guideline for emergency oxygen use in adult patients. $[\mathrm{B}+]$

- Chest radiograph to allow accurate diagnosis. $[\mathrm{B}+]$

- Urea and electrolytes to inform severity assessment. $[\mathrm{B}+]$

- C-reactive protein to aid diagnosis and as a baseline measure. $[\mathrm{B}+]$

- Full blood count. [B-]

- Liver function tests. [D]

Why are microbiological investigations performed?

10. Microbiological tests should be performed on all patients with moderate and high severity CAP, the extent of investigation in these patients being guided by severity. [D]

11. For patients with low severity CAP the extent of microbiological investigations should be guided by clinical factors (age, comorbid illness, severity indicators), epidemiological factors and prior antibiotic therapy. $[\mathrm{A}-]$

12. Where there is clear microbiological evidence of a specific pathogen, empirical antibiotics should be changed to the appropriate 
pathogen-focused agent unless there are legitimate concerns about dual pathogen infection. [D]

\section{What microbiological investigations should be performed in the community?}

13. For patients managed in the community, microbiological investigations are not recommended routinely. [D]

14. Examination of sputum should be considered for patients who do not respond to empirical antibiotic therapy. [D]

15. Examination of sputum for Mycobacterium tuberculosis should be considered for patients with a persistent productive cough, especially if malaise, weight loss or night sweats, or risk factors for tuberculosis (eg, ethnic origin, social deprivation, elderly) are present. [D]

16. Urine antigen investigations, PCR of upper (eg, nose and throat swabs) or lower (eg, sputum) respiratory tract samples or serological investigations may be considered during outbreaks (eg, Legionnaires' disease) or epidemic mycoplasma years, or when there is a particular clinical or epidemiological reason. [D]

\section{What microbiological investigations should be performed in hospital? Blood cultures}

17. Blood cultures are recommended for all patients with moderate and high severity CAP, preferably before antibiotic therapy is commenced. [D]

18. If a diagnosis of CAP has been definitely confirmed and a patient has low severity pneumonia with no comorbid disease, blood cultures may be omitted. $[\mathrm{A}-]$

\section{Sputum cultures}

19. Sputum samples should be sent for culture and sensitivity tests from patients with CAP of moderate severity who are able to expectorate purulent samples and have not received prior antibiotic therapy. Specimens should be transported rapidly to the laboratory. $[\mathrm{A}-]$

20. Culture of sputum or other lower respiratory tract samples should also be performed for all patients with high severity CAP or those who fail to improve. $[\mathrm{A}-]$

21. Sputum cultures for Legionella spp should always be attempted for patients who are legionella urine antigen positive in order to provide isolates for epidemiological typing and comparison with isolates from putative environmental sources. [D]

\section{Sputum Gram stain}

22. Clinicians should establish with local laboratories the availability or otherwise of sputum Gram stain. Where this is available, laboratories should offer a reliable Gram stain for patients with high severity CAP or complications as occasionally this can give an immediate indicator of the likely pathogen. Routine performance or reporting of sputum Gram stain on all patients is unnecessary but can aid the laboratory interpretations of culture results. $[\mathrm{B}-]$

23. Samples from patients already in receipt of antimicrobials are rarely helpful in establishing a diagnosis. [B-]

24. Laboratories performing sputum Gram stains should adhere to strict and locally agreed criteria for interpretation and reporting of results. $[\mathrm{B}+]$

\section{Other tests for Streptococcus pneumoniae}

25. Pneumococcal urine antigen tests should be performed for all patients with moderate or high severity CAP. [A-]

26. A rapid testing and reporting service for pneumococcal urine antigen should be available to all hospitals admitting patients with CAP. $[\mathrm{B}+]$

\section{Tests for Legionnaires' disease}

27. Investigations for legionella pneumonia are recommended for all patients with high severity CAP, for other patients with specific risk factors and for all patients with CAP during outbreaks. [D]

28. Legionella urine antigen tests should be performed for all patients with high severity CAP. $[\mathrm{B}+]$

29. A rapid testing and reporting service for legionella urine antigen should be available to all hospitals admitting patients with CAP. $[\mathrm{B}+]$

30. As the culture of legionella is very important for clinical reasons and source identification, specimens of respiratory secretions, including sputum, should be sent from patients with high severity CAP or where Legionnaires' disease is suspected on epidemiological or clinical grounds. [D] The clinician should specifically request legionella culture on laboratory request forms.

31. Legionella cultures should be routinely performed on invasive respiratory samples (eg, obtained by bronchoscopy) from patients with CAP. [D]

32. For all patients who are legionella urine antigen positive, clinicians should send respiratory specimens such as sputum and request legionella culture [D]. This is to aid outbreak and source investigation with the aim of preventing further cases.

\section{Tests for Mycoplasma pneumoniae}

33. Where available, PCR of respiratory tract samples such as sputum should be the method of choice for the diagnosis of mycoplasma pneumonia. [D]

34. In the absence of a sputum or lower respiratory tract sample, and where mycoplasma pneumonia is suspected on clinical and epidemiological grounds, a throat swab for Mycoplasma pneumoniae PCR is recommended. [D]

35. Serology with the complement fixation test and a range of other assays is widely available, although considerable caution is required in interpretation of results. [C]

\section{Tests for Chlamydophila species}

36. Chlamydophila antigen and/or PCR detection tests should be available for invasive respiratory samples from patients with high severity CAP or where there is a strong suspicion of psittacosis. [D]

37. The complement fixation test remains the most suitable and practical serological assay for routine diagnosis of respiratory Chlamydophila infections. $[\mathrm{B}-]$ There is no currently available serological test that can reliably detect acute infection due to $C$ pneumoniae.

\section{PCR and serological tests for other respiratory pathogens}

38. Where PCR for respiratory viruses and atypical pathogens is readily available or obtainable locally, this is preferred to serological investigations. [D]

39. Where available, paired serology tests can be considered for patients with high severity CAP where no particular 
microbiological diagnosis has been made by other means (eg, culture, urine antigen, PCR) and who fail to improve, and/or where there are particular epidemiological risk factors. [D] The date of onset of symptoms should be clearly indicated on all serological request forms. [D]

40. Serological tests may be extended to all patients admitted to hospital with CAP during outbreaks and when needed for the purposes of surveillance. The criteria for performing serology tests in these circumstances should be agreed locally between clinicians, laboratories and public health. [D]

\section{Severity assessment (Section 6)}

\section{What severity assessment strategy is recommended?}

41. Clinical judgement is essential in disease severity assessment. [D]

42. The stability of any comorbid illness and a patient's social circumstances should be considered when assessing disease severity. [D]

\section{Severity assessment of CAP in patients seen in the community}

43. For all patients, clinical judgement supported by the CRB65 score should be applied when deciding whether to treat at home or refer to hospital. [D]

44. Patients who have a CRB65 score of 0 are at low risk of death and do not normally require hospitalisation for clinical reasons. $[\mathrm{B}+]$

45. Patients who have a CRB65 score of 1 or 2 are at increased risk of death, particularly with a score of 2 , and hospital referral and assessment should be considered. [B+]

46. Patients who have a CRB65 score of 3 or more are at high risk of death and require urgent hospital admission. [B+]

47. When deciding on home treatment, the patient's social circumstances and wishes must be taken into account in all instances. [D]

\section{Severity assessment of CAP in patients seen in hospital}

48. For all patients, the CURB65 score should be interpreted in conjunction with clinical judgement. [D]

49. Patients who have a CURB65 score of 3 or more are at high risk of death. These patients should be reviewed by a senior physician at the earliest opportunity to refine disease severity assessment and should usually be managed as having high severity pneumonia. Patients with CURB65 scores of 4 and 5 should be assessed with specific consideration to the need for transfer to a critical care unit (high dependency unit or intensive care unit). [B+]

50. Patients who have a CURB65 score of 2 are at moderate risk of death. They should be considered for short-stay inpatient treatment or hospital-supervised outpatient treatment. $[\mathrm{B}+]$

51. Patients who have a CURB65 score of 0 or 1 are at low risk of death. These patients may be suitable for treatment at home. $[\mathrm{B}+]$

52. When deciding on home treatment, the patient's social circumstances and wishes must be taken into account in all instances. [D]

\section{Reviewing severity status after initial assessment}

53. Regular assessment of disease severity is recommended for all patients following hospital admission. The "post take" round by a senior doctor and the medical team provides one early opportunity for this review. [D]
54. All patients deemed at high risk of death on admission to hospital should be reviewed medically at least 12-hourly until shown to be improving. [D]

\section{General management (Section 7)}

General management strategy for patients treated in the community

55. Patients with suspected CAP should be advised to rest, to drink plenty of fluids and not to smoke. [D]

56. Pleuritic pain should be relieved using simple analgesia such as paracetamol. [D]

57. The need for hospital referral should be assessed using the criteria recommended in section 6 . [C]

58. Pulse oximetry, with appropriate training, should be available to general practitioners and others responsible for the assessment of patients in the out-of-hours setting for the assessment of severity and oxygen requirement in patients with CAP and other acute respiratory illnesses. [D]

\section{Review policy for patients managed in the community}

59. Review of patients in the community with CAP is recommended after $48 \mathrm{~h}$ or earlier if clinically indicated Disease severity assessment should form part of the clinical review. [D]

60. Those who fail to improve after $48 \mathrm{~h}$ of treatment should be considered for hospital admission or chest radiography. [D]

\section{General management strategy for patients treated in hospital}

61. All patients should receive appropriate oxygen therapy with monitoring of oxygen saturations and inspired oxygen concentration with the aim to maintain arterial oxygen tension $\left(\mathrm{PaO}_{2}\right)$ at $\geqslant 8 \mathrm{kPa}$ and oxygen saturation $\left(\mathrm{SpO}_{2}\right)$ 94-98\%. High concentrations of oxygen can safely be given in patients who are not at risk of hypercapnic respiratory failure. [D]

62. Oxygen therapy in patients at risk of hypercapnic respiratory failure complicated by ventilatory failure should be guided by repeated arterial blood gas measurements. [C]

63. Patients should be assessed for volume depletion and may require intravenous fluids. [C]

64. Prophylaxis of venous thromboembolism with low molecular weight heparins should be considered for all patients who are not fully mobile. $[\mathrm{A}+]$

65. Nutritional support should be given in prolonged illness. [C]

66. Medical condition permitting, patients admitted to hospital with uncomplicated CAP should sit out of bed for at least 20 min within the first $24 \mathrm{~h}$ and mobility should be increased each subsequent day of hospitalisation. [A-]

67. Patients admitted with uncomplicated pneumonia should not be treated with traditional airway clearance techniques routinely. $[\mathrm{B}+]$

68. Patients should be offered advice regarding expectoration if there is sputum present. [D]

69. Airway clearance techniques should be considered if the patient has sputum and difficulty with expectoration or in the event of a pre-existing lung condition. [D]

\section{Monitoring in hospital}

70. Temperature, respiratory rate, pulse, blood pressure, mental status, oxygen saturation and inspired oxygen 
concentration should be monitored and recorded initially at least twice daily and more frequently in those with severe pneumonia or requiring regular oxygen therapy. [C]

71. C-reactive protein should be remeasured and a chest radiograph repeated in patients who are not progressing satisfactorily after 3 days of treatment. $[\mathrm{B}+]$

72. Patients should be reviewed within 24 h of planned discharge home, and those suitable for discharge should not have more than one of the following characteristics present (unless they represent the usual baseline status for that patient): temperature $>37.8^{\circ} \mathrm{C}$, heart rate $>100 / \mathrm{min}$, respiratory rate $>24 / \mathrm{min}$, systolic blood pressure $<90 \mathrm{~mm} \mathrm{Hg}$, oxygen saturation $<90 \%$, inability to maintain oral intake and abnormal mental status. $[\mathrm{B}+]$

\section{Critical care management of CAP}

73. Patients with CAP admitted to ICUs should be managed by specialists with appropriate training in intensive care working in close collaboration with specialists in respiratory medicine. [D]

74. Neither non-invasive ventilation (NIV) nor continuous positive airways pressure (CPAP) support is routinely indicated in the management of patients with respiratory failure due to CAP. $[\mathrm{A}-]$

75. If a trial of non-invasive support is considered indicated in CAP, it must only be conducted in a critical care area where immediate expertise is available to enable a rapid transition to invasive ventilation. [D]

76. Steroids are not recommended in the routine treatment of high severity CAP. $[\mathrm{A}+]$

77. Granulocyte colony stimulating factor is not routinely recommended as an adjunct to antibiotics. $[\mathrm{A}+]$

\section{Follow-up arrangements}

78. Clinical review should be arranged for all patients at around 6 weeks, either with their general practitioner or in a hospital clinic. [D]

79. At discharge or at follow-up, patients should be offered access to information about CAP such as a patient information leaflet. [D]

80. It is the responsibility of the hospital team to arrange the follow-up plan with the patient and the general practitioner. [D]

\section{Antibiotic management (Section 8)}

Empirical antibiotic choice for adults treated in the community

81. For patients treated in the community, amoxicillin remains the preferred agent at a dose of $500 \mathrm{mg}$ three times daily. $[\mathrm{A}+]$

82. Either doxycycline [D] or clarithromycin $[\mathrm{A}-]$ are appropriate as an alternative choice, and for those patients who are hypersensitive to penicillins.

83. Those with features of moderate or high severity infection should be admitted urgently to hospital. [C]

\section{Should general practitioners administer antibiotics prior to hospital} transfer?

84. For those patients referred to hospital with suspected CAP and where the illness is considered to be life-threatening, general practitioners should administer antibiotics in the community. [D] Penicillin G $1.2 \mathrm{~g}$ intravenously or amoxicillin $1 \mathrm{~g}$ orally are the preferred agents.
85. For those patients referred to hospital with suspected high severity CAP and where there are likely to be delays of over $6 \mathrm{~h}$ in the patient being admitted and treated in hospital, general practitioners should consider administering antibiotics in the community. [D]

When should the first dose of antibiotics be given to patients admitted to hospital?

86. A diagnosis of CAP should be confirmed by chest radiography before the commencement of antibiotics in the majority of patients. Selected patients with life-threatening disease should be treated based on a presumptive clinical diagnosis of CAP. In such instances, an immediate chest radiograph to confirm the diagnosis or to indicate an alternative diagnosis is indicated. [D]

87. All patients should receive antibiotics as soon as the diagnosis of CAP is confirmed. [D] This should be before they leave the initial assessment area (emergency department or acute medical unit). The objective for any service should be to confirm a diagnosis of pneumonia with chest radiography and initiate antibiotic therapy for the majority of patients with CAP within $4 \mathrm{~h}$ of presentation to hospital. [B-]

Empirical antibiotic choice for adults hospitalised with low severity CAP

88. Most patients with low severity CAP can be adequately treated with oral antibiotics. [C]

89. Oral therapy with amoxicillin is preferred for patients with low severity CAP who require hospital admission for other reasons such as unstable comorbid illnesses or social needs. [D]

90. When oral therapy is contraindicated, recommended parenteral choices include intravenous amoxicillin or benzylpenicillin, or clarithromycin. [D]

Empirical antibiotic choice for adults hospitalised with moderate severity CAP

91. Most patients with moderate severity CAP can be adequately treated with oral antibiotics. [C]

92. Oral therapy with amoxicillin and a macrolide is preferred for patients with moderate severity CAP who require hospital admission. [D]

- Monotherapy with a macrolide may be suitable for patients who have failed to respond to an adequate course of amoxicillin before admission. Deciding on the adequacy of prior therapy is difficult and is a matter of individual clinical judgement. It is therefore recommended that combination antibiotic therapy is the preferred choice in this situation and that the decision to adopt monotherapy is reviewed on the "post take" round within the first $24 \mathrm{~h}$ of admission. [D]

93. When oral therapy is contraindicated, the preferred parenteral choices include intravenous amoxicillin or benzylpenicillin, together with clarithromycin. [D]

94. For those intolerant of penicillins or macrolides, oral doxycyline is the main alternative agent. Oral levofloxacin and oral moxifloxacin are other alternative choices. [D]

95. When oral therapy is contraindicated in those intolerant of penicillins, recommended parenteral choices include levofloxacin monotherapy or a second-generation (eg, cefuroxime) or third-generation (eg, cefotaxime or ceftriaxone) cephalosporin together with clarithromycin. [D] 
Empirical antibiotic choice for adults hospitalised with high severity CAP

96. Patients with high severity pneumonia should be treated immediately after diagnosis with parenteral antibiotics. $[\mathrm{B}-]$

97. An intravenous combination of a broad-spectrum $\beta$ lactamase stable antibiotic such as co-amoxiclav together with a macrolide such as clarithromycin is preferred. [C]

98. In patients allergic to penicillin, a second-generation (eg, cefuroxime) or third-generation (eg, cefotaxime or ceftriaxone) cephalosporin can be used instead of coamoxiclav, together with clarithromycin. [C]

When should the intravenous or the oral route be chosen?

99. The oral route is recommended in those with low and moderate severity CAP admitted to hospital provided there are no contraindications to oral therapy. $[\mathrm{B}+]$

\section{When should the intravenous route be changed to oral?}

100. Patients treated initially with parenteral antibiotics should be transferred to an oral regimen as soon as clinical improvement occurs and the temperature has been normal for $24 \mathrm{~h}$, providing there is no contraindication to the oral route. Pointers to clinical improvement are given in box $4 .[\mathrm{B}+]$

101. The choice of route of administration should be reviewed initially on the "post take" round and then daily. [D]

102. Ward pharmacists could play an important role in facilitating this review by highlighting prescription charts where parenteral antibiotic therapy continues. [D]

\section{Which oral antibiotics are recommended on completion of intravenous therapy?}

103. The antibiotic choices for the switch from intravenous to oral are straightforward where there are effective and equivalent oral and parenteral formulations. [C]

104. In the case of parenteral cephalosporins, the oral switch to co-amoxiclav $625 \mathrm{mg}$ three times daily is recommended rather than to oral cephalosporins. [D]

105. For those treated with benzylpenicillin + levofloxacin, oral levofloxacin with or without oral amoxicillin $500 \mathrm{mg}-1.0 \mathrm{~g}$ three times daily is recommended. [D]

\section{How long should antibiotics be given for?}

106. For patients managed in the community and for most patients admitted to hospital with low or moderate severity and uncomplicated pneumonia, 7 days of appropriate antibiotics is recommended. [C]

107. For those with high severity microbiologically-undefined pneumonia, 7-10 days of treatment is proposed. This may need to be extended to 14 or 21 days according to clinical judgement; for example, where Staphylococcus aureus or Gram-negative enteric bacilli pneumonia is suspected or confirmed. [C]

\section{Failure of initial empirical therapy}

108. When a change in empirical antibiotic therapy is considered necessary, a macrolide could be substituted for or added to the treatment for those with low severity pneumonia treated with amoxicillin monotherapy in the community or in hospital. [D]

109. For those with moderate severity pneumonia in hospital on combination therapy, changing to doxycycline or a fluoroquinolone with effective pneumococcal cover are alternative options. [D]

110. Adding a fluoroquinolone is an option for those with high severity pneumonia not responding to a $\beta$-lactam $/$ macrolide combination antibiotic regimen. [D]

\section{Avoiding inappropriate antibiotic prescribing}

111. The diagnosis of CAP and the decision to start antibiotics should be reviewed by a senior clinician at the earliest opportunity. There should be no barrier to discontinuing antibiotics if they are not indicated. [D]

112. The indication for antibiotics should be clearly documented in the medical notes. [D]

113. The need for intravenous antibiotics should be reviewed daily. [D]

114. De-escalation of therapy, including the switch from intravenous to oral antibiotics, should be considered as soon as is appropriate, taking into account response to treatment and changing illness severity. [D]

115. Strong consideration should be given to narrowing the spectrum of antibiotic therapy when specific pathogens are identified or when the patient's condition improves. [D]

116. Where appropriate, stop dates should be specified for antibiotic prescriptions. [D]

\section{Optimum antibiotic choices when specific pathogens have been identified}

117. If a specific pathogen has been identified, the antibiotic recommendations are as summarised in table 6. [C]

\section{Specific issues regarding the management of Legionnaires' disease}

118. As soon as a diagnosis of legionella pneumonia has been made, the clinician should liaise with the clinical microbiologist to confirm that the local Health Protection Unit has been informed. The Health Protection Unit is responsible for promptly investigating the potential sources of infection. [D]

119. The clinician should assist, where appropriate, in the gathering of clinical and epidemiological information from the patient and their relatives to aid the source investigation. [D]

120. Sputum or respiratory secretions should be sent off specifically for legionella culture in proven cases, even after appropriate antibiotics have started. [D]

121. For low and moderate severity community acquired legionella pneumonia, an oral fluoroquinolone is recommended. In the unusual case when this is not possible due to patient intolerance, a macrolide is an alternative. [D] Antibiotics are not required for the non-pneumonic selflimiting form of legionellosis-pontiac fever. [D]

122. For the management of high severity or life-threatening legionella pneumonia, a fluoroquinolone is recommended. For the first few days this can be combined with a macrolide (azithromycin is an option in countries where it is used for pneumonia) or rifampicin as an alternative. [D] Clinicians should be alert to the potential small risk of cardiac electrophysiological abnormalities with quinolonemacrolide combinations.

123. Duration of therapy should be as for microbiologicallyundefined CAP (for those with low to moderate severity pneumonia, 7 days treatment is proposed; for those with high severity pneumonia, 7-10 days treatment is 
proposed-this may need to be extended to 14 or 21 days) and should be guided by clinical judgement. [D]

Specific issues regarding Panton-Valentine Leukocidin-producing Staphylococcus aureus (PVL-SA)

124. PVL-SA infection is a rare cause of high severity pneumonia and can be associated with rapid lung cavitation and multiorgan failure. Such patients should be considered for critical care admission. [D]

125. If PVL-SA necrotising pneumonia is strongly suspected or confirmed, clinicians should liaise urgently with the microbiology department in relation to further antibiotic management and consider referral to the respiratory medicine department for clinical management advice. [D]

126. Current recommendations for the antibiotic management of strongly suspected necrotising pneumonia include the addition of a combination of intravenous linezolid $600 \mathrm{mg}$ twice daily, intravenous clindamycin $1.2 \mathrm{~g}$ four times a day and intravenous rifampicin $600 \mathrm{mg}$ twice daily to the initial empirical antibiotic regimen. As soon as PVL-SA infection is either confirmed or excluded, antibiotic therapy should be narrowed accordingly. [D]

\section{Complications and failure to improve (Section 9)}

Failure to improve in hospital

127. For patients who fail to improve as expected, there should be a careful review by an experienced clinician of the clinical history, examination, prescription chart and results of all available investigation results. [D]

128. Further investigations including a repeat chest radiograph, C-reactive protein and white cell count and further specimens for microbiological testing should be considered in the light of any new information after the clinical review. [D]

129. Referral to a respiratory physician should be considered. [D]

\section{Common complications of CAP}

130. Early thoracocentesis is indicated for all patients with a parapneumonic effusion. [D]

131. Those found to have an empyema or clear pleural fluid with $\mathrm{pH}<7.2$ should have early and effective pleural fluid drainage. [C]

132. The British Thoracic Society guidelines for the management of pleural infection should be followed. [D]

133. Less usual respiratory pathogens including anaerobes, $S$ aureus, Gram-negative enteric bacilli and $S$ milleri should be considered in the presence of lung abscess. [D]

134. Prolonged antibiotic therapy of up to 6 weeks depending on clinical response and occasionally surgical drainage should be considered. [D]

\section{Prevention and vaccination (Section 10)}

Influenza and pneumococcal vaccination

135. Department of Health guidelines in relation to influenza and pneumococcal immunisation of at-risk individuals should be followed. [C]

136. All patients aged $>65$ years or at risk of invasive pneumococcal disease who are admitted with CAP and who have not previously received pneumococcal vaccine should receive 23 -valent pneumococcal polysaccharide vaccine (23-PPV) at convalescence in line with the Department of Health guidelines. [C]

\section{Smoking cessation}

137. Smoking cessation advice should be offered to all patients with CAP who are current smokers according to smoking cessation guidelines issued by the Health Education Authority. $[\mathrm{B}+]$

\section{SECTION 1 INTRODUCTION \\ 1.1 Scope of these guidelines}

- These guidelines refer to the management of adults with community acquired pneumonia (CAP) of all ages in the community or in hospital. They have been developed to apply to the UK healthcare system and population. They might equally be applicable to any other countries which operate similar healthcare services (figs 1 and 2).

- They are NOT aimed at patients with known predisposing conditions such as cancer or immunosuppression admitted with pneumonia to specialist units such as oncology, haematology, palliative care, infectious diseases units or AIDS units.

- They do NOT apply to the much larger group of adults with non-pneumonic lower respiratory tract infection, including illnesses labelled as acute bronchitis, acute exacerbations of chronic obstructive pulmonary disease or "chest infections".

\subsection{Introduction}

The British Thoracic Society (BTS) guidelines for the management of Community Acquired Pneumonia (CAP) in Adults were published in December $2001^{1}$ and superseded guidelines published in 1993. A web-based update of the 2001 guidelines was published in 2004. ${ }^{2}$ The 2004 guidelines assessed relevant evidence published up to August 2003.

This update represents a further assessment of published or available evidence from August 2003 to August 2008. An identical search strategy, assessment of relevance and appraisal of articles and grading system was used (see Section 1.8 and Appendices 1-4)

- This document incorporates material from the 2001 and 2004 guidelines and supersedes the previous guideline documents.

\subsection{Definitions}

1.3.1 Defining community acquired pneumonia (CAP)

The diagnosis in hospital will be made with the benefit of a chest radiograph. In the community, the recognition and definition of CAP by general practitioners in the UK, without the benefit of investigations or radiology, poses greater challenges and the diagnosis will often be based only on clinical features.

\subsubsection{Defining CAP in a community setting}

The clinical definition of CAP that has been used in community studies has varied widely but has generally included a complex of symptoms and signs both from the respiratory tract and regarding the general health of the patient. Features such as fever $\left(>38^{\circ} \mathrm{C}\right)$, pleural pain, dyspnoea and tachypnoea and signs on physical examination of the chest (particularly when new and localising) seem most useful when compared with the gold standard of radiological diagnosis of CAP. ${ }^{\text {[II] }}$ See Section 5.1 for a fuller discussion pertaining to the clinical diagnosis of CAP managed in the community. 
Figure 1 Synopsis of the management of adult patients seen in the community with suspected community acquired pneumonia, with cross reference to relevant sections in the document text.

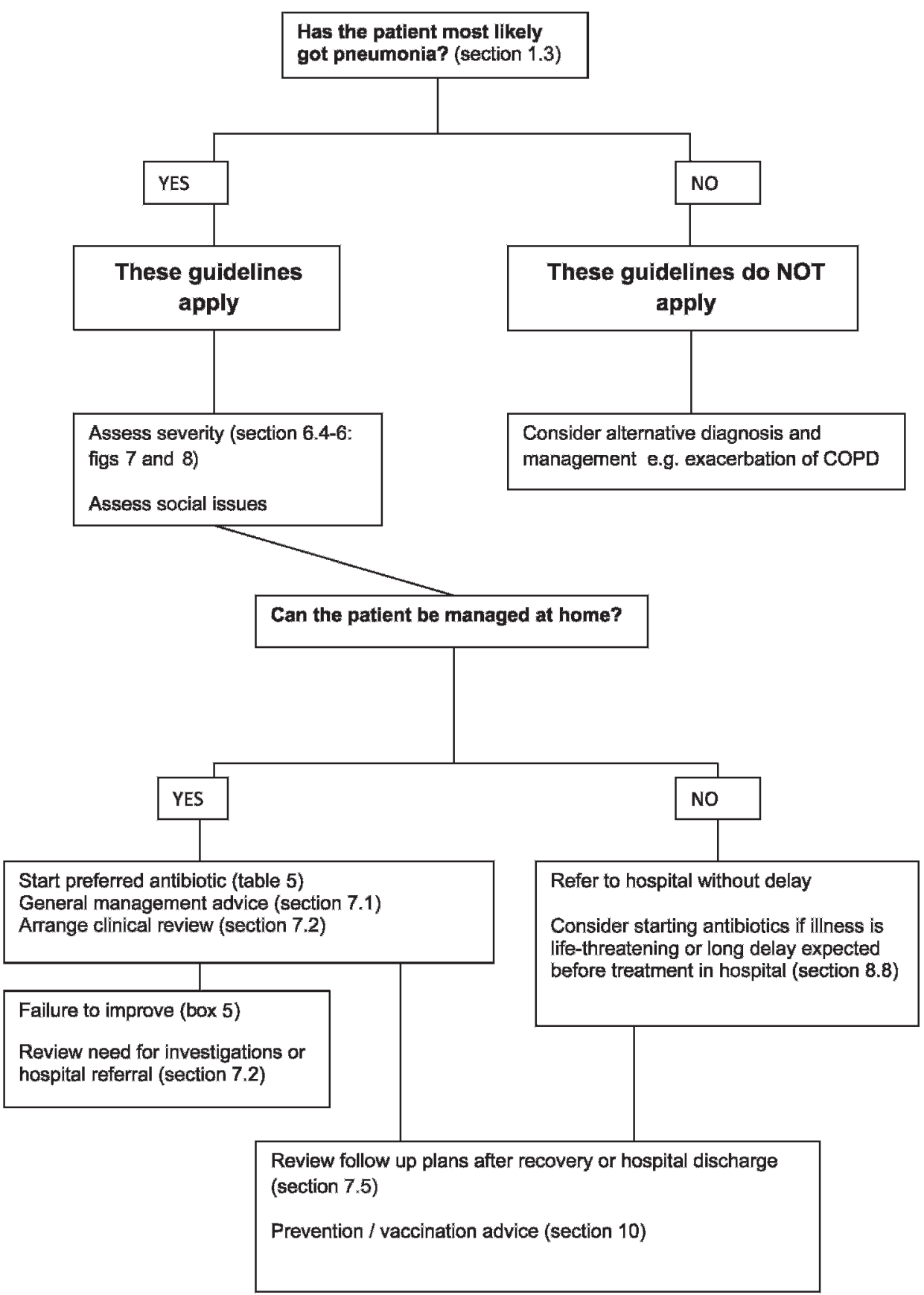

For the purposes of these guidelines, CAP in the community has been defined as:

- Symptoms of an acute lower respiratory tract illness (cough and at least one other lower respiratory tract symptom).

- New focal chest signs on examination.

- At least one systemic feature (either a symptom complex of sweating, fevers, shivers, aches and pains and/or temperature of $38^{\circ} \mathrm{C}$ or more).

- No other explanation for the illness, which is treated as CAP with antibiotics.

\subsubsection{Definition of CAP in patients admitted to hospital (when a} chest radiograph is available)

Studies of CAP from different countries have used very different definitions and inclusion criteria; $;^{3-5}$ most have required a combination of symptoms, signs and radiological features. The BTS study of CAP used a definition which included: an acute illness with radiographic shadowing which was at least segmental or present in more than one lobe and was not known to be previously present or due to other causes. ${ }^{6}$ Like most studies, cases were excluded if pneumonia occurred distal to a known carcinoma or foreign body.

For the purposes of these guidelines, CAP in hospital has been defined as:

- Symptoms and signs consistent with an acute lower respiratory tract infection associated with new radiographic shadowing for which there is no other explanation (eg, not pulmonary oedema or infarction).

- The illness is the primary reason for hospital admission and is managed as pneumonia. 
Figure 2 Synopsis of the management of adult patients seen in hospital with suspected community acquired pneumonia, with cross reference to relevant sections in the document text.

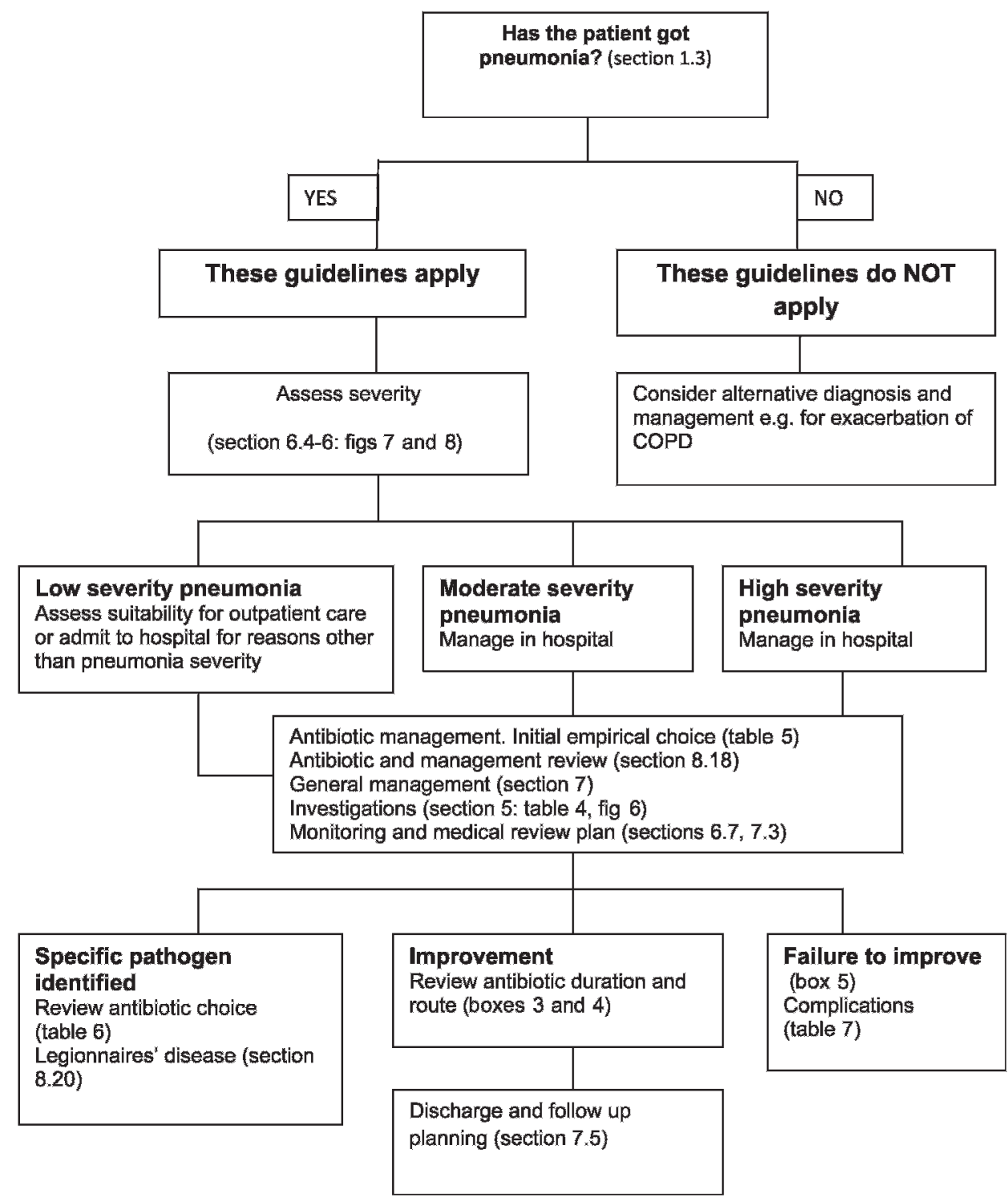

1.3.2 Defining the terms "atypical pneumonia" and "atypical pathogens"

The term "atypical pneumonia" has outgrown its historical usefulness and we do not recommend its continued use as it implies (incorrectly) a distinctive clinical pattern (see Section 4.2).

For the purposes of these guidelines, the term "atypical pathogens" is used to define infections caused by:

- Mycoplasma pneumoniae;

- Chlamydophila pneumoniae;

- Chlamydophila psittaci; and

- Coxiella burnetii.

These pathogens are characterised by being difficult to diagnose early in the illness and are sensitive to antibiotics other than $\beta$-lactams such as macrolides, tetracyclines or fluoroquinolones which are concentrated intracellularly, which is the usual site of replication of these pathogens. As such, we conclude that the term "atypical pathogens" is still useful to clinicians in guiding discussion about aetiology and management of CAP.
Legionella spp, although sharing some of these characteristics, are not considered to be an "atypical pathogen" for the purpose of this document as there are different species and these can be acquired both in the community and hospital environment.

\subsubsection{Defining the term "elderly"}

There is no agreed age cut-off to define the term "elderly". When referring to published research, wherever possible we define the age limits used in the relevant studies.

\subsection{What is the target end user audience?}

We want these guidelines to be of value to:

- Hospital-based medical and other staff involved with managing adult patients with CAP.

- General practitioners.

- Those teaching about the subject at both undergraduate and postgraduate level.

The guidelines have been developed to apply to the UK healthcare system and population, but they might also be of value to other countries which operate similar healthcare 
services, with appropriate modification to take into account differences in licensing and availability of antimicrobial agents.

\subsection{What patient populations are we including and excluding?}

These guidelines address the management of unselected adults with CAP who are managed by their general practitioner or admitted to hospital as an emergency.

Although there are similarities in the principles of management between pneumonic lower respiratory tract infection (ie, CAP) and non-pneumonic lower respiratory tract infection, there are differences in the aetiology, severity assessment, management and outcome. Recommendations for the antibiotic management of acute exacerbations of chronic obstructive pulmonary disease (COPD) are included in the guideline on the management of COPD published by the National Institute of Health and Clinical Excellence (NICE). ${ }^{\text {? }}$

We do not consider the management of pneumonia in:

- Patients where the pneumonia is an expected terminal event or who are known to have lung cancer, pulmonary tuberculosis or cystic fibrosis or primary immune deficiency or secondary immune deficiency related to HIV infection, or drug or systemic disease-induced immunosuppression. We do include patients receiving oral corticosteroid therapy as this is a not uncommon situation for patients admitted on medical take.

- Patients who have been in hospital within the previous 10 days and may have hospital acquired pneumonia. Patients admitted from healthcare facilities such as nursing homes and residential homes will be commented on separately.

- Children with CAP (please refer to the BTS guidelines for the management of CAP in $\operatorname{childhood}^{8}$ ).

\subsection{What changes have happened in the area of CAP since the 2004 guidelines?}

- Concerns regarding health care-associated infections (HCAIs), particularly methicillin-resistant Staphylococcus aureus (MRSA) and Clostridium difficile infection, have grown in recent years. These HCAIs are associated with volume of antibiotic use. Antibiotic stewardship should now be an essential responsibility for all clinicians. Measures to avoid and reduce inappropriate antibiotic use are therefore at the forefront of management strategies for all infective episodes. ${ }^{9}$

- Fluoroquinolone antibiotics with enhanced activity against Gram-positive organisms (the so-called "respiratory quinolones" such as levofloxacin and moxifloxacin) have been widely available for some years now. Their activity against most major respiratory pathogens led initially to widespread use of these antibiotics for respiratory tract infections, including CAP. However, more recently these antibiotics have been associated with both methicillin-resistant $S$ aureus (MRSA) and $C$ difficile infections. ${ }^{10}{ }^{11}$ This has promoted increasing pressure to limit the use of these antibiotics in favour of other classes of antibiotics where appropriate. ${ }^{9}$

- Antimicrobial resistance in Streptococcus pneumoniae was noted to rise in the late 1990s. Fortunately, a reversal of this trend has been observed in the last 5 years, with rates of penicillin-resistant $S$ pneumoniae in the UK remaining below 4\% (see Section 8.4).

- Pneumonia admissions to hospital rose by $34 \%$ between 1997-8 and 2004-5. ${ }^{12}$ This was matched by an increase in admissions to intensive care units for $\mathrm{CAP}^{13}$ (see Section 2.1).
- The processes for managing acutely ill medical patients admitted to hospital have undergone important changes. The specialty of acute medicine has developed substantially and, in many hospitals, teams run by acute medicine physicians are already taking responsibility for the early stages of acutely ill medical patients. This shift, together with the demands on junior doctors' hours arising from the European Working Time Directive, mean that patient care increasingly involves different teams of doctors. Effective handover between teams, careful patient review and coherent clinical guidelines are key aspects of patient management in this context. ${ }^{14}$

- Timeliness of treatment has enlarged as a priority in clinical care processes. This is perhaps most evident in the "4-hour admission to treatment" target applied to emergency departments across the UK. ${ }^{15}$ Increased attention to speed to treatment as a measure of performance may have the inadvertent effect of increasing the inappropriate or excessive use of antibiotics in patients with suspected but unconfirmed CAP, thus exacerbating any existing problems with HCAIs (see Section 8.9).

- Newer microbiological tests for the detection of infection by respiratory pathogens such as urine antigen tests are becoming increasing available routinely, while previously established tests such as complement fixation tests are gradually being phased out.

\subsection{Guidelines Committee membership}

The Guidelines Committee was established in January 2008 with representatives from a range of professional groups including the Royal College of General Practitioners, Royal College of Physicians, British Geriatric Society, British Infection Society, British Society for Antimicrobial Chemotherapy, General Practice Airways Group, Health Protection Agency and the Society for Acute Medicine (see Section 11). Three members in the current committee also served on the 2001 and 2004 Guidelines Committee.

The Guidelines Committee agreed the remit of the guidelines. The Centre for Reviews and Dissemination and Centre for Health Economics at the University of York was commissioned by the BTS to undertake literature searches on behalf of the Guidelines Committee.

\subsection{How the evidence was assimilated into the guidelines}

\subsubsection{Literature searches}

Systematic electronic database searches were conducted in order to identify potentially relevant studies for inclusion in the CAP guidelines. For each topic area the following databases were searched: Ovid MEDLINE (including MEDLINE In Process), Ovid EMBASE, Ovid CINAHL and the Cochrane Library (including the Cochrane Database of Systematic Reviews, the Database of Abstracts of Reviews of Effects, the Cochrane Central Register of Controlled Trials, the Health Technology Assessment database and the NHS Economic Evaluation Database).

The searches were first run in December 2007 and were updated in August 2008. Searches included a combination of indexing terms and free text terms, and were limited to English language publications only. Full search strategies for each database are available in the web-based supplement.

\subsubsection{Appraisal of the literature}

One individual (HR) read the title and abstract of each article retrieved by the literature searches and decided whether the 
paper was definitely relevant, possibly relevant or not relevant to the project. For each unique paper in the first and second category, the full paper was ordered and allocated to the relevant section(s).

The initial searches (2003-7) identified 7449 reference abstracts, of which 1603 were definitely or possibly relevant after the first screen. These were divided into groups as follows: aspiration/institutional pneumonia (141); C difficile infection related (66); pneumonia on critical care (161); aetiology (154); antibiotic therapy (420); clinical features (46); community investigations and management (68); complications and failure to improve (37); general investigations and management (288); incidence and epidemiology (55); microbiology investigations (86); prevention (232); radiology (15); severity assessment (134).

The second search (2007-8) identified 1143 reference abstracts, of which only 177 were definitely or possibly relevant. These were divided into the same groups: aspiration/institutional pneumonia (11); $C$ difficile infection related (5); pneumonia on critical care (10); aetiology (22); antibiotic therapy (36); clinical features (0); community investigations and management (3); complications and failure to improve; ${ }^{16}$ general investigations and management (20); incidence and epidemiology (8); microbiology investigations (10); prevention (9); radiology (2); severity assessment (26).

A total of 547 papers were retrieved and circulated for critical appraisal. The leads for each section independently judged the clinical relevance and scientific rigour of each paper assigned to them using generic study appraisal checklists (see Appendices 1 and 2) adapted from published checklists. ${ }^{17-20}$ The reliability of the evidence in each study was graded from Ia to IVb using a generic list of evidence levels (see Appendix 3) developed from existing insights and checklists. ${ }^{21} 22$ Disagreements were resolved by discussion with the section partner (see Section 11.2). Where relevant, individual references used in this document are followed by an indication of the evidence level in square brackets.

Section leads individually assessed the literature selected and wrote a short document describing study findings and related recommendations. These documents were discussed by the whole committee.

\subsubsection{Drafting of the guidelines}

The Guidelines Committee corresponded by email on a regular basis throughout the duration of the guideline development. Meetings of the full group were held in February 2008, July 2008 and November 2008. Each section lead edited the corresponding section in the 2001 guidelines document, incorporating all relevant literature and recommendations from the 2004 update and the current update. In December 2008 the guidelines were discussed at an open plenary session at the BTS Winter Conference. A revised draft guidelines document was circulated to professional bodies for endorsement in January 2009 and to the BTS Standards of Care Committee in March 2009.

\subsection{Grading of recommendations}

Recommendations were graded from $\mathrm{A}+$ to $\mathrm{D}$ (table 1) as indicated by the strength of the evidence as listed in the table in Appendix 4.

\subsection{Plans for updating these guidelines}

Following the BTS protocol for guidelines revisions, the Committee will meet on an annual basis and review new published evidence obtained from a structured literature search,
Table 1 Brief description of the generic levels of evidence and guideline statement grades used

\begin{tabular}{lll}
\hline $\begin{array}{l}\text { Evidence } \\
\text { level }\end{array}$ & Definition & $\begin{array}{l}\text { Guideline } \\
\text { statement grade }\end{array}$ \\
\hline Ia & $\begin{array}{l}\text { A good recent systematic review of studies } \\
\text { designed to answer the question of interest }\end{array}$ & A+ \\
Ib & $\begin{array}{l}\text { One or more rigorous studies designed to answer } \\
\text { the question, but not formally combined }\end{array}$ & $\mathrm{A}-$ \\
$\mathrm{II}$ & $\begin{array}{l}\text { One or more prospective clinical studies which } \\
\text { illuminate, but do not rigorously answer, the } \\
\text { question }\end{array}$ & B+ \\
III & $\begin{array}{l}\text { One or more retrospective clinical studies which } \\
\text { illuminate, but do not rigorously answer, the }\end{array}$ & B- \\
IVa & $\begin{array}{l}\text { question } \\
\text { Formal combination of expert views }\end{array}$ & C \\
IVb & Other information & D \\
\hline A
\end{tabular}

A fuller description is given in Section 1 and Appendices 1-4.

comment on any newly licensed and relevant antibiotics and issue guideline updates or revisions as necessary. Important changes will be posted on the BTS website (www.brit-thoracic. org.uk). The membership of the Guideline Committee will change over time on a rolling programme dictated by the BTS Standards of Care Committee policy for the Guideline Committee membership.

\subsection{Implementation of the guidelines}

We expect that these guidelines will act as a framework for local development or modification of protocols after discussion with local clinicians and management. The subsequent dissemination, implementation and evaluation of these guidelines should be undertaken by the hospital Quality and Clinical Effectiveness Group in conjunction with relevant committees such as those responsible for therapeutics, antibiotic prescribing or protocol development. Countries with similar health service systems will also find the framework of value, adapting the guidelines to take into account any relevant national differences in disease presentation and the availability of investigations and antimicrobial agents.

\subsection{Auditing CAP management}

The management of CAP is a sufficiently common and important issue to warrant the development of audit measures of the process of care and outcome to evaluate the quality of care for CAP, using guidelines as a standard of management.

An audit tool has been developed and is available through the BTS website (www.brit-thoracic.org.uk).

\section{SECTION 2 INCIDENCE, MORTALITY AND ECONOMIC CONSEQUENCES}

2.1 How common is adult CAP in the community and in hospital? Prospective population studies from the UK, ${ }^{23}[\mathrm{II}]$ Finland $^{24}[\mathrm{Ib}]$ and North America ${ }^{25}{ }^{[\mathrm{Ib}]}$ have reported an annual incidence of CAP diagnosed in the community of between 5 and 11 per thousand adult population. Pneumonia, diagnosed clinically by general practitioners, accounts for only $5 \%^{23}[\mathrm{lb}]$ to $12 \%{ }^{26}[\mathrm{Ib}]$ of all cases of adult lower respiratory tract infection treated with antibiotics by general practitioners in the community in the UK.

The incidence varies markedly with age, being much higher in the very young and the elderly. In a Finnish study the annual incidence in the 16-59 age group was 6 per 1000 population, for those aged $\geqslant 60$ years and 34 per 1000 population for those aged $\geqslant 75$ years. ${ }^{24}[\mathrm{Ib}]$ A similar pattern was reported from Seattle, $\mathrm{USA}^{25}{ }^{[\mathrm{Ib}]}$ 
Population-based studies of the incidence of CAP requiring hospitalisation have reported overall incidences of 1.1 per 1000 adult population per annum in Canada, ${ }^{27}\left[{ }^{[\mathrm{bb}]} 2.6\right.$ per 1000 in Spain, ${ }^{6}{ }^{[\mathrm{III}} 2.7$ per 1000 population in Ohio, USA ${ }^{6}[\mathrm{[b]}]$ and 4 per 1000 population in Pennsylvanian hospitals, USA. ${ }^{28}$ [III] Increasing age was associated with an increasing incidence of admission to hospital with CAP in Canada; from 1.29 per 1000 persons aged 18-39 years, to 1.91 per 1000 persons aged 4054 years, to 13.21 per 1000 persons aged 55 years or above. ${ }^{29}$ [III] A study of Hospital Episode Statistics for England between 1997 and 2005 showed a rise in hospital admissions for pneumonia over time. The age-standardised incidence of hospitalisations with a primary diagnosis of pneumonia increased by $34 \%$ between 1997-8 and 2004-5, from 1.48 to 1.98 per 1000 population. This increase was more marked in older adults. ${ }^{12}$ [III]

The proportion of adults with CAP who require hospital admission in the UK has been reported as between $22 \%{ }^{23}[$ [b] and $42 \% .{ }^{30}$ [III] This figure varies in other countries, probably dependent on the structure of the primary and secondary healthcare system. In a Finnish prospective longitudinal population study, $42 \%$ were admitted to hospital. ${ }^{24}[\mathrm{Ib}]$ A $50 \%$ admission rate was reported in one study from Spain, but this only included patients referred by their general practitioner to the hospital emergency service for confirmation of the diagnosis of CAP..$^{10[\mathrm{II}]}$

In Seattle, USA 15\% were hospitalised. ${ }^{31}[$ [b] In the Pneumonia Patient Outcomes Research multicentre prospective cohort study of CAP in America, $41 \%$ of adults studied were managed initially as outpatients and the remainder were admitted to hospital. Of those initially treated as outpatients, only $7.5 \%$ were subsequently admitted, $56 \%$ because of the CAP and the rest because of worsening of a comorbid illness. ${ }^{32}$ [Ib]

The proportion of adults hospitalised with CAP who require management on an intensive care unit (ICU) varies from $1.2 \%$ in one Spanish study ${ }^{12}\left[\mathrm{III}\right.$ and $5 \%$ in the BTS multicentre study ${ }^{65}$ [II] to $10 \%$ in another Spanish study. ${ }^{33}$ [II] Previously, between $8 \%{ }^{13}[\mathrm{III}]$ and $10 \%{ }^{34}$ [III] of medical admissions to an ICU were found to be for severe CAP. An analysis of admissions to ICUs across England, Wales and Northern Ireland between 1995 and 2004 found CAP to be the cause of $5.9 \%$ of all ICU admissions. There was an increase in CAP requiring intensive care from 12.8 per unit in 1996 to 29.2 per unit in 2004. This represented an increase of $128 \%$ compared with a rise in the total number of admissions to ICUs of only $24 \% .^{13}$ [III]

\section{Summary}

- The annual incidence in the community is $5-11$ per 1000 adult population. [Ib]

- CAP accounts for $5-12 \%$ of all cases of adult lower respiratory tract infection managed by general practitioners in the community. [Ib]

- The incidence varies markedly with age, being much higher in the very young and the elderly. [Ib]

- Between $22 \%$ and $42 \%$ of adults with CAP are admitted to hospital. [Ib]

- The incidence for patients requiring admission to hospital varies with age from 1.29 per 1000 persons aged 18-39 years up to 13.21 per 1000 persons aged $\geqslant 55$ years. [III]

- The age-standardised incidence of admission to hospital increased by 34\% from 1.48 to 1.98 per 1000 population between 1997-8 and 2004-5 in England.

- Between $1.2 \%$ and $10 \%$ of adults admitted to hospital with CAP are managed on an ICU. [II]

\subsection{What is the mortality of CAP?}

The reported mortality of adults with CAP managed in the community is low and less than $1 \% .{ }^{15} 2323$ [II] [bb] [bb] Deaths in the community due to CAP are rare in the UK. In one study only seven cases were identified by coroners' post mortems over 1 year in Nottingham, a large urban city of three quarters of a million, giving an incidence of 1 per $100000 .^{23}$ [III]

The reported mortality of adults hospitalised with CAP has varied widely. The BTS multicentre study reported a mortality of $5.7 \%,{ }^{6}[\mathrm{II}]$ but did not study patients over the age of 74 years. Other UK studies have reported mortalities of $8 \%{ }^{35}[\mathrm{III}] 12 \%{ }^{36}[\mathrm{Ib}]$ and $14 \%{ }^{37}{ }^{[\mathrm{bb}]}$ Countries with similar healthcare systems have reported hospital mortality rates of $4 \%{ }^{24}[\mathrm{Ib}] 7 \%,{ }^{38}[\mathrm{III}] 8 \%{ }^{241}[\mathrm{[b}]$ and $10 \% .^{39}[\mathrm{Ib}]$

The longer term mortality of CAP is high, reflecting the frailty of many patients who develop CAP in the first instance. In a US study the 90-day all-cause mortality was $8.7 \%$ and mortality at 5.9 years was $39.1 \%$. Age, level of education, male sex and nursing home residence were independently associated with long-term mortality. ${ }^{40}$ [II] Other studies found long-term mortality to be $20.8 \%$ at 1 year, $34.1 \%$ at a mean of 901 days and $35.8 \%$ at 5 years. ${ }^{41}[\mathrm{III}]$

The mortality of patients with severe CAP requiring admission to an ICU is high. This is likely to be particularly evident in health services such as the National Health Service where ICU beds are at a premium, such that only critically ill patients in need of assisted ventilation can be admitted. ICUbased studies in the UK have reported mortalities of over $50 \%, 25344243$ [III] [III] [III] [III] although a more recent analysis of admissions to ICUs across England, Wales and Northern Ireland between 1995 and 2004 reported a mortality of 34.9\% for patients with CAP. ${ }^{13}{ }^{[\mathrm{III}]}$ Nearly all of the patients required assisted ventilation. By contrast, the mortality rate in a large multicentre study of severe CAP in four French ICUs reported a mortality of $35 \%$ with a ventilation rate of only $52 \% .{ }^{25}$ [[b] Similar figures were reported from another ICU-based study in France. ${ }^{44}$ [II] In a specialist ICU in Spain, a mortality of $22 \%$ was reported, rising to $36 \%$ in the $61 \%$ of patients who required assisted ventilation. ${ }^{33}[\mathrm{II}]$

\section{Summary}

- The reported mortality of adults with CAP managed in the community in the UK is very low and less than 1\%. [Ib]

- The reported mortality of adults hospitalised with CAP in the UK has varied between $5.7 \%$ and $14 \%$. [Ib]

- The mortality of patients with severe CAP requiring admission to an ICU in the UK is high at over 30\%. [III]

- The long-term mortality of CAP is between $35.8 \%$ and $39.1 \%$ at 5 years. [II]

\subsection{What are the economic consequences of CAP?}

A prevalence-based burden of illness study estimated that CAP in the UK incurred a direct healthcare cost of $£ 441$ million annually at $1992-3$ prices. The average cost for managing pneumonia in the community was estimated at $£ 100$ per episode compared with £1700-5100 when the patient required admission to hospital. Hospitalisation accounted for $87 \%$ of the total annual cost..$^{30}$ [III]

A similar exercise conducted in 1997 in the USA calculated that annual costs of CAP amounted to $\$ 8.4$ billion, $52 \%$ of the costs being for the inpatient care for 1.1 million patients and the remaining costs for the 4.4 million outpatient consultations. The average hospital length of stay varied between 5.8 days for 
those under 65 years of age and 7.8 days for older patients. ${ }^{45}$ [III] A prospective study of costs and outcome of CAP from five hospitals in North America concluded that costs of antibiotic therapy varied widely but had no effect on outcome or mortality. Patients treated in the hospitals with the lowest costs did not have worse medical outcomes. ${ }^{46}[\mathrm{Ib}]$

\section{Summary}

- The direct costs associated with CAP are high and mostly associated with inpatient care costs. [III]

- Substantial costs savings could likely be made by strategies to prevent CAP, to reduce the requirement for hospital admission and to shorten the length of hospital stay. [III]

\subsection{What comments can be made about cost effectiveness of different therapies?}

We are not able to provide any structured guidance on this subject. Modern guidelines should attempt to provide information, not only on clinical management but also on the assessment of robust published data on cost effectiveness of therapies. However, it was noted that there is a clear deficiency of good quality comparative clinical data which would allow meaningful comparisons of management and antibiotic strategies for CAP, whether assessing for clinical or cost effectiveness outcome.

\section{Summary}

- We have not attempted a systematic appraisal of current pharmacoeconomic evidence for CAP and do not give a structured view on cost effectiveness.

- Cost effectiveness data pertinent to UK practice does not exist at the time of writing and is an area for further research.

\section{SECTION 3 AETIOLOGY AND EPIDEMIOLOGY}

\subsection{Introduction}

No two studies of the aetiology of CAP are the same. Apparent differences in the observed frequency of pathogens, while possibly real, may also be due to a number of other factors including healthcare delivery (distribution of management between primary and secondary care, hospital and ICU admission practices), population factors (such as age mix, the frequency of alcoholism, comorbid diseases, immune suppression and malignancy) and study factors (type and number of samples collected, investigations performed, result interpretation). Frequently, such details are not explicitly stated in the study methodology and, although we have not included studies which do not comply with certain standards, apparently similar studies may hide very different methodology. With the exception of elderly subjects, few adequately powered studies using the same methodology have been used to compare different population groups. Conclusions about observed differences in the following data must therefore be treated with caution.

Many of the statements in the following text arise from a comparison of studies, rather than data from individual studies that have set out to answer that question. For this reason, evidence grades follow statements to justify that conclusion, as well as individual references.

\subsection{What are the causes of adult CAP in the UK?}

These are set out in table 2, together with details of the relevant references (and grading of evidence from those individual references), grouped together by where patients have been managed-be it in the community, in hospital or on an ICU. For all these groups, a common range of pathogens is regularly identified as causes of CAP. [Ib] Although a single pathogen is identified in $85 \%$ of patients where an aetiology is found, the true frequency of polymicrobial CAP is not known and observed figures are dependent on the intensity of investigation. $S$ pneumoniae is the most frequently identified pathogen. [Ib] The relative frequency of pathogens in patients managed in the community and in hospital is probably similar, but the absence of more than one study in the community makes further conclusions uncertain. Legionella species and $S$ aureus are identified more frequently in patients managed on the ICU. [Ib] The apparent difference in the frequency of Mycoplasma pneumoniae may depend on whether or not a study is performed in an epidemic year. [II] Gram-negative enteric bacilli, Chlamydophila psittaci and Coxiella burnetii are uncommon causes of CAP. [Ib]

Since 2001 only one additional study of adults admitted to hospital with CAP has been published, ${ }^{47}$ which found a similar distribution of common causative pathogens to that in previous studies.

\subsection{What are the causes of adult CAP in similar populations elsewhere in the world?}

The results and references of relevant studies from the remainder of Europe, Australia and New Zealand and North America were compared in the earlier BTS guidelines. ${ }^{1}$ Other European studies confirm previous knowledge. ${ }^{30} 3148[\mathrm{Ib}][\mathrm{Ib}][\mathrm{II}]$ For patients managed in the community and in hospital, the frequency of pathogens is broadly similar to that in the UK. [II] This suggests that aspects of these guidelines will be applicable to other countries as well as the UK. The absence of studies using sensitive methods for pneumococcal polysaccharide capsular antigen detection for the identification of $S$ pneumoniae may be the explanation for the lower frequency outside the UK. The apparent differences in $M$ pneumoniae may relate to the presence or absence of epidemics at the time of the study. Chlamydophila pneumoniae is identified frequently in some European countries, but recent studies in Germany ${ }^{31}[\mathrm{lb}]$ and the Netherlands ${ }^{31}[\mathrm{II}]$ found frequencies of only $0.9 \%$ and $3 \%$, respectively.

Antibiotic-resistant $S$ pneumoniae appears to be no more frequent in severely ill patients admitted to the ICU than in those managed on an ordinary hospital ward in a country where such resistance is common. ${ }^{49}[\mathrm{Ib}]$ Studies of patients with severe CAP from Europe suggest a lower frequency of legionella and a higher frequency of Gram-negative enteric bacilli infections compared with the UK. These may be real or methodological. [IVa]

A frequency of $8 \%$ for non-pneumophila legionella species was found in one Dutch study. ${ }^{32}$ [II] A study of hospitalised patients in Spain ${ }^{50}[\mathrm{bb}]$ found a frequency of mixed aetiology of $13 \%$, similar to the average figure of $11 \%$ for the UK. Another Spanish study found a frequency of $5.7 \% .^{51}[\mathrm{lb}]$ A recent publication showed a high frequency of $C$ burnetii infection in the Canary Islands. ${ }^{52}[\mathrm{Ib}]$

3.4 How does the aetiology differ in certain geographical areas? Specific studies suggest a higher frequency of certain pathogens in some geographical areas as described in the 2001 BTS guidelines (table 3). ${ }^{1}$ A global study found a frequency of atypical pathogens of $20-28 \%$ of cases in different regions of the 
Table 2 Studies of community acquired pneumonia (CAP) conducted in the UK

\begin{tabular}{|c|c|c|c|}
\hline & \multicolumn{3}{|l|}{ Where managed } \\
\hline & Community & Hospital & Intensive care unit \\
\hline & 1 study* $(n=236)$ & 5 studies $\uparrow(n=1137)$ & 4 studies: $(n=185)$ \\
\hline Streptococcus pneumoniae & $36.0(29.9$ to 42.1$)$ & 39 (36.1 to 41.8$)$ & 21.6 (15.9 to 28.3 ) \\
\hline Haemophilus influenzae & $10.2(6.3$ to 14.0$)$ & $5.2(4.0$ to 6.6$)$ & 3.8 (1.5 to 7.6$)$ \\
\hline Legionella spp & $0.4(0.01$ to 2.3$)$ & 3.6 (2.6 to 4.9 ) & $17.8(12.6$ to 24.1$)$ \\
\hline Staphylococcus aureus & $0.8(0.1$ to 3.0$)$ & $1.9(1.2$ to 2.9$)$ & $8.7(5.0$ to 13.7$)$ \\
\hline Moraxella catarrhalis & $?$ & $1.9(0.6$ to 4.3$)$ & $?$ \\
\hline Gram-negative enteric bacilli & $1.3(0.3$ to 3.7$)$ & $1.0(0.5$ to 1.7$)$ & $1.6(0.3$ to 4.7$)$ \\
\hline Mycoplasma pneumoniae & $1.3(0.3$ to 3.7$)$ & $10.8(9.0$ to 12.6$)$ & $2.7(0.9$ to 6.2$)$ \\
\hline Chlamydophila pneumoniae & $?(?)$ & $13.1(9.1$ to 17.2$)$ & $?(?)$ \\
\hline Chlamydophila psittaci & $1.3(0.3$ to 3.7$)$ & 2.6 (1.7 to 3.6$)$ & $2.2(0.6$ to 5.4$)$ \\
\hline Coxiella burnetii & $0(0$ to 1.6$)$ & $1.2(0.7$ to 2.1$)$ & $0(0$ to 2.0$)$ \\
\hline All viruses & 13.1 (8.8 to 17.4$)$ & $12.8(10.8$ to 14.7$)$ & 9.7 (5.9 to 14.9$)$ \\
\hline Influenza $A$ and $B$ & $8.1(4.9$ to 12.3$)$ & $10.7(8.9$ to 12.5$)$ & $5.4(2.6$ to 9.7$)$ \\
\hline Mixed & $11.0(7.0$ to 15.0$)$ & $14.2(12.2$ to 16.3$)$ & $6.0(3.0$ to 10.4$)$ \\
\hline Other & $1.7(0.5$ to 4.3$)$ & $2(1.3$ to 3$)$ & $4.9(2.3$ to 9.0$)$ \\
\hline None & 45.3 (39.0 to 51.7$)$ & 30.8 (28.1 to 33.5$)$ & 32.4 (25.7 to 39.7$)$ \\
\hline
\end{tabular}

world. ${ }^{53}{ }^{[I I]}$ A similar figure of $23.5 \%$ was found in a multicentre South Asian study. ${ }^{54}[\mathrm{Ib}]$

Studies from Chile ${ }^{55}[\mathrm{Ib}]$ and Nicaragua ${ }^{56}[\mathrm{lb}]$ report a similar pathogen spectrum to previous European studies.

Evidence of legionella infection was found in $31.7 \%$ of nonconsecutive pneumonia cases in Trinidad ${ }^{57}{ }^{[\mathrm{II}]}$ and $5.1 \%$ of 645 consecutive cases in Brazil. ${ }^{58}[\mathrm{Ib}]$ An incidence of $5.2 \%$ for $C$ pneumoniae was found by the same group, ${ }^{58}{ }^{[11]}$ with a frequency of $8.1 \%$ being found in a Canadian study. ${ }^{59}$ [II] In $62 \%$ of these cases an additional pathogen was also found.

An outpatient study in Arizona found evidence of coccidioidomycosis in $29 \%$ (16-44\%) of 55 cases. ${ }^{60}$ [II]

Studies from south and east Asia found high frequencies of $S$ pneumonia, ${ }^{61}{ }^{62}[\mathrm{Ib}]$ C pneumonia ${ }^{61}{ }^{[\mathrm{Ib}]}$ and Gram-negative bacteria $^{6162}\left[{ }^{[\mathrm{bb}]}\right.$ and Haemophilus influenzae ${ }^{63}[\mathrm{[b]}$ in Thailand. In China, $H$ influenzae was the predominant pathogen in one study, ${ }^{64[\mathrm{Ib}]}$ but $S$ pneumoniae and $M$ pneumoniae in another. ${ }^{65}{ }^{[\mathrm{Ib}]} \mathrm{S}$ pneumoniae followed by $H$ influenzae predominated in Japan, ${ }^{66}$ [II] and $S$ pneumoniae followed by $M$ pneumoniae in Taiwan. ${ }^{67}[\mathrm{Ib}]$

$S$ pneumoniae and Klebsiella pneumoniae were found to be the most frequent causes of CAP in the ICU on an Indian Ocean island. ${ }^{68}[\mathrm{Ib}]$

Table 3 Pathogens which are more common as a cause of community acquired pneumonia in certain geographical regions

\begin{tabular}{|c|c|c|}
\hline Pathogen & Geographical area & References \\
\hline Legionella spp & $\begin{array}{l}\text { Countries bordering the } \\
\text { Mediterranean Sea }\end{array}$ & $27^{[I I]}, 378^{[I I]}$ \\
\hline Coxiella burnetii & North-west Spain & $389^{[11]}$ \\
\hline Coxiella burnetii & Canada & $390^{[11]}$ \\
\hline Klebsiella pneumoniae & South Africa & $391^{[11]}, 392^{[11]}$ \\
\hline Burkholderia pseudomallei & $\begin{array}{l}\text { South-east Asia and } \\
\text { northern Australia }\end{array}$ & $93^{[I I]}, 393^{[I I]}, 394^{[I I]}, 395^{[I I]}$ \\
\hline $\begin{array}{l}\text { Gram-negative enteric } \\
\text { bacilli }\end{array}$ & Italy & $379^{[11]}$ \\
\hline $\begin{array}{l}\text { Mycobacterium } \\
\text { tuberculosis }\end{array}$ & Non-industrialised countries & $93^{[I I]}, 94^{[I I]}$ \\
\hline
\end{tabular}

\subsection{Is the aetiology different in specific population groups?} Elderly subjects

Three UK studies (two using a definition of "elderly" of $>65$ years of age but excluding those aged $>79$ years (quoted in Venkatesan et $\left.a^{69}{ }^{[\mathrm{Ib}]}\right)$ and one study using a definition of $>75$ years $^{37}[\mathrm{ib}]$ ) have reported data on the comparative frequency of pathogens in elderly subjects compared with that in a younger population. The results are combined in fig 3 . For most pathogens their frequency is the same in young as in old subjects, but $M$ pneumoniae and legionella infection are less frequent in elderly people. [Ib] M pneumoniae and other atypical pathogens were found to occur more frequently in patients aged $<60$ years in one other study. ${ }^{70}{ }^{[\mathrm{Ib}]} \mathrm{H}$ influenzae may also be more commonly identified in elderly patients [II]. Gram-negative enteric bacilli were no more common in elderly patients [III], although this has been reported in at least one other study. ${ }^{71}$ [I] No difference in the frequency of pathogens according to age was found in one study of patients with severe CAP. ${ }^{72}$ [III]

One study from Spain compared the aetiology in those aged $>79$ years and $<80$ years and confirmed the previous findings of less $M$ pneumoniae and legionella infection and more aspiration and unknown aetiology in the elderly patients, but did not confirm a greater frequency of $S$ pneumoniae in elderly subjects (fig 4). ${ }^{73}[\mathrm{lb}]$

Patients with chronic obstructive pulmonary disease (COPD)

There are no relevant UK studies and no new data. H influenzae and $M$ catarrhalis may be more frequent. One Danish study directly compared those with and without COPD and found no difference in pathogen frequency; however, numbers were small so real differences may have been missed. ${ }^{74}[\mathrm{II}]$ A Spanish study which focused on patients with COPD but with no control group found a pathogen distribution similar to that described in studies of CAP in the general population. ${ }^{75}[\mathrm{II}]$ A further Spanish study found $S$ pneumoniae, Enterobacteriaceae, Pseudomonas aeruginosa and mixed infections to occur more frequently in those with chronic lung disease. ${ }^{75[\mathrm{Ib}]}$ In one study COPD was found more frequently in patients with bacteraemic pneumococcal pneumonia than other CAPs. ${ }^{76}[\mathrm{Ib}]$ 
Figure 3 Difference in causative pathogens between young and elderly patients. Vertical axis shows the difference in frequency between the young and the elderly groups for pooled data from three UK studies (percentages $\pm 95 \%$ confidence intervals).

$\mathrm{Sp}$, Streptococcus pneumoniae;

$\mathrm{Hi}$, Haemophilus influenzae; Lp, Legionella spp; Sa, Staphylococcus aureus;

Mcat, Moraxella catarrhalis;

GNEB, Gram-negative enteric bacilli;

Mp, Mycoplasma pneumoniae;

$\mathrm{Cp}$, Chlamydophila pneumoniae;

Cpsi, Chlamydophila psittaci; Cb, Coxiella

burnetii; allV, viruses; Flu, influenza

viruses; oth, other organisms; none, no

pathogen identified. Taken from

Venkatesan et $a^{/ 69}$ and Lim et al. ${ }^{37}$
MORE COMMON IN THE ELDERLY

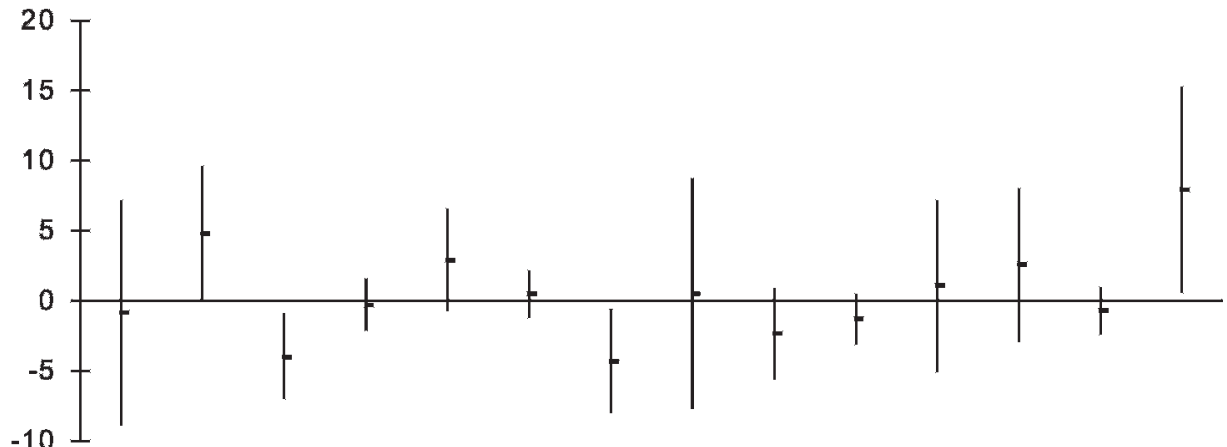

LESS COMMON IN THE ELDERLY

\section{Sp Hi Lp Sa Mcat GNEB Mp Cp Cpsi Cb allV Flu Oth None}

Patients with diabetes

Diabetes was found to be more frequent in patients with bacteraemic pneumococcal pneumonia than in those with either non-bacteraemic pneumococcal pneumonia or all CAPs in one study. ${ }^{76}$ ib] No new data were found.

Nursing home residents

Aspiration, ${ }^{7677}[\mathrm{III}]\left[\mathrm{III}\right.$ Gram-negative enteric bacillii ${ }^{[\mathrm{III}]}$ and anaerobes [IVb] may be more frequent than in matched elderly patients. North American studies, which suggest these differences, may not be relevant to the UK population and healthcare system. Legionella infections and atypical pathogens are uncommon. $^{57}$ [II] [III] The first UK prospective cohort study comparing 40 patients with nursing home acquired pneumonia with 236 adults aged $\geqslant 65$ years with $\mathrm{CAP}^{80}{ }^{[\mathrm{Ib}]}$ found no evidence that the distribution of causative pathogens is different from that in other older adults with CAP. A comparative study from Spain of patients with health care associated pneumonia
(HCAP) which included 25.4\% from a nursing home found a higher frequency of aspiration pneumonia, $H$ influenzae, Gramnegative bacilli and $S$ aureus and a lower frequency of legionella and "no pathogen" in the HCAP group compared with the nonHCAP group. ${ }^{81}[\mathrm{bb]}$

\section{Alcoholic patients}

There are no UK studies. Aspiration, ${ }^{72}$ [II] pneumococcal infection overall, ${ }^{49}\left[\right.$ [b] bacteraemic pneumococcal infection, ${ }^{49} 76$ [Ib] [II] Gram-negative enteric bacilli, ${ }^{71}{ }^{[I I]}$ legionella, ${ }^{82}[\mathrm{IIII}$ atypical pathogens ${ }^{49}{ }^{[\mathrm{Ib}]} \mathrm{C}$ pneumoniae, ${ }^{49}[\mathrm{[b}]$ anaerobes [IVb] and mixed

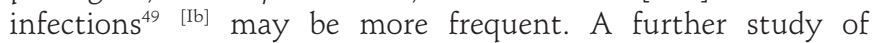
hospitalised patients in Spain found an association with current or ex alcoholism and $S$ pneumoniae infection. ${ }^{83}[\mathrm{bb}]$

Patients on oral steroids

There are no UK studies and no new data. Infection with Legionella species may be more frequent. ${ }^{84}$ [III]
Figure 4 Comparative frequency of identification of pathogens in elderly and young patients in European studies that have contemporaneously applied the same methodology to both groups. Results of four studies totalling 2193 patients (566 elderly patients defined as $>60,>65$ and $>79$ years). ${ }^{69} 7073502$ [II] [II] [II] [II] For each organism, the frequency $( \pm 95 \%$ confidence intervals) in elderly patients is shown in the left bar and in young patients in the right bar.

Sp, Streptococcus pneumoniae;

$\mathrm{Hi}$, Haemophilus influenzae; Lp, Legionella spp; Sa, Staphylococcus aureus; Mcat, Moraxella catarrhalis; GNEB, Gram-negative enteric bacilli; Mp, Mycoplasma pneumoniae; $\mathrm{Cp}$, Chlamydophila pneumoniae; Cpsi, Chlamydophila psittaci; Cb, Coxiella burnetii; allV, viruses; Flu, influenza viruses; oth, other organisms; none, no pathogen identified.

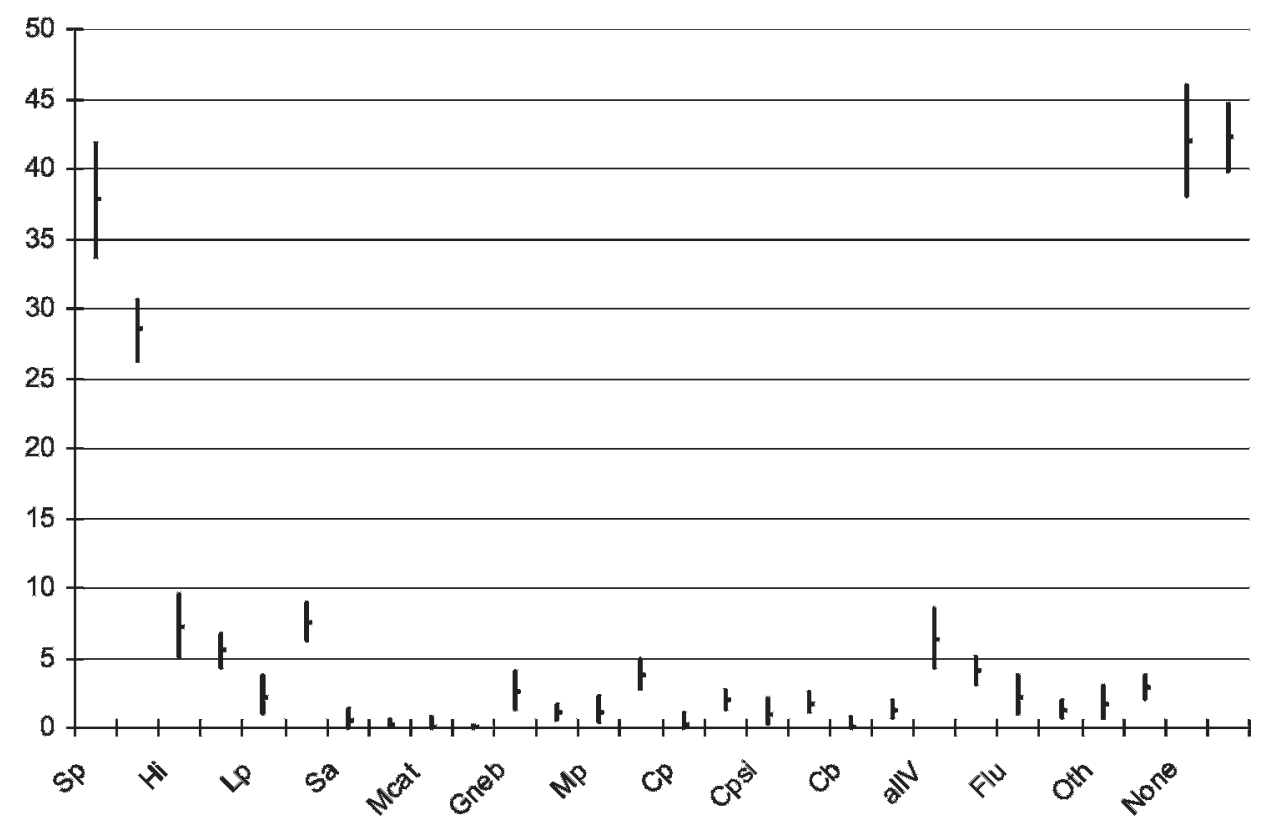




\section{Aspiration pneumonia}

There are no UK studies. Most studies of CAP exclude such patients. Anaerobic bacteria and Gram-negative enteric bacilli may be more common (see the section above on elderly subjects). ${ }^{72} 85$ [III] [III]

\section{Congestive cardiac failure}

A study from Spain suggested a higher frequency of this condition $(31 \%)$ in those with viral pneumonia than in those with mixed (8\%) or pneumococcal pneumonia $(2 \%){ }^{86}[\mathrm{Ib}]$

\subsection{What are the epidemiological patterns of pathogens causing CAP and is this information useful to the clinician?}

\section{Streptococcus pneumoniae}

$S$ pneumoniae occurs most commonly in the winter [II] ${ }^{87}$ [II] Outside the UK, epidemics have occurred in overcrowded settings (eg, mens' shelters and prisons) [II]. ${ }^{87} 88$ [II] [II]

\section{Legionella species}

Legionella infection was most common between June and October, with a peak in August and September in the UK between 1999 and 2005. ${ }^{89}$ [II] Fifty percent of UK cases are related to travel, ${ }^{89} 93 \%$ of these relating to travel abroad. ${ }^{89}$ [II] Clusters of cases are linked to Mediterranean resorts, especially France, Greece, Turkey and Spain, ${ }^{89}[\mathrm{II}]$ but only $23 \%{ }^{90}{ }^{91}[\mathrm{II}]$ of cases occur in clusters. Epidemics occur related to watercontaining systems in buildings. ${ }^{92}$ [II]

\section{Mycoplasma pneumoniae}

Epidemics spanning three winters occur every 4 years in the UK, as shown in fig 5. The apparent decline in reports is probably related to decreased use of complement fixation testing rather than a true decline in frequency.

\section{Chlamydophila pneumoniae}

Epidemics occur in the community and in closed communities. ${ }^{93-95}$ [II] $[\mathrm{II}]$ [II] Its direct pathogenic role as a cause, as opposed to being associated with CAP, is not clear. The lack of a diagnostic gold standard means the frequency is unknown. Serological and $\mathrm{PCR}^{96}{ }^{[\mathrm{Ib}]}$ results are highly variable between assays. Evidence that antibiotic therapy directed against this organism alters the course of the illness is lacking. When identified, other bacterial pathogens (eg, S pneumoniae) are often identified in the same host. ${ }^{97-99}[\mathrm{II}]$ [II] ${ }^{[\mathrm{III}}$ Patients may recover when antibiotics to which $C$ pneumoniae is not sensitive are given. ${ }^{99}[\mathrm{II}]$

\section{Chlamydophila psittaci}

Infection is acquired from birds and animals but human to human spread may occur. [II] Epidemics are reported in relation to infected sources at work (eg, poultry or duck workers). [II] Only $20 \%$ of UK cases have a history of bird contact. ${ }^{100}$ [II]

\section{Coxiella burnetii}

Cases are most common in April to June, possibly related to the lambing and calving season. [II] Epidemics occur in relation to animal sources (usually sheep), but a history of occupational exposure is only present in $7.7 \%$ (95\% CI $6.2 \%$ to $9.4 \%$ ) of cases. $^{101}[\mathrm{III}]$

\section{Staphylococcus aureus}

It is more common in the winter months. Coincident influenzatype symptoms are reported in 39\% (95\% CI $27 \%$ to $53 \%$ ) of cases. $^{63536102[\mathrm{II}]}$ Evidence of coincident influenza virus infection is found in $39 \%$ (95\% CI $17 \%$ to $64 \%$ ) of those admitted to hospital, ${ }^{6536102}$ [II] and 50\% (95\% CI 25\% to 75\%) of those admitted to an ICU. 333442103 [II]

Multiple case reports ${ }^{104-118}[\mathrm{III}]$ and series of 2-11 patients, ${ }^{119-124}$ [II] both from the UK and worldwide, describe episodes of CAP caused by $S$ aureus (either methicillin-sensitive $S$ aureus (MSSA) or MRSA) capable of production of the Panton-Valentine Leucocidin toxin. Severe illness-with high mortality, bilateral lung shadowing and frequent lung cavitation-is common to these reports. No prospective studies have been performed to identify the true frequency of CAP due to this organism, but it appears to be rare at present.

\section{Influenza virus}

Annual epidemics of varying size are seen during the winter months. ${ }^{125}$ [II] Pneumonia complicates $2.9 \%$ (95\% CI $1.4 \%$ to $5.4 \%)$ of cases in the community. ${ }^{126}$ [Ib] The frequency of staphylococcal pneumonia in patients with influenza symptoms is not known. Of adults with CAP admitted to UK hospitals in whom influenza infection is confirmed, 10\% $(95 \%$ CI $4.1 \%$ to $19.5 \%$ ) have coincident $S$ aureus infection. [II] Of those admitted to an ICU, the corresponding figure is $67 \%(95 \%$ CI $35 \%$ to $90 \%) .{ }^{34} 4243103$ [II]

\section{Summary}

- The low frequency of legionella, staphylococcal, $C$ psittaci and $C$ burnetii infection in patients with CAP in both the community and in hospital, together with the likely high frequency of the relevant risk factors (outlined above) in the general population suggests that routine enquiry about such factors is likely to be misleading. [IV]

- Only in those with severe illness where the frequency of legionella and staphylococcal infection is higher may enquiry about foreign travel and influenza symptoms be of predictive value. [IV]

- Knowledge of increased mycoplasma activity in the community during an epidemic period may help guide the clinician to the increased likelihood of mycoplasma infection. [IV]

\section{SECTION 4 CLINICAL FEATURES}

4.1 Can the aetiology of CAP be predicted from clinical features? There have been a large number of publications looking at the possibility of predicting the aetiological agent from the clinical features at presentation; however, while certain symptoms and signs are more common with specific pathogens, none allow accurate differentiation. ${ }^{127} 128$ [II] This led to a suggestion that the term "atypical" pneumonia be abandoned. ${ }^{128}$ As explained in Section 1.3.2, the term "atypical pathogens" remains useful and there is evidence that pleuritic pain is less likely in pneumonia secondary to these agents. ${ }^{129}$

\section{Summary}

- The likely aetiological agent causing CAP cannot be accurately predicted from clinical features. [II]

- The term "atypical" pneumonia should be abandoned as it incorrectly implies that there is a characteristic clinical presentation for patients with infection caused by "atypical" pathogens. [II] 
Figure 5 Laboratory reports to the Infections of infections due to Mycoplasma pneumoniae in England and Wales by date of report, 1990-2008 (4-weekly). Health Protection Agency Centre for

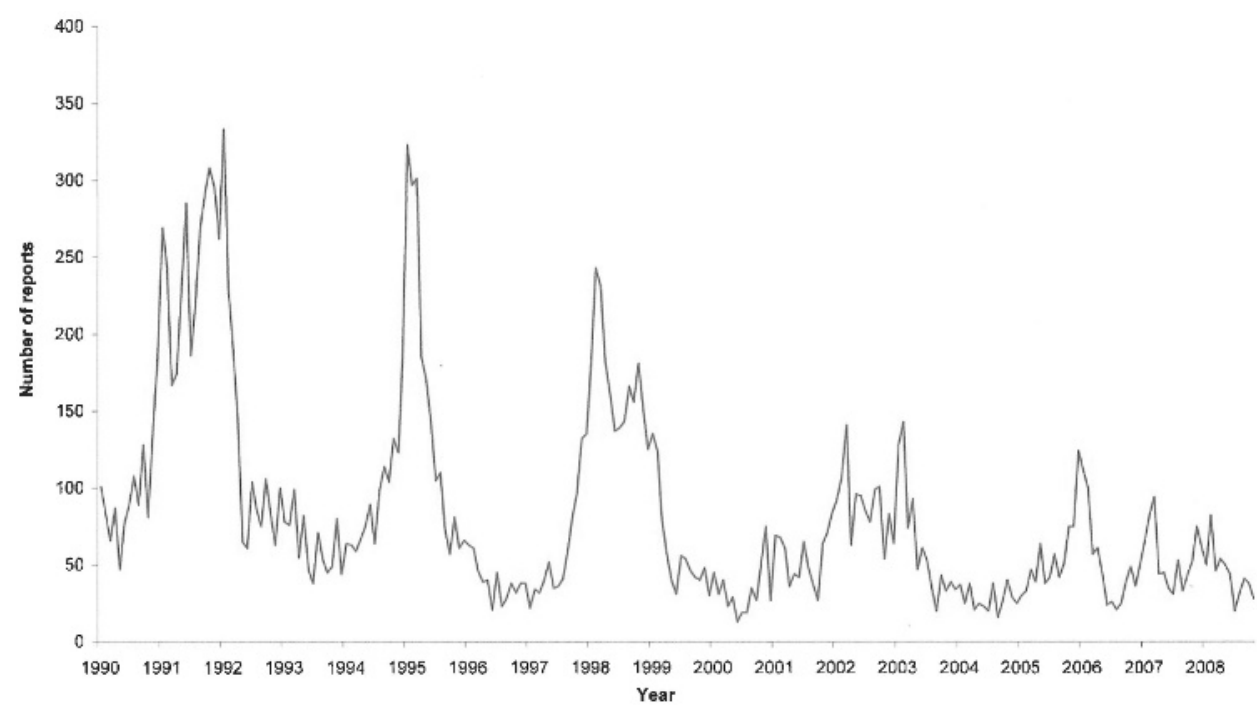

4.2 Specific clinical features of particular respiratory pathogens Clinical features associated with specific pathogens are described below and summarised in box 1 .

\section{Streptococcus pneumoniae}

One study using discriminant function analysis found pneumococcal aetiology to be more likely in the presence of cardiovascular comorbidity, an acute onset, pleuritic chest pain and less likely if patients had a cough or flu-like symptoms or had received an antibiotic before admission. ${ }^{130}$ [III]

Bacteraemic pneumococcal pneumonia was found to be more likely in those patients who had at least one of the following features: female, history of no cough or a non-productive cough, history of excess alcohol, diabetes mellitus or COPD. ${ }^{76}$ [II]

In high severity CAP where patients were admitted to an ICU, clinical features had little value in predicting the aetiological agent with the exception of those patients with fever $\left(>39^{\circ} \mathrm{C}\right)$ or chest pain who were statistically more likely to have pneumococcal pneumonia. ${ }^{44}[\mathrm{II}]$

\section{Legionella pneumophila}

A variety of clinical features have been found to be more common in patients with legionella pneumonia, and yet most

Box 1 Some clinical features reported to be more common with specific pathogens (references are given in the text)

- Streptococcus pneumoniae: increasing age, comorbidity, acute onset, high fever and pleuritic chest pain.

- Bacteraemic S pneumoniae: female sex, excess alcohol, diabetes mellitus, chronic obstructive pulmonary disease, dry cough.

- Legionella pneumophila: younger patients, smokers, absence of comorbidity, diarrhoea, neurological symptoms, more severe infection and evidence of multisystem involvement (eg, abnormal liver function tests, elevated serum creatine kinase).

- Mycoplasma pneumoniae: younger patients, prior antibiotics, less multisystem involvement.

- Chlamydophila pneumoniae: longer duration of symptoms before hospital admission, headache.

- Coxiella burnetii: males, dry cough, high fever. agree that it remains impossible to accurately differentiate on clinical grounds. ${ }^{131} 132$ Studies have reported L pneumophila to be more common in men, ${ }^{133}$ in young patients with lower rates of comorbid illness, ${ }^{134}$ in smokers ${ }^{135}$ and in those who have already received antibiotic therapy. ${ }^{133} 135$ Clinical features which might point towards $L$ pneumophila as an aetiological agent include encephalopathy and other neurological symptoms, gastrointestinal symptoms, more severe infection, elevated liver enzymes, elevated creatine kinase and relatively less frequent upper respiratory tract symptoms, pleuritic chest pain and purulent sputum. $^{82133136}$

\section{Mycoplasma pneumoniae}

One study has compared CAP due to $M$ pneumoniae to patients with pneumococcal or legionella pneumonia. It reported that patients with mycoplasma pneumonia were younger, less likely to have multisystem involvement and more likely to have received an antibiotic before admission. ${ }^{23}$ By contrast, another report found no distinctive clinical features in patients with confirmed $M$ pneumoniae pneumonia. ${ }^{137[\mathrm{II}]}$

\section{Chlamydophila pneumoniae}

A comparative study of patients with $C$ pneumoniae and $S$ pneumoniae pneumonia found the former more likely to present with headaches and a longer duration of symptoms before hospital admission. ${ }^{95}$ [II] A study from Israel reported no distinguishing clinical features for chlamydial pneumonia, except that it affected older patients than pneumococcal and mycoplasma infections. ${ }^{98}[\mathrm{II}]$ A comparison of $C$ pneumoniae and $M$ pneumoniae confirmed the age difference between the groups and stated that, although clinical features could not be used to distinguish between the two, cough, hoarseness and rhinitis were all more common in $M$ pneumoniae pneumonia. ${ }^{138}$ [III] Where $C$ pneumoniae was the only pathogen identified, the illness was generally mild with non-specific symptoms. ${ }^{139}[\mathrm{II}]$

\section{Coxiella burnetii}

CAP due to $C$ burnetii ( $Q$ fever) causes non-specific clinical features. ${ }^{140} 141$ [II] Two reviews of $Q$ fever have reported that infection was more common in younger men and that patients tended to present with dry cough and high fever. ${ }^{142}{ }^{143}$ [III] Epidemiological features are discussed in Section 3. 


\section{Klebsiella pneumoniae}

When compared with $S$ pneumoniae, K pneumoniae was found to affect men more commonly and to present with a lower platelet count and leucopenia. Alcoholics were at particular risk of bacteraemic and fatal Klebsiella pneumonia. ${ }^{144}$ [III]

\section{Some rarer community respiratory pathogens}

CAP caused by Acinetobacter is seen more often in older patients with a history of alcoholism and has a high mortality. ${ }^{145}$ [III]

CAP due to Streptococcus milleri may indicate a dental or abdominal source of infection. ${ }^{146}$ [III] CAP due to viridans streptococci is associated with aspiration. ${ }^{147}[\mathrm{III}]$

\subsection{CAP in elderly patients: are risk factors and clinical features different?}

The classic symptoms and signs of pneumonia are less likely in elderly patients and non-specific features, especially confusion, are more likely. ${ }^{73}{ }^{148-150}$ [II] Comorbid illness occurs more frequently in older patients with CAP and two studies have found the absence of fever to be more common than in younger patients with CAP. ${ }^{128} 151$ [II]

There is a high incidence of aspiration in elderly patients who present with CAP compared with controls (71\% versus $10 \%) .{ }^{152}$ Case-controlled studies of pneumonia acquired in nursing homes have shown that both aspiration and pre-existing comorbid illnesses were more common in nursing homeacquired pneumonia than in others with CAP. ${ }^{148}$ [II] The inpatient mortality rate for nursing home-acquired pneumonia was higher than that for age matched patients with non-nursing home-acquired pneumonia. ${ }^{153}$ [II] The relationship between aetiology of CAP and the age of the patient is discussed in Section 3 .

\section{Summary}

- Elderly patients with CAP more frequently present with non-specific symptoms and have comorbid disease and a higher mortality, and are less likely to have a fever than younger patients. [II]

- Aspiration is a risk factor for CAP in elderly patients, particularly nursing home residents. [II]

\subsection{Aspiration pneumonia}

Aspiration pneumonia embodies the concept of an infectious pneumonic process consequent upon the aspiration of colonised oropharyngeal or gastric contents. However, in practice, such causal linkage is seldom verified. Instead, the term "aspiration pneumonia" is commonly applied to situations when a patient with risk factors for aspiration presents with pneumonia. These risk factors include altered level of consciousness, neurological disorders such as stroke, presence of dysphagia and gastric disorders such as gastro-oesophageal reflux. When a broad definition of aspiration pneumonia is applied to pneumonia study cohorts, up to $10 \%$ of patients admitted to hospital with CAP are identified as having aspiration pneumonia. ${ }^{154}$ [II] This is likely to be an overestimate of the incidence of true aspiration pneumonia.

Studies of the bacteriology of pneumonia in patients with risk factors for aspiration vary widely in relation to inclusion criteria, patient characteristics and microbiological techniques used. $^{44} 72{ }^{154-158}[\mathrm{III}][\mathrm{III}][\mathrm{II}][\mathrm{II}][\mathrm{II}][\mathrm{Ib}][\mathrm{Ib}]$ In true community acquired aspiration pneumonia, multiple pathogens including anaerobes are likely.
SECTION 5 RADIOLOGICAL, GENERAL AND MICROBIOLOGICAL INVESTIGATIONS

\subsection{When should a chest radiograph be performed in the community for patients presenting with suspected CAP?}

In UK practice, most CAP is managed in primary care where access to rapid chest radiography is limited. In this setting, clinicians have to identify the $5-12 \%$ with CAP from the majority with acute non-pneumonic lower respiratory tract infections or other diagnoses. ${ }^{34}[\mathrm{Ib}]$ This challenge is particularly difficult in the presence of comorbid illnesses such as left ventricular failure, chronic lung disease or COPD and in the elderly who frequently present with non-specific symptoms and an absence of chest signs. ${ }^{148}[\mathrm{II}]$

No individual clinical symptom or sign is useful in discriminating CAP from other acute lower respiratory tract infections, ${ }^{159} 160$ [Ia] and there is poor interobserver reliability in eliciting respiratory signs. ${ }^{161}$ [II]

Woodhead et $a^{23}[\mathrm{II}]$ found that $39 \%$ of adults treated with antibiotics for an acute lower respiratory tract infection associated with new focal signs on chest examination had evidence of CAP on chest radiograph compared with $2 \%$ of patients who did not have new focal chest signs. By contrast, Melbye et $a^{162}[\mathrm{II}]$ found that respiratory symptoms and signs were of only minor value in differentiating patients with radiographic pneumonia in a study of 71 patients suspected by their general practitioners of having CAP. The clinical findings reported by the general practitioners to be most suggestive to them of CAP (typical history of cough, fever, dyspnoea and chest pains and lung crackles on examination) had low predictive values; only a short duration of symptoms $(<24 \mathrm{~h})$ was of significant predictive value.

Various prediction rules have been published for the diagnosis of CAP, [II] but generally have shown the need for confirmatory radiographic evidence. Statistical modelling was used by Diehr et $a l^{163[I I]}$ to predict the presence of CAP in 1819 adults presenting to hospital outpatients with acute cough, $2.6 \%$ of whom had CAP on the chest radiograph. The presence of fever $\left(>37.8^{\circ} \mathrm{C}\right)$, raised respiratory rate $(>25$ breaths/min), sputum production throughout the day, myalgia and night sweats, and absence of sore throat and rhinorrhoea were the only clinical features that predicted CAP when included in a diagnostic rule which had 91\% sensitivity and 40\% specificity.

Conversely, a number of studies have suggested that CAP can be safely ruled out in the absence of abnormal vital signs. ${ }^{159} 160$ [Ia] One study compared 350 adults presenting with acute respiratory symptoms to outpatient clinics and the emergency department in California where CAP had been diagnosed on the chest radiograph with an equal number of age-matched controls. The age range of patients was 21-91 years, with an average age of 65 years. The presence of either abnormal vital signs (fever $>38^{\circ} \mathrm{C}$, tachycardia $>100 /$ min and tachypnoea $>20 / \mathrm{min}$ ) or an abnormal physical examination of the chest (crackles, decreased breath sounds, dullness to percussion, wheeze) identified patients with radiographically confirmed CAP with a sensitivity of $95 \%$, negative predictive value of $92 \%$ and specificity of $56 \% .{ }^{164}$ [II] These findings have not been validated in the UK. Despite the age range included in this study, the reduced incidence of classical features of pneumonia and fever with increasing age at presentation (see Section 4.4) should be borne in mind when applying these results to elderly patients.

In practice, general practitioners manage the vast majority of patients pragmatically at first presentation. The important decision in patients presenting with a lower respiratory tract 
infection, or suspected CAP, is deciding whether to use an antibiotic, which one and how ill the patient is. Labelling the illness as pneumonia is less important. ${ }^{165}$

\section{Recommendations}

It is not necessary to perform a chest radiograph in patients with suspected CAP unless:

- The diagnosis is in doubt and a chest radiograph will help in a differential diagnosis and management of the acute illness. [D]

- Progress following treatment for suspected CAP is not satisfactory at review. [D]

- The patient is considered at risk of underlying lung pathology such as lung cancer (see Section 5.6). [D]

\subsection{When should a chest radiograph be performed in hospital for patients presenting with suspected CAP?}

A chest radiograph is the cornerstone to confirming a diagnosis of CAP. In patients ill enough to require hospital referral for suspected CAP, a chest radiograph is essential to establishing the diagnosis of CAP or an alternative diagnosis, and therefore in guiding management decisions.

Antibiotic treatment of patients with suspected CAP prior to, or without, confirmation by chest radiography potentially leads to inappropriate and excessive antibiotic use.

The committee felt that the Department of Health's " 4 hour from presentation to admission, transfer or discharge" target for patients admitted to emergency departments represented a practice standard that should apply to all patients presenting to hospital (via the emergency department or acute medical unit) with suspected CAP. ${ }^{15}$

\section{Recommendation}

- All patients admitted to hospital with suspected CAP should have a chest radiograph performed as soon as possible to confirm or refute the diagnosis. [D] The objective of any service should be for the chest radiograph to be performed in time for antibiotics to be administrated within $4 \mathrm{~h}$ of presentation to hospital should the diagnosis of CAP be confirmed.

\subsection{Are there characteristic features that enable the clinician to predict the likely pathogen from the chest radiograph?}

There are no characteristic features on the chest radiograph in CAP that allow confident prediction of the causative organism. $^{98}{ }^{166-168}$ [III] The lower lobes are affected most commonly, regardless of aetiology.

Multilobe involvement ${ }^{169}$ [II] at presentation and pleural effusions were more likely at presentation in bacteraemic pneumococcal pneumonia than in non-bacteraemic pneumococcal pneumonia or legionella pneumonia. Homogenous shadowing was less common in mycoplasma pneumonia than in the other types. Lymphadenopathy was noted in some cases of mycoplasma infections but not in the other types of infection. CAP due to $S$ aureus appears to be more likely to present with multilobar shadowing, cavitation, pneumatoceles or spontaneous pneumothorax. ${ }^{170}$ [III] $\mathrm{K}$ pneumoniae has been reported to produce chest radiograph changes with a predilection for upper lobes (especially the right). ${ }^{171}{ }^{[\mathrm{II}]} \mathrm{A}$ bulging interlobar fissure and abscess formation with cavitation have also been reported, although the former is probably just a reflection of an intense inflammatory reaction that can occur in any severe infection such as pneumonia due to $S$ aureus. ${ }^{170}[\mathrm{III}]$

\section{Summary}

- There are no characteristic features of the chest radiograph in CAP that allows a confident prediction of the likely pathogen. [II]

\subsection{What is the role of CT lung scans in CAP?}

There are few data on the role of high-resolution CT lung scans in CAP. A small study has reported that high-resolution CT scans may improve the accuracy of diagnosing CAP compared with chest radiography alone. ${ }^{172}[\mathrm{II}]$ Similarly, CT lung scans have improved sensitivity compared with standard chest radiographs in patients with mycoplasma pneumonia. ${ }^{173}$ [II] CT lung scans may be useful in subjects where the diagnosis is in doubt ${ }^{174}[\mathrm{III}]$ but, in general, there is little role for CT scanning in the usual investigation of CAP.

With regard to aetiology, one study has reported a difference in CT appearances in 18 patients with CAP due to bacterial infections compared with 14 patients with atypical pathogens. ${ }^{175}$ [III]

\section{Summary}

- CT scanning currently has no routine role in the investigation of CAP. [II]

\subsection{How quickly do chest radiographs improve after CAP?}

Radiographic changes resolve relatively slowly after CAP and lag behind clinical recovery. In one study, complete resolution of chest radiographic changes occurred at 2 weeks after initial presentation in $51 \%$ of cases, in $64 \%$ by 4 weeks and $73 \%$ at 6 weeks. ${ }^{176}$ Clearance rates were slower in elderly patients, those with more than one lobe involved at presentation, smokers and inpatients rather than outpatients. Multivariate analysis showed that only age and multilobe involvement were independently related to rate of clearance. Age was also a major factor influencing rate of radiographic recovery in the BTS multicentre CAP study. ${ }^{\left[{ }^{[b]}\right]}$ A study of patients over 70 years of age showed $35 \%, 60 \%$ and $84 \%$ radiographic resolution at 3,6 and 12 weeks, respectively. ${ }^{177}{ }^{[I]}$ C-reactive protein (CRP) levels $>200 \mathrm{mg} / \mathrm{l}$ were also linked to slower radiographic resolution. ${ }^{178}[\mathrm{III}]$ When chest radiographs of patients with bacteraemic pneumococcal pneumonia were followed, only $13 \%$ had cleared at 2 weeks and $41 \%$ at 4 weeks. ${ }^{179}{ }^{[I I I]}$ Pneumonias caused by atypical pathogens clear more quickly. The clearance rate has been reported to be faster for mycoplasma pneumonia than for legionella or pneumococcal pneumonia, which may take 12 weeks or more. ${ }^{166}{ }^{[I I I]}$ In a series of patients with $C$ burnetii pneumonia, $81 \%$ of the chest radiographs had returned to normal within 4 weeks. ${ }^{143}$ [III]

Radiographic deterioration after admission to hospital was more common with legionella (65\% of cases) and bacteraemic pneumococcal pneumonia (52\%) than with non-bacteraemic pneumococcal $(26 \%)$ or mycoplasma pneumonia $(25 \%) .{ }^{166}[\mathrm{III}]$ Residual pulmonary shadowing was found in over $25 \%$ of cases of legionella and bacteraemic pneumococcal cases. Deterioration after admission has also been reported in over half of cases of $S$ aureus pneumonia. ${ }^{170}[\mathrm{III}]$ Radiographic deterioration after hospital admission appears to be commoner in older patients (aged $\geqslant 65$ years). ${ }^{151}[\mathrm{II}]$

\section{Summary}

- Radiological resolution often lags behind clinical improvement from CAP, particularly following legionella and bacteraemic pneumococcal infection. [III] 
- Pneumonia caused by atypical pathogens clears more quickly than pneumonia caused by bacterial infection. [III]

- Radiological resolution is slower in elderly patients and where there is multilobar involvement. [Ib]

\subsection{When should the chest radiograph be repeated during recovery and what action should be taken if the radiograph has not returned to normal?}

Repeat chest radiographs are probably often ordered unnecessarily following CAP. ${ }^{180}$ [IVa] Although it has become usual practice to repeat the chest radiograph on hospital discharge and again at "routine" hospital clinic follow-up at around 6 weeks later, there is no evidence on which to base a recommendation regarding the value of this practice in patients who have otherwise recovered satisfactorily.

The main concern is whether the CAP was a complication of an underlying condition such as lung cancer. This concern will depend on a variety of factors such as age, smoking status, preexisting conditions such as COPD and the clinical condition of the patient. In a study of 236 adults presenting to their general practitioner with a clinical diagnosis of CAP, 10 were found to have underlying lung cancer on investigation. There was a high frequency of lung cancer in older smokers (6 of $36(17 \%)$ smokers aged $>60$ years), suggesting that a chest radiograph was particularly indicated in this group of patients with CAP in the community. ${ }^{23}$ [II] Studies of CAP in hospital often exclude patients found to have lung cancer, making it difficult to assess how frequently lung cancer presents acutely with CAP. In one study of 162 adults hospitalised with suspected CAP, the diagnosis was accepted in only 127, 10 (6\%) of the 162 being found to have cancer. ${ }^{36}{ }^{[I I]}$ Another study found only 13 (1.3\%) of 1011 patients hospitalised with CAP to have an underlying lung cancer on investigation. ${ }^{181}$ [III] Eight of these were detected on the admission chest radiograph and the others were detected because of unsatisfactory clinical recovery. They concluded that a convalescent radiograph was useful in detecting occult lung cancer only if signs or symptoms persisted after a month or so.

The practice of performing bronchoscopy in patients admitted to hospital with CAP prior to hospital discharge has been investigated. ${ }^{182}\left[{ }^{[I I]}\right.$ In patients aged $>50$ years or who were current or ex-smokers, $14 \%$ were found to have an abnormality at bronchoscopy (11\% had a bronchial carcinoma diagnosed).

\section{Recommendations}

- The chest radiograph need not be repeated prior to hospital discharge in those who have made a satisfactory clinical recovery from CAP. [D]

- A chest radiograph should be arranged after about 6 weeks for all those patients who have persistence of symptoms or physical signs or who are at higher risk of underlying malignancy (especially smokers and those aged $>50$ years) whether or not they have been admitted to hospital. [D]

- Further investigations which may include bronchoscopy should be considered in patients with persisting signs, symptoms and radiological abnormalities at around 6 weeks after completing treatment. [D]

- It is the responsibility of the hospital team to arrange the follow-up plan with the patient and the general practitioner for those patients admitted to hospital (see Section 7.5). [D]
5.7 What general investigations should be done in a patient with suspected CAP in the community?

General investigations are performed to assess severity (see Section 6), to assess the impact on or to detect the presence of any comorbid disease, to provide some pointer to the particular aetiological agent or group of pathogens, identify complications and to monitor progress (see Section 9).

It may be appropriate to perform investigations in selected patients, especially if there is delayed improvement on review. However, no firm recommendations can be offered. It is a matter of clinical judgement.

\section{Recommendations}

- General investigations are not necessary for the majority of patients with CAP who are managed in the community. [C] Pulse oximeters allow for simple assessment of oxygenation. General practitioners, particularly those working in out-of-hours and emergency assessment centres, should consider their use (see Section 7.1). [D]

- Pulse oximetry should be available in all locations where emergency oxygen is used. [D]

\subsection{What general investigations should be done in patients admitted to hospital?}

Apart from the chest radiograph essential for diagnosis, the only other simple non-microbiological tests that influence immediate management are the urea, which informs severity assessment, and oxygen saturation, which affects supportive management and track and trigger systems in accordance with the BTS guideline for emergency oxygen use in adult patients. ${ }^{183}$

In addition, it is normal practice to take blood for a full blood count, urea and electrolytes, liver function tests and CRP. These often help to identify important underlying or associated pathologies including renal or hepatic disease and haematological or metabolic abnormalities.

A white cell count of $>15 \times 10^{9} / 1$ strongly implicates a bacterial (particularly pneumococcal) aetiology, although lower counts do not exclude a bacterial cause. ${ }^{184}$ [III] A white cell count of $>20 \times 10^{9} / 1$ or $<4 \times 10^{9} / 1$ is an indicator of severity (see Section $6)$.

Considering the role of CRP in the diagnosis of CAP, a prospective study performed in Spain reported a $96 \%$ specificity for CAP using a threshold CRP level of $>100 \mathrm{mg} / \mathrm{l}^{185}$ [II] Criticisms of this study are the small number of patients in one group and the fact that patients with infective exacerbations of COPD were excluded. Another study showed that a raised CRP level on admission is a relatively more sensitive marker of pneumonia than an elevated temperature or raised white cell count. All patients with CAP had CRP levels $>50 \mathrm{mg} / \mathrm{l}$ and $75 \%$ of patients had levels $>100 \mathrm{mg} / 1 .^{186}[\mathrm{II}]$ In the same paper it was reported that a CRP level of $>100 \mathrm{mg} / \mathrm{l}$ helped to distinguish CAP from acute exacerbations of COPD. Another group found that only $5 \%$ of patients admitted with CAP had CRP levels $<50 \mathrm{mg} / 1{ }^{187}$ [III] Although not yet widely available, a bedside finger-prick CRP test has been used to predict CAP in 168 patients presenting with acute cough and, at a cut-off of $40 \mathrm{mg} / \mathrm{l}$, was found to have a sensitivity of $70 \%$ and a specificity of $90 \%$ independent of any clinical characteristics. ${ }^{188}$ [II] CRP levels are generally higher in patients who have not received antibiotic therapy before admission. ${ }^{186}[\mathrm{II}]$

With regard to predicting the microbial aetiology of CAP, higher CRP levels have been associated with pneumococcal 
pneumonia (especially if complicated by bacteraemia) compared with mycoplasma or viral pneumonias, ${ }^{189}[\mathrm{III}]$ and in legionella pneumonia compared with all other identified single aetiologies. ${ }^{190}[\mathrm{II}]$

Data relating to CRP as a marker of severity and of treatment failure are discussed in Sections 6.2.1 and 7.3, respectively).

There are a number of studies examining the role of other biomarkers in CAP such as procalcitonin, however these assays are not currently widely available.

\section{Summary}

- The published evidence to date suggests that measurement of CRP on admission may be helpful in distinguishing pneumonia from other acute respiratory illnesses. [III]

\section{Recommendations}

All patients should have the following tests performed on admission:

- Oxygenation saturations and, where necessary, arterial blood gases in accordance with the BTS guideline for emergency oxygen use in adult patients. [B+]

- Chest radiograph to allow accurate diagnosis. [B+]

- Urea and electrolytes to inform severity assessment. $[\mathrm{B}+]$

- CRP to aid diagnosis and as a baseline measure. [B+]

- Full blood count. [B-]

- Liver function tests. [D]

\subsection{Why are microbiological investigations performed in patients with CAP?}

Establishing the microbial cause of CAP is useful for several reasons:

- Identification of pathogens and antibiotic sensitivity patterns permits selection of optimal antibiotic regimens. To date there has been a habit to continue broad-spectrum empirical antibiotics even if a specific pathogen has been identified. However, with the increasing problem of antibiotic resistance and HCAIs such as $C$ difficile infection, the balance has now swung towards focusing down antibiotic therapy whenever possible.

- Targeted and narrow-spectrum antibiotic therapy limits drug costs, the threat of antibiotic resistance and adverse drug reactions such as $C$ difficile-associated diarrhoea.

- Specific pathogens have public health or infection control significance, including legionella, psittacosis, C burnetii, influenza $\mathrm{A}$ and multiresistant organisms. Patients with these infections should be identified quickly so that appropriate treatment and control measures can be implemented.

- Microbiological investigations allow monitoring of the spectrum of pathogens causing CAP over time. This allows trends regarding aetiology and antibiotic sensitivity to be tracked for public health needs.

Unfortunately, microbiological investigations are insensitive and often do not contribute to initial patient management. ${ }^{191}$ [III] In detailed prospective aetiology studies the microbial cause is not found in $25-60 \%$ of patients, ${ }^{23} 192[\mathrm{III}[\mathrm{III}]$ and the yield is even lower in routine hospital practice. ${ }^{193} 194$ [III] [III] More recent studies including the use of PCR and antigen detection techniques have not generally increased the proportion of patients with a specific aetiological diagnosis. ${ }^{195} 196$ [II] $[\mathrm{II}]$ In contrast, one recent prospective study of 105 adults with CAP ${ }^{197}$ ${ }^{[I I]}$ comparing a multiplex real-time PCR for a range of "atypical pathogens" and respiratory viruses with conventional methods reported a microbiological diagnosis in 80 patients $(76 \%)$ using the real-time PCR compared with 52 patients (49.5\%) using conventional methods. However, no urine antigen testing for either legionella or pneumococcal infection was included in the study, and most of the increase in diagnostic yield obtained was due to enhanced detection of rhinoviruses and coronaviruses. Nevertheless, such studies point the way forward for improving aetiological diagnosis in CAP.

Several studies ${ }^{198-201}$ [II] [II] [II] [II] have examined the positivity rate of routine microbiological investigations (blood and sputum cultures) for patients with CAP. These studies provide further evidence that the overall sensitivity of such tests in CAP is low, particularly for patients with low severity CAP and no comorbid disease, and for those who have received antibiotic therapy prior to admission. One study ${ }^{200}$ demonstrated a direct correlation between the severity of pneumonia (using the Fine Pneumonia Severity Index (PSI)) and blood culture positivity rate, and questioned the value of routine blood cultures for patients in PSI risk classes I-III (ie, low severity). However, another study found poor correlation of blood culture positivity with the PSI among patients hospitalised with CAP. ${ }^{201}[\mathrm{II}]$

\section{Recommendations}

- Microbiological tests should be performed on all patients with moderate and high severity CAP, the extent of investigation in these patients being guided by severity. [D]

- For patients with low severity CAP the extent of microbiological investigations should be guided by clinical factors (age, comorbid illness, severity indicators), epidemiological factors and prior antibiotic therapy. $[\mathrm{A}-]$

- Where there is clear microbiological evidence of a specific pathogen, empirical antibiotics should be changed to the appropriate pathogen-focused agent unless there are legitimate concerns about dual pathogen infection. [D]

\subsection{What microbiological investigations should be performed in patients with suspected CAP in the community?}

Comments about the pros and cons of different microbiological investigations are given below in Section 5.11. Many of these investigations will not be appropriate for patients with CAP managed in the community. Such patients are not usually severely ill, are at low risk of death and delays in transport of specimens to the laboratory reduces the yield of bacterial pathogens (especially $S$ pneumoniae) from sputum cultures. Results are often received too late by the general practitioner to be of much practical value in initial management.

\section{Recommendations}

- For patients managed in the community, microbiological investigations are not recommended routinely. [D]

- Examination of sputum should be considered for patients who do not respond to empirical antibiotic therapy. [D]

- Examination of sputum for Mycobacterium tuberculosis should be considered for patients with a persistent productive cough, especially if malaise, weight loss or night sweats, or risk factors for tuberculosis (eg, ethnic origin, social deprivation, elderly) are present. [D] 
Table 4 Recommendations for the microbiological investigation of community acquired pneumonia (CAP)

Pneumonia severity (based on clinical judgement supported by severity scoring tool)

Low severity

(eg, CURB65 $=0-1$ or CRB-65 score $=0,<3 \%$ mortality)

Low severity

(eg, CURB65 $=0-1,<3 \%$ mortality) but admission indicated for reasons other than pneumonia severity (eg, social reasons)

Moderate severity (eg, CURB65 = 2, 9\% mortality)

High severity

(eg, CURB65 = 3-5, 15-40\% mortality)

Treatment site

Preferred microbiological tests

Home

Hospital

Hospital

(a)

(n) 
- If a diagnosis of CAP has been definitely confirmed and a patient has low severity pneumonia with no comorbid disease, then blood cultures may be omitted. $[\mathrm{A}-]$

\subsubsection{Sputum cultures}

Sputum cultures may identify the causative agent in CAP including unexpected or antibiotic-resistant pathogens such as $S$ aureus or antimicrobial-resistant pneumococci. Routine sputum cultures are, however, neither very sensitive nor specific ${ }^{207}$ [Ia] and often do not contribute to initial patient management. ${ }^{208}[\mathrm{II}]$

Problems include:

- The inability of patients to produce good specimens.

- Prior exposure to antibiotics.

- Delays in transport and processing.

- Difficulty in interpretation due to contamination of the sample by upper respiratory tract flora, which may include potential pathogens such as S pneumoniae and "coliforms" (especially in patients already given antibiotics).

\section{Recommendations}

- Sputum samples should be sent for culture and sensitivity tests from patients with CAP of moderate severity who are able to expectorate purulent samples and have not received prior antibiotic therapy. Specimens should be transported rapidly to the laboratory. [A-]

- Culture of sputum or other lower respiratory tract samples should also be performed for all patients with high severity CAP or those who fail to improve. [A-]

- Sputum cultures for Legionella spp should always be attempted for patients who are legionella urine antigen positive in order to provide isolates for epidemiological typing and comparison with isolates from putative environmental sources. [D]

\subsubsection{Sputum Gram stain}

The value of performing a Gram stain on expectorated sputum has been widely debated. A meta-analysis review concluded that the sensitivity and specificity of sputum Gram stain in patients with CAP varied substantially in different settings. ${ }^{209}$ [Ia] The presence of large numbers of Gram-positive diplococci in purulent samples from patients with CAP can indicate pneumococcal pneumonia. ${ }^{210}{ }^{[I]}$ A study of 1669 consecutive adult patients with CAP found that good quality sputum samples with a predominant bacterial morphotype on Gram stain (ie, the test was useful) were obtained from only $14.4 \%$ of patients overall and, while Gram-positive diplococci as the predominant morphotype was highly specific for $S$ pneumoniae, no severity subgroup of patients (assessed using the PSI) could be identified in whom the test would be of greater utility. ${ }^{211}$ [III] A similar study ${ }^{212}$ [III] of 347 patients with CAP concluded that Gram stain of sputum was useful in guiding microbiological diagnosis in just $23 \%$ of patients and unreliable in patients who had received antimicrobial treatment prior to sample collection. There are many factors which need to be borne in mind when considering the reliability and usefulness of Gram stain results. These are summarised below:

\section{Advantages}

- Quick and relatively inexpensive.

- Can assess quality of samples (cytological content) with rejection of poor quality samples.
- Can aid the interpretation of culture results and occasionally give an early indication of possible aetiology.

\section{Disadvantages}

- Strict criteria for interpretation require appropriate operator training.

- Validity of results is directly related to the experience of the interpreter. ${ }^{213}$ [II]

- Sputum Gram stain correlates poorly with culture results in conditions other than CAP. ${ }^{214}{ }^{[\mathrm{II}]}$ This poses practical difficulties for laboratories that frequently have to interpret results with little or no clinical information.

- Lack of availability: a recent survey of diagnostic microbiology laboratories in England and Wales ${ }^{215}$ [III] revealed that, of 138 respondents, 53 laboratories (38\%) do not provide a sputum Gram stain service at all and, of the remainder, 52 laboratories (38\%) do so only on special request. Thus, ready availability of sputum Gram stain cannot be assumed. This lack of availability reflects the opinion of many microbiologists that sputum examination is rarely helpful in the diagnosis of CAP.

\section{Recommendations}

- Clinicians should establish with local laboratories the availability or otherwise of sputum Gram stain. Where this is available, laboratories should offer a reliable Gram stain for patients with high severity CAP or complications as occasionally this can give an immediate indicator of the likely pathogen. Routine performance or reporting of sputum Gram stain on all patients is unnecessary but can aid the laboratory interpretations of culture results. [B-]

- Samples from patients already in receipt of antimicrobials are rarely helpful in establishing a diagnosis. [B-]

- Laboratories performing sputum Gram stains should adhere to strict and locally agreed criteria for interpretation and reporting of results. $[\mathrm{B}+]$

\subsubsection{Other tests for Streptococcus pneumoniae}

Pneumococcal antigen detection

Pneumococcal antigens can be detected in various body fluids during active pneumococcal infection, including sputum, pleural fluid, serum and urine. Antigen detection is less affected by prior antibiotic therapy and the detection of antigenaemia has a correlation with clinical severity. ${ }^{216}[\mathrm{IVb}]$

A commercial immunochromatographic strip test (BINAX NOW) for detection of pneumococcal antigen in urine has been introduced in the last few years and been widely taken up. Numerous studies ${ }^{217-222}$ [II] [II] [II] [II] [II] [III] have evaluated positively the clinical and diagnostic utility and generally good sensitivity and specificity of the pneumococcal urine antigen test in the diagnosis of pneumococcal pneumonia in adults. The studies have shown the usefulness of this assay in determining the aetiology of CAP, with significantly greater sensitivity rates than routine blood or sputum cultures. In addition, the test remains positive in $80-90 \%$ of patients for up to 7 days after starting antimicrobial treatment, ${ }^{223}[\mathrm{II}]$ and may also be applied to other relevant sample types such as pleural fluid. ${ }^{224}$ [III]

\section{Pneumococcal PCR}

Many polymerase chain reaction (PCR)-based methods for detection of pneumococcal DNA in clinical samples have been published, varying in precise methodology and the specific 
pneumococcal DNA target(s) sought. However, relatively few studies report comprehensive clinical-as opposed to analytical sensitivity-evaluations of pneumococcal PCRs in the diagnosis of CAP. One retrospective study ${ }^{225}$ [III] compared three different PCR methodologies for use on EDTA blood samples from 175 bacteraemic patients collected at hospital admission (95 pneumococcal bacteraemia and 80 with bacteraemia due to other organisms). The best sensitivity obtained was $45 \%$ versus the gold standard of a positive blood culture for $S$ pneumoniae. The specificity of all three methods was good at $97-100 \%$. The authors concluded that blood PCR offers no advantage over conventional blood culture for pneumococcal diagnosis in bacteraemic patients and is unlikely to be sufficiently sensitive for diagnosis of non-bacteraemic pneumococcal pneumonia. Pneumococcal PCR has also been applied to sputum and other respiratory tract samples. However, obtaining a good quality sputum sample, as described above, remains problematic and, more importantly, PCR is not readily able to distinguish colonisation from infection of the respiratory tract. ${ }^{226}$ [III] Pneumococcal PCR has little to offer for the diagnosis of CAP at this time, being insufficiently sensitive and specific for routine use.

\section{Recommendations}

- Pneumococcal urine antigen tests should be performed for all patients with moderate or high severity CAP. [A-]

- A rapid testing and reporting service for pneumococcal urine antigen should be available to all hospitals admitting patients with CAP. $[\mathrm{B}+]$

\subsubsection{Tests for Legionnaires' disease}

Legionella pneumonia can be severe and carries a significant mortality. Prompt diagnosis is important both for patient management and for public health investigations. Risk factors for legionella infection include recent travel (within 10 days of onset), certain occupations, recent repair to domestic plumbing systems and immunosuppression.

\section{Urine antigen detection}

Detection of $L$ pneumophila urinary antigen by enzyme immunoassay (EIA) is established as a highly specific (>95\%) and sensitive $(\sim 80 \%)$ test $^{227}[\mathrm{III}]$ for the detection of infections caused by $L$ pneumophila serogroup 1 , the commonest cause of sporadic and travel CAP cases in the UK. Rapid results can be obtained at an early stage of the illness, and this is a valuable method in the early diagnosis of legionella infection. ${ }^{92}$ [III] It is now widely applied in high severity CAP. A recent survey of diagnostic microbiology laboratories in England and Wales ${ }^{215}$ [III] revealed that, of 138 respondents, 136 laboratories (99\%) offered this test for patients with CAP.

One study looked at the value of rapid legionella urine antigen testing in a large outbreak of Legionnaires' disease caused by $L$ pneumophila serogroup 1 in Holland. ${ }^{228}$ [III] This showed a higher test positivity rate for patients with severe legionella infection. The authors also demonstrated that the results of rapid testing could be used to start early legionella appropriate antibiotic management resulting in an improved outcome, as shown by reducing both mortality and the need for intensive care. In another prospective study of sporadic CAP in adults, the early detection of urine legionella antigen positively influenced the management of seven of nine patients in whom it was detected. ${ }^{37}[\mathrm{Ib}]$
There are several commercial assays available, including a rapid immunochromatographic test. These assays principally detect infection with $L$ pneumophila serogroup 1 . They do not reliably detect antigen from other serogroups or legionella species which can cause infection in immunocompromised patients who may present with CAP, or recently hospitalised patients. This has particular significance in nosocomial infection. In one study of culture confirmed cases, while the sensitivity of commercially available urine antigen tests was $93.7 \%$ for travel-associated cases and $86.5 \%$ for communityacquired cases, it was only $45 \%$ for nosocomial cases. ${ }^{229}$ [III]

\section{Legionella direct immunofluorescence tests}

$L$ pneumophila can be detected by direct immunofluorescence (DIF) on invasive respiratory samples such as bronchial aspirates. L pneumophila specific reagents should be used, and not hyperimmune rabbit antisera which are poorly specific. The value of performing DIF on expectorated sputum samples is less well established. A considerable degree of laboratory expertise is required for processing and interpretation and, in view of the widespread availability of urine antigen tests for legionella diagnosis, the use of DIF has declined in recent years in the UK.

\section{Culture}

The culture of legionella from clinical samples (principally respiratory samples, including sputum) is very important and every effort should be made to diagnose by this method. Culture is $100 \%$ specific and is the only reliable method of detecting infection with non-pneumophila legionella species. Culture is also valuable for epidemiological investigations, allowing phenotypic and genotypic comparison of clinical and environmental legionella strains.

Problems with culture include: the inability of many patients with legionella pneumonia to produce sputum samples; prior antibiotic therapy; laboratory time and cost in processing samples; and lack of rapid results (legionella cultures need to be incubated for up to 10 days). Few laboratories will set up legionella cultures on respiratory samples unless specifically requested to do so. Culture should always be attempted from urine antigen positive patients and in suspected nosocomial legionella infection.

\section{Serology}

The diagnosis by determination of antibody levels was the mainstay of diagnosis of legionella pneumonia in the past. Serological assays previously employed in the UK were highly specific, although false positive results due to a serological crossreaction may occur in patients with recent Campylobacter infection. ${ }^{230}$ [II] Serological reagents for legionella diagnosis are no longer available from the Health Protection Agency. Their place has been filled by a number of commercially available serological assays of varying sensitivity and specificity. It is currently recommended that, where a diagnosis of legionella infection relies solely on the results of serological testing, the sample should be referred to a reference laboratory for confirmation.

\section{PCR}

Detection of legionella DNA by PCR from respiratory samples is still only available as a reference laboratory or research tool, although it is becoming more widely available. 


\section{Recommendations}

- Investigations for legionella pneumonia are recommended for all patients with high severity CAP, for other patients with specific risk factors and for all patients with CAP during outbreaks. [D]

- Legionella urine antigen tests should be performed for all patients with high severity $\mathrm{CAP}$. $[\mathrm{B}+]$

- A rapid testing and reporting service for legionella urine antigen should be available to all hospitals admitting patients with CAP. $[\mathrm{B}+]$

- As the culture of legionella is very important for clinical reasons and source identification, specimens of respiratory secretions, including sputum, should be sent from patients with high severity CAP or where Legionnaires' disease is suspected on epidemiological or clinical grounds. [D] The clinician should specifically request legionella culture on laboratory request forms.

- Legionella cultures should be routinely performed on invasive respiratory samples (eg, obtained by bronchoscopy) from patients with CAP. [D]

- For all patients who are legionella urine antigen positive, clinicians should send respiratory specimens such as sputum and request legionella culture. [D] This is to aid outbreak and source investigation with the aim of preventing further cases.

\subsubsection{Tests for Mycoplasma pneumoniae}

The mainstay of conventional diagnosis at the present time is by serology, although diagnosis by specific PCR is likely to become increasingly available. Culture of $M$ pneumoniae is generally not available in diagnostic laboratories.

The commonest serological assay used historically was the complement fixation test (CFT), but there are various alternative assays such as microparticle agglutination and EIAs. The CFT is still regarded as the "gold standard" to which other assays have been compared, although it does lack sensitivity and specificity. A comparison of various mycoplasma antibody assays (including IgM and CFT tests) concluded that there is no single assay with significantly better sensitivity and specificity than the others. ${ }^{231}[$ [II] Elevated CFT titres are usually detected no earlier than 10-14 days after the onset of mycoplasma infection, but the insidious onset and slow progression of symptoms means that many patients admitted to hospital with mycoplasma CAP have elevated titres on or shortly after admission.

One study ${ }^{232}$ [II] compared 12 commercially available serodiagnostic assays for $M$ pneumoniae with the CFT using serum samples from patients with PCR-confirmed $M$ pneumoniae infection and known onset dates. There were wide variations between the tests in sensitivity and specificity, CFT being the most specific (97\%) although not especially sensitive (65\%). The authors concluded that there are currently few commercial serological assays for the detection of $M$ pneumoniae infections with appropriate performances in terms of sensitivity and specificity, and that PCR is likely to become increasingly important in specific diagnosis. In one series of patients with CAP, ${ }^{197}$ [II] application of PCR to respiratory tract samples doubled the detection rate of $M$ pneumoniae infection versus serological testing alone from 5 to 10 of the 105 patients studied. Another study ${ }^{233}$ [III] described the application of realtime PCR to acute phase serum samples from patients with serologically (CFT) diagnosed mycoplasma pneumonia. Serum samples from 15 of 29 patients (52\%) were $M$ pneumoniae PCR positive, suggesting that serum PCR as opposed to respiratory tract sample PCR - with inherent issues of specimen quality-is worthy of further consideration. PCR diagnosis is already available in some centres in the UK, will become increasingly available, and is likely to replace serodiagnosis in the longer term.

\section{Recommendations}

- Where available, PCR of respiratory tract samples such as sputum should be the method of choice for the diagnosis of mycoplasma pneumonia. [D]

- In the absence of a sputum or lower respiratory tract sample, and where mycoplasma pneumonia is suspected on clinical and epidemiological grounds, a throat swab for Mycoplasma pneumoniae PCR is recommended. [D]

- Serology with the complement fixation test and a range of other assays is widely available, although considerable caution is required in interpretation of results. [C]

\subsubsection{Tests for Chlamydophila species}

Culture

It is not appropriate for routine diagnostic laboratories to attempt culture of Chlamydophila from respiratory samples from patients with CAP as special laboratory precautions are required. (C psittaci is a "category 3 pathogen" indicating a high-risk pathogen that may put laboratory staff at risk of serious illness if infected occupationally.) C pneumoniae is very difficult to grow in the laboratory-culture is slow, timeconsuming, expensive and insensitive.

\section{Antigen detection}

Chlamydophila antigen can be detected in respiratory samples using DIF with species- and genus-specific monoclonal antibodies. ${ }^{234}$ [II] Species-specific reagents are not available for $C$ psittaci, which is antigenically highly diverse. DIF requires expertise in slide preparation and reading, and is not widely available in diagnostic laboratories. C pneumoniae can also be detected by DIF on throat swabs, with a comparable sensitivity to sputum. ${ }^{235}$ However, antigen may be detected for several months after "acute" infection, making interpretation difficult.

Chlamydophila antigen can also be detected in respiratory samples by EIA with comparable sensitivity to PCR, ${ }^{236}$ but this approach requires further studies.

\section{Serology}

Various serological assays are used in the diagnosis of respiratory Chlamydophila infections. The CFT is available in some diagnostic serology laboratories. Micro-immunofluorescence (MIF) and whole-cell immunofluorescence (WHIF) are specialised reference tests. Several EIAs have been described, and at least one is commercially available in the UK. Each of these assays has advantages and disadvantages, and there are particular problems in the serological diagnosis of $C$ pneumoniae infections.

The CFT uses a genus-specific antigen and is relatively sensitive and specific for diagnosing psittacosis. However in adults, most infections with $C$ pneumoniae are re-infections and these generate only a weak or absent CFT response.

The MIF and WHIF tests require considerable experience to read and interpret. They can detect a species-specific response, although this may be delayed for 4-6 weeks, especially with $C$ 
pneumoniae re-infections. They may also miss $C$ psittaci infections, depending on the particular serovars included in the test, and there are conflicting reports regarding the accuracy of these tests in reliably distinguishing chlamydial species. ${ }^{237}$ [IVb]

A commercial EIA has been used with success, ${ }^{238}\left[{ }^{[I I]}\right.$ but has not been shown to be significantly superior to CFT.

\section{Molecular techniques}

Amplification of Chlamydophila DNA by PCR using genus- or species-specific primers has been reported from a variety of respiratory samples. These molecular techniques for diagnosis of respiratory Chlamydophila infection are mostly confined to research/reference/specialist laboratories at the present time although, as with all molecular diagnostics, such tests are likely to become increasingly available, often in commercial kit formats.

\section{Recommendations}

- Chlamydophila antigen and/or PCR detection tests should be available for invasive respiratory samples from patients with high severity CAP or where there is a strong suspicion of psittacosis. [D]

- The complement fixation test remains the most suitable and practical serological assay for routine diagnosis of respiratory Chlamydophila infections. [B-] There is no currently available serological test that can reliably detect acute infection due to $C$ pneumoniae.

\subsubsection{PCR and serology for other respiratory pathogens}

PCR tests for a range of respiratory viruses and some atypical pathogens are becoming increasingly available through virology departments across the UK. The ready availability and extent of these investigations varies between individual laboratories and departments, although they are expected to increase over the foreseeable future. Where available, PCR tests are an extremely useful addition to the diagnostic armamentarium and have the advantage of being rapid (relevant on occasions for both clinical and infection control purposes) and sensitive, and so are to be preferred over serological tests. Evidence of influenza and/or other respiratory virus infections as a cause of primary viral pneumonia or as a copathogen in bacterial CAP is best sought by PCR of nose and throat swabs submitted in virus transport medium.

At present there are no readily available alternatives to serology for some of the atypical pathogens and, according to clinical and epidemiological parameters, serological investigations may be appropriate.

Respiratory serology usually comprises antibody tests for the atypical pathogens ( $M$ pneumoniae, Chlamydophila spp, $C$ burnetii), influenza A virus, influenza B virus, adenovirus, respiratory syncytial virus and $L$ pneumophila. Fewer laboratories rely on CFTs, which are time consuming and inconvenient to perform and have poor sensitivity and specificity.

There is little value in testing single serum samples taken within 7 days of the onset of CAP. Such samples can be stored until the follow-up (convalescent) sample is taken (7-10 days later) and the paired samples tested in parallel.

However, raised antibody titres-particularly to $L$ pneumophila or $M$ pneumoniae - may be found in some patients on or soon after hospital admission, particularly if the onset of symptoms is more than 7 days prior to admission. It is thus important that the date of onset of symptoms is clearly indicated on all serological request forms so that serum samples taken more than 1 week into the illness can be tested immediately.

A suggested algorithm for performing serological investigations is shown in fig 6.

\section{Recommendations}

- Where PCR for respiratory viruses and atypical pathogens is readily available or obtainable locally, this is preferred to serological investigations. [D]

- Where available, paired serology tests can be considered for patients with high severity CAP where no particular microbiological diagnosis has been made by other means (eg, culture, urine antigen, PCR) and who fail to improve, and/or where there are particular epidemiological risk factors. [D] The date of onset of symptoms should be clearly indicated on all serological request forms. [D]

- Serological tests may be extended to all patients admitted to hospital with CAP during outbreaks and when needed for the purposes of surveillance. The criteria for performing serology tests in these circumstances should be agreed locally between clinicians, laboratories and public health. [D]

\section{SECTION 6 SEVERITY ASSESSMENT}

\subsection{Why is severity assessment important?}

CAP presents to physicians both in primary and secondary care as a wide spectrum of illness from mild and self-limiting to lifethreatening and occasionally fatal disease. This breadth of illness severity is reflected in the variable mortality rates reported by studies of CAP in different clinical settings.

The decision regarding the most appropriate site of care, including whether hospitalisation of a patient with CAP is warranted, is the first and single most important decision in the overall management of CAP. It has consequences both on the level of treatment received by the patient as well as the overall costs of treatment. ${ }^{30}$ [III] This decision is best informed by an accurate assessment of the severity of illness at presentation and the likely prognosis. The recognition of patients at low risk of complications-and therefore suitable for treatment out of hospital - has the potential to reduce inappropriate hospitalisation and consequently inherent morbidity and costs.

When hospital admission is required, further management is also influenced by illness severity. This includes the extent of microbiological investigation, the choice of initial empirical antimicrobial agents, route of administration, duration of treatment and level of nursing and medical care. Early identification of patients at high risk of death allows initiation of appropriate antibiotic therapy and admission to an intensive care setting where assisted ventilation can be readily initiated if necessary.

6.2 What clinical factors and investigations are associated with a poor prognosis on univariate and multivariate analysis?

A large number of studies conducted in hospital and intensive care settings have employed univariate analysis to identify risk factors associated with a poor prognosis. In-hospital mortality has been the most common outcome measure. Some studies have used admission to the ICU as the main outcome measure. ${ }^{239}[\mathrm{Ib}]$ However, differences in ICU admission criteria make it difficult to compare results from these studies. This is 


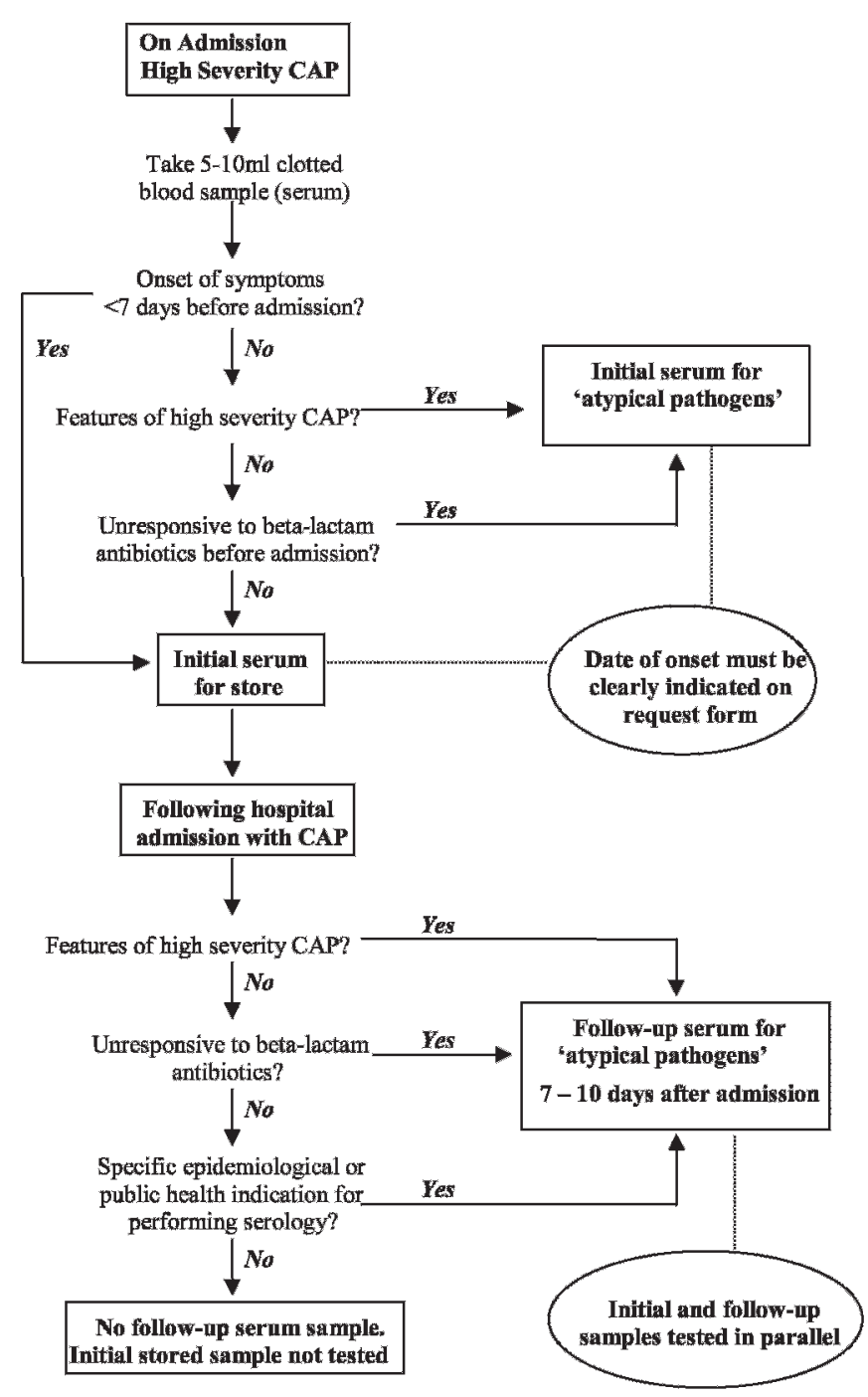

Figure 6 Suggested algorithm for serological testing for patients with high severity community acquired pneumonia (CAP).

reflected in the widely varying rates of admission of patients with CAP to ICU reported, ranging from 1-3\% in New Zealand to $5 \%$ in the UK, $12-18 \%$ in the USA and $35 \%$ in Germany. ${ }^{5}$ 239-242 [Ib] [Ib] [III] [II] [Ib] [III] In this document we have concentrated only on studies that have used mortality as the main outcome measure.

Univariate studies have suggested that over 40 different parameters are associated with mortality. An independent association of only a few of these risk factors with mortality has been consistently demonstrated by studies employing multivariate analysis. In particular, the following features are associated with an increased risk of mortality: increasing age $;^{5} 6241243-247[\mathrm{Ib}][\mathrm{Ib}][\mathrm{Ib}][\mathrm{Ia}][\mathrm{Ib}][\mathrm{Ib}][\mathrm{III}][\mathrm{Ib}]$ the presence of co-existing illnesses; 5 243-245 247-251 [Ib] [III] [Ib] [Ib] [Ib] [III] [II] [III] [III] raised respiratory

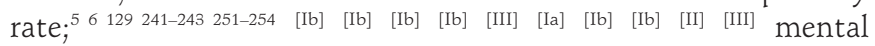
confusion; ; 129244246253255 [Ib] $[\mathrm{Ib}]$ [III] $\quad$ [III] $\quad$ [II] $\quad$ [Ib] low blood pressure; ; 69127129241243246249251253256257 [Ib] [II] [III] [Ib] [Ib] [Ia] [III] [II] [III] [II] [II] [Ib] hypoxaemia; 33241 [II] [Ib] respiratory failure; 5344258 [Ib] [II] [II] [Ib] high or low white cell count; ${ }^{6243}$ [Ib] [Ia] bilateral or progressive chest radiograph changes; ${ }^{5} 33129187243253256258259$ [Ib] [II] [Ib] [III] $[\mathrm{Ia}] \quad[\mathrm{II}] \quad[\mathrm{II}] \quad[\mathrm{III}, \mathrm{Ib}] \quad[\mathrm{Ib}]$ and a positive blood culture. $^{5} 333644243250258260$ [Ib] [II] [II] [II] [Ia] [III] [Ib] [III]
The lack of pyrexia, which is easily overlooked in clinical practice, has been identified as an important poor prognostic factor in some studies of elderly patients. ${ }^{69} 129$ 253 [II] [Ib] [II] Being previously bed-ridden and having been admitted from a nursing home are other poor prognostic factors that may be interrelated. ${ }^{5} 129153247$ [Ib] [Ib] [II] [Ib]

\subsubsection{Biomarkers and inflammatory markers}

A number of biomarker tests have been demonstrated on univariate and multivariate analyses to be independent prognostic factors for either 30-day or in-hospital mortality. These include procalcitonin, ${ }^{261-265}[\mathrm{II}][\mathrm{II}] \quad[\mathrm{Ib}][\mathrm{Ib}] \quad[\mathrm{II}]$ triggering receptor expressed on myeloid cells-1 (TREM-1), ${ }^{266[\mathrm{Ib}]}$ CD14, ${ }^{267}[\mathrm{Ib}]$ proadrenomedullin, ${ }^{268}[\mathrm{Ib}] \mathrm{CRP},{ }^{257} 269$ [Ib] [II] pro-atrial natriuretic peptide $^{270} 271[\mathrm{Ib}][\mathrm{III}]$ and pro-vasopressin. ${ }^{270}[\mathrm{Ib}]$ Further validation studies are required in most instances. Furthermore, determination of the majority of these biomarkers is not widely or routinely available at present.

Of the commonly available tests, one study has reported an association of a low CRP level of $<100 \mathrm{mg} / \mathrm{l}$ at the time of hospital admission with reduced risk for 30-day mortality, need for mechanical ventilation and/or inotropic support, and complicated pneumonia. ${ }^{257[\mathrm{Ib}]}$ This observation warrants further evaluation as a number of other studies have not found an association of admission CRP level with prognosis. ${ }^{261} 264265272$ [II] [Ib] [II] [Ib]

The level of D-dimers on admission has also been reported to be an independent prognostic marker. ${ }^{273} 274$ [Ib] [II] However, the clinical value of $\mathrm{D}$-dimers over and above a clinical predictive model has not been established. Moreover, since D-dimers are mainly used in the diagnostic investigation of patients with suspected venous thromboembolism, introducing D-dimers solely as a prognostic marker in CAP will potentially lead to increased diagnostic uncertainty or confusion which, in turn, may result in delayed diagnosis or inappropriate management.

\subsection{What predictive models for assessing severity on or shortly after hospital admission have been tested?}

Clinical assessment of disease severity is dependent on the experience of the attending clinician, but such clinical judgement has been shown to result in apparent underestimation of severity. ${ }^{241}[\mathrm{Ib}]$ No single prognostic factor of mortality is adequately specific and sensitive, so various severity scoring systems and predictive models have been developed in an attempt to help the clinician identify patients with pneumonia and a poor prognosis at an early stage.

However, predictive models have recognised limitations. First, no predictive model enables the unequivocal categorisation of patients into definite risk groups, and it is unrealistic to expect this. ${ }^{275} 276[\mathrm{IVb}][\mathrm{IVb}]$ A predictive model is therefore not a substitute for clinical judgement, but should be considered as an adjunct to clinical judgement. Second, the value of severity-based practice guidelines in improving clinical outcomes has not yet been fully established. ${ }^{103} 277278$ [III] [II] [II] Data demonstrating that severitybased practice guidelines are useful in increasing the proportion of patients managed as ambulatory outpatients without increasing morbidity or mortality have come from a cluster randomised trial, two large prospective observational studies

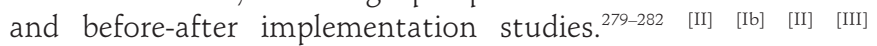
Whether these benefits were mainly due to the use of a predictive model and therefore better stratification of patients to different management strategies, or simply due to better implementation of practice guidelines for the management of 
$\mathrm{CAP}^{283}[\mathrm{II}]$ is difficult to differentiate. These encouraging results need confirmation in a wider setting, including the UK.

\section{Conclusions regarding predictive models}

- No predictive model allows the unequivocal categorisation of patients into definite risk groups.

- Predictive models based on severity are best viewed as useful adjuncts to clinical judgement.

- Regular reassessment of severity during the course of hospital stay is mandatory if treatment is to be adjusted appropriately, avoiding the morbidity of overtreatment as well as the complications of undertreatment.

\subsubsection{Pneumonia-specific predictive models Pneumonia Severity Index (PSI)}

The most widely studied predictive model in the management of CAP is the PSI developed in the USA. ${ }^{251}[\mathrm{Ib}]$ The PSI is based on 20 variables that are used to derive a score which enables patients to be stratified into five risk categories based on 30-day mortality. It was developed to identify patients at low risk of mortality who might be suitable for ambulatory outpatient care, and it is in this setting that it is best validated. In the original study, mortality rates were $0.1 \%, 0.6 \%, 0.9 \%, 9.3 \%$ and $27 \%$ for risk classes I, II, III, IV and V, respectively. The PSI has been studied in over 50000 patients worldwide and performs well. Patients in risk classes I-III are usually at low risk of mortality and therefore suitable for outpatient management. Due to the emphasis on age in the PSI, underestimation of severe pneumonia occasionally results. One study reported that up to $40 \%$ of patients with CAP who were hospitalised were assigned to low risk classes based on the PSI, ${ }^{284}[\mathrm{II}]$ and another reported that ICU admission occurred in $27 \%$ of patients assigned to PSI risk classes I-III. ${ }^{285}$ [II] These reports reflect the importance of clinical judgment in assessing disease severity.

In clinical practice, the major limitation of the PSI with regard to its widespread and routine adoption in primary care, emergency departments or medical admission units is the complexity involved in the calculation of the score.

\section{CURB65 score}

The CURB65 score was developed based on a study of over 1000 prospectively studied patients with CAP from three countries: the UK, New Zealand and the Netherlands. ${ }^{255}[\mathrm{lb}]$ The 6-point CURB65 score, one point for each of Confusion, Urea > $7 \mathrm{mmol} / \mathrm{l}$, Respiratory rate $\geqslant 30 / \mathrm{min}$, low systolic $(<90 \mathrm{~mm} \mathrm{Hg})$ or diastolic ( $\leqslant 60 \mathrm{~mm} \mathrm{Hg}$ ) Blood pressure, age $\geqslant 65$ years (CURB65 score) based on information available at initial hospital assessment, enabled patients to be stratified according to increasing risk of mortality (score $0,0.7 \%$; score 1 , $2.1 \%$; score $2,9.2 \%$; scores $3-5,15-40 \%$ ). A similar pattern of increasing disease severity was reported when only clinical parameters were considered (CRB65) giving a 5-point score (risk of mortality for each score: score $0,1.2 \%$; score $1,5.3 \%$; score 2 , $12.2 \%$; scores $3-4$, up to $33 \%$ ).

Since then, the CURB65 score has been studied in over 12000 patients. ${ }^{257} 266$ 286-295 [Ib] [Ib] [II] [Ib] [Ib] [II] [Ib] [II] [III] [II] [Ib] [III] The results from all but one of the prospective validation studies indicate that the PSI and the CURB65 score perform equally well at discriminating patients into mortality risk groups. The CRB65 score has been specifically studied in over 6000 patients representing a mix of patients seen both in the community and in hospitals. ${ }^{255} 286287289$ 296-299 [Ib] [II] [Ib] [II] [Ib] [II] [Ib] [II] All studies reported findings similar to the derivation study and, in certain studies, the CRB65 score was reported to be of similar discriminatory value to the CURB65 score. ${ }^{286} 298$ [II] $[\mathrm{Ib}]$ In prospective studies reporting from the UK, $41-45 \%$ of patients hospitalised with CAP had CURB65 scores of 0-1, 25-28\% had a CURB65 score of 2 and 29-34\% had CURB65 scores of 3 or more. ${ }^{255} 257$

Other predictive models have been developed, including models to predict admission to intensive care as the primary outcome, or to predict a composite outcome comprising either mortality or need for intensive care. ${ }^{246} 287291294$ 300-303 [III] [Ib] [Ib] [Ib] [II] [III] [Ib] [Ib] Widespread validation of most of these models in different settings or patient cohorts has not yet occurred. Differences between studies in relation to criteria for intensive care admission need to be taken into account when interpreting these data.

\subsubsection{Generic predictive models}

Generic predictive models such as the sepsis score or APACHE II scoring system have also been tested in patients with CAP and

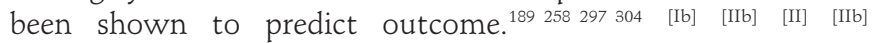
However, general adoption of scoring systems originally designed for use within an intensive care setting is difficult, time-consuming and likely to be impractical. In addition, pneumonia-specific predictive models have been shown to outperform generic predictive models such as a modified Early Warning Score, in the context of CAP. ${ }^{248} 289$ [III] [II]

\subsection{What severity assessment strategy is recommended for CAP?}

We have been keen to recommend one severity assessment strategy that is applicable to adults of all ages, simple to remember and practical to implement both in the community and in hospital.

With these principles in mind, the CURB65 score in conjunction with clinical judgement is recommended as the initial severity assessment strategy in hospitals for CAP. The evidence base for the CURB65 score is robust and continues to increase, adding to the strength of the current recommendation. The simplified CRB65 score which only relies on clinical factors in conjunction with clinical judgement is recommended as the severity assessment strategy in community or primary care settings for CAP.

\section{Summary of the CURB65 score}

- Confusion: New mental confusion, defined as an Abbreviated Mental Test score ${ }^{191}$ [II] (summarised in box 2) of 8 or less.

- Urea: Raised $>7 \mathrm{mmol} / \mathrm{l}$ (for patients being seen in hospital).

- Respiratory rate: Raised $\geqslant 30 /$ min.

- Blood pressure: Low blood pressure (systolic <90 mm Hg and/or diastolic $\leqslant 60 \mathrm{~mm} \mathrm{Hg}$ ).

- Age $\geqslant 65$ years.

\subsubsection{Clinical judgement}

Clinical judgement is essential when deciding on the management of all patients with CAP, particularly in the following three areas with regard to severity assessment:

- Interpretation of CURB65 score. Each patient must be managed individually and the interpretation of the CURB65 score is best refined through clinical judgement that takes into account all the clinical information available at the time. For instance, the combination of age $<50$ years, the 
absence of coexisting disease and a CRB65 or CURB65 score of 0 identifies patients with a very good prognosis who should be suitable for home treatment. In comparison, young patients with a respiratory rate of $>40 /$ min may warrant hospital supervised management despite a CRB65 or CURB65 score of 1 . Clinical judgement is especially important in patients at high risk of death (CURB65 scores 3,4 and 5) in whom decisions regarding intravenous administration of antibiotics or transfer to critical care facilities need to be made.

- Stability of comorbid illnesses. The CURB65 score is primarily a CAP severity assessment tool. Pneumonia may result in a worsening of comorbid illness that warrants hospital or critical care management irrespective of severity of pneumonia.

- Social circumstances. The morbidity associated with CAP negatively influences the functional status of patients and therefore the extent that patients may be able to manage at home within a given set of social circumstances. Patient choice should also be considered when determining social circumstances and the feasibility of further management at home.

\section{Recommendations}

- Clinical judgement is essential in disease severity assessment. [D]

- The stability of any comorbid illness and a patient's social circumstances should be considered when assessing disease severity. [D]

\subsection{Severity assessment of CAP in patients seen in the community}

The severity assessment of CAP in patients seen in the community is shown in fig 7.

\section{Recommendations}

- For all patients, clinical judgement supported by the CRB65 score should be applied when deciding whether to treat at home or refer to hospital. [D]

- Patients who have a CRB65 score of 0 are at low risk of death and do not normally require hospitalisation for clinical reasons. $[\mathrm{B}+]$

- Patients who have a CRB65 score of 1 or 2 are at increased risk of death, particularly with a score of 2 ,

\section{Box 2 Abbreviated Mental Test}

The Abbreviated Mental Test (each question scores 1 mark, total 10 marks)

- Age

- Date of birth

- Time (to nearest hour)

- Year

- Hospital name

- Recognition of two persons (eg, doctor, nurse)

- Recall address (eg, 42 West Street)

- Date of First World War

- Name of monarchs

- Count backwards $20 \rightarrow 1$

A score of 8 or less has been used to define mental confusion in the CURB65 severity score. and hospital referral and assessment should be considered. $[\mathrm{B}+]$

- Patients who have a CRB65 score of 3 or more are at high risk of death and require urgent hospital admission. $[\mathrm{B}+]$

-When deciding on home treatment, the patient's social circumstances and wishes must be taken into account in all instances. [D]

\subsection{Severity assessment of CAP in patients seen in hospital}

The severity assessment of CAP in patients seen in hospital is shown in fig 8.

\section{Recommendations}

- For all patients, the CURB65 score should be interpreted in conjunction with clinical judgement. [D]

- Patients who have a CURB65 score of 3 or more are at high risk of death. These patients should be reviewed by a senior physician at the earliest opportunity to refine disease severity assessment and should usually be managed as having high severity pneumonia. Patients with CURB65 scores of 4 and 5 should be assessed with specific consideration to the need for transfer to a critical care unit (high dependency unit or intensive care unit). [B+]

- Patients who have a CURB65 score of 2 are at moderate risk of death. They should be considered for short-stay inpatient treatment or hospital-supervised outpatient treatment. $[\mathrm{B}+]$

- Patients who have a CURB65 score of 0 or 1 are at low risk of death. These patients may be suitable for treatment at home. $[\mathrm{B}+]$

- When deciding on home treatment, the patient's social circumstances and wishes must be taken into account in all instances. [D]

\subsection{Reviewing severity status after initial assessment in hospital} Summary

- Regular and structured clinical review and reassessment of disease severity facilitates the stepping down and stepping up of antibiotic management. [Ib]

\section{Recommendations}

- Regular assessment of disease severity is recommended for all patients following hospital admission. The "post take" round by a senior doctor and the medical team provides one early opportunity for this review. [D]

- All patients deemed at high risk of death on admission to hospital should be reviewed medically at least 12hourly until shown to be improving. [D]

\section{SECTION 7 GENERAL MANAGEMENT IN THE COMMUNITY AND IN HOSPITAL}

7.1 What general management strategy should be offered to patients treated in the community?

Patients with CAP may present with fever, cough, sputum production or pleuritic pain and usually have localised signs on chest examination. They should be advised to rest and avoid smoking ${ }^{305}[\mathrm{IIb}]$ and, especially when febrile, be encouraged to drink plenty of fluids. It is important to relieve pleuritic pain using simple analgesia such as paracetamol or non-steroidal 


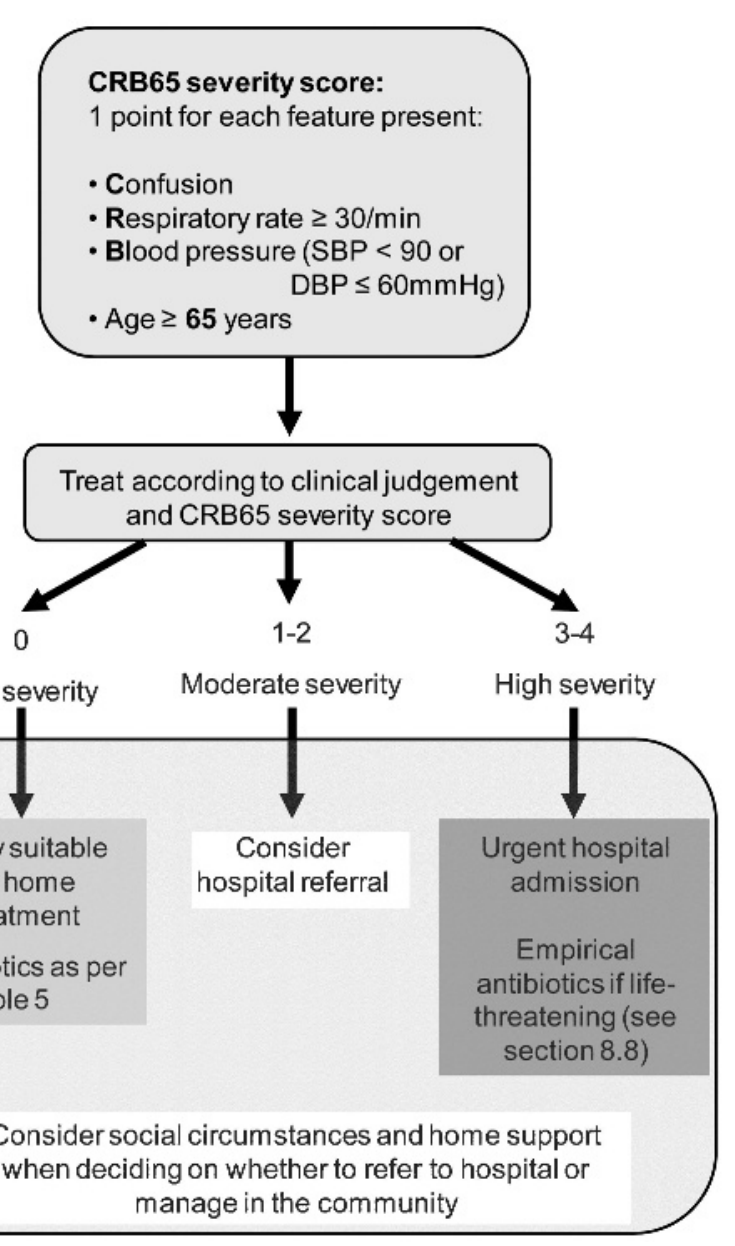

Figure 7 Severity assessment of community acquired pneumonia (CAP) in patients seen in the community (CRB65 severity score plus clinical judgement). DBP, diastolic blood pressure; SBP, systolic blood pressure.

anti-inflammatory drugs. Physiotherapy is of no proven benefit in acute pneumonia. ${ }^{306}$ [III] Nutritional status appears important both to the outcome and the risk of acquiring pneumonia and, in prolonged illness, nutritional supplements may be helpful. Patients with pneumonia are often catabolic and those aged $>55$ years who are malnourished appear to be at greater risk of developing pneumonia. ${ }^{150} 307$ [III] [III]

Patients with pneumonia often become hypoxic because pulmonary blood flow takes place through unventilated lung tissue. The clinical signs of hypoxia are non-specific and often difficult to recognise in the early stages. They include altered mental state, dyspnoea and tachypnoea. Respiratory rate should therefore always be assessed. Central cyanosis is unreliable both as a clinical sign and also as an indicator of tissue hypoxia. In contrast, pulse oximetry which measures arterial oxygen saturation $\left(\mathrm{SpO}_{2}\right)$ is, in most situations, a simple and reliable method of assessing oxygenation. However, poor peripheral perfusion, jaundice and pigmented skin can produce a falsely low saturation and carboxyhaemoglobin a falsely high saturation. Pulse oximetry is now widely available in North America. A survey of 944 outpatients and 1332 inpatients with evidence of CAP enrolled from five sites in the USA and Canada reported increasing assessment of arterial oxygen saturation with pulse oximetry in up to $58 \%$ of outpatients and $85 \%$ of inpatients. ${ }^{308}$ [II] It is recommended that pulse oximetry, with appropriate training, should become more widely available in general practice for use in the assessment of patients who may have pneumonia and other acute respiratory illnesses. Oxygen saturation below $94 \%$ in a patient with CAP is an adverse prognostic feature and also an indication for oxygen therapy, ${ }^{309}$ [IVb] which will usually require urgent referral to hospital.

Patients who fall outside the low severity criteria for CAP should be assessed for the need for hospital referral (see Section 6). Social factors will also play an important part in the decision to refer a patient to hospital. Patients with moderate or high severity pneumonia should be admitted to hospital and managed, where possible, with input from a physician with an interest in respiratory medicine.

\section{Recommendations}

- Patients with suspected CAP should be advised to rest, to drink plenty of fluids and not to smoke. [D]

- Pleuritic pain should be relieved using simple analgesia such as paracetamol. [D]

- The need for hospital referral should be assessed using the criteria recommended in section 6 . [C]

- Pulse oximetry, with appropriate training, should be available to general practitioners and others responsible for the assessment of patients in the out-of-hours setting for the assessment of severity and oxygen requirement in patients with CAP and other acute respiratory illnesses. [D]

\subsection{What review policy should be adopted in patients managed} in the community?

When to review a patient with CAP in the community will be determined by the initial severity assessment and other factors such as reliable help in the home. Patients assessed as being at low severity should improve on appropriate therapy within $48 \mathrm{~h}$, at which time severity reassessment is recommended. Those who fail to improve within $48 \mathrm{~h}$ should be considered for hospital admission. Patients who do not fulfil the criteria for low severity and are being managed at home will require more frequent review.

\section{Recommendations}

- Review of patients in the community with CAP is recommended after $48 \mathrm{~h}$ or earlier if clinically indicated. Disease severity assessment should form part of the clinical review. [D]

- Those who fail to improve after $48 \mathrm{~h}$ of treatment should be considered for hospital admission or chest radiography. [D]

\subsection{What general management strategy should be offered to patients in hospital?}

Initial management

There is some evidence that use of a critical care pathway for patients referred to hospital can reduce the hospital admission of low-risk patients and can also rationalise inpatient management. $^{310}[\mathrm{lb}]$

All patients referred to hospital with CAP should have a chest radiograph (if not already performed in the community) and should have oxygenation assessed by pulse oximetry, preferably while breathing air. Those with $\mathrm{SpO}_{2}<94 \%$ should have arterial blood gas measurements, as should all patients with features of high severity pneumonia. Knowledge of the inspired oxygen concentration is essential to the interpretation of blood gas 
Figure 8 Hospital management of community acquired pneumonia (CAP) in the first $4 \mathrm{~h}$. CXR, chest $x$ ray; DBP, diastolic blood pressure; SBP, systolic blood pressure.

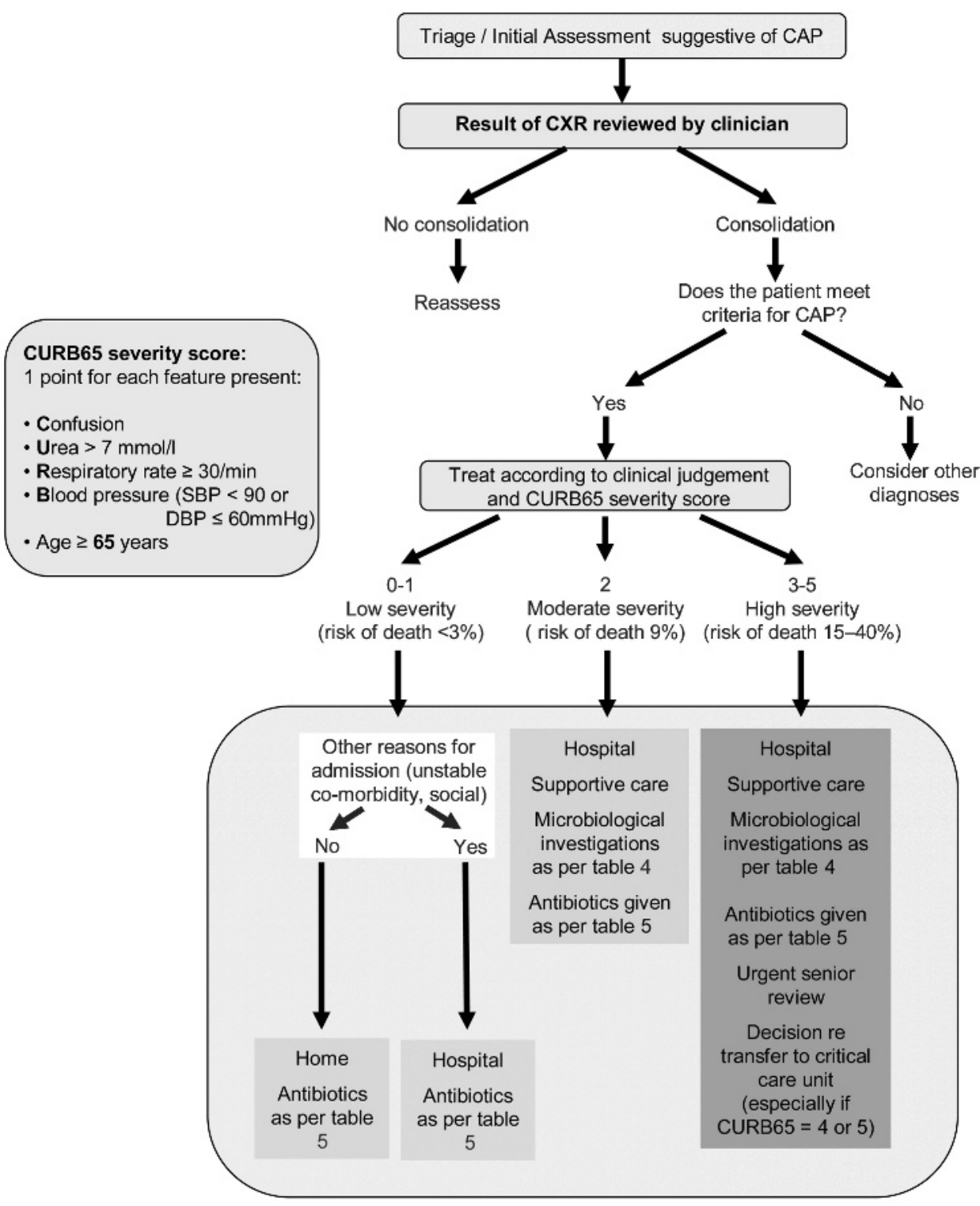

Aim by 4 hours: diagnosis made and management including antibiotics started

measurements and should be clearly recorded with the blood gas result.

Continuous oxygen therapy is usually indicated for those patients with arterial oxygen tension $\left(\mathrm{PaO}_{2}\right)<8 \mathrm{kPa} .{ }^{183}$ The aim of oxygen therapy should be to maintain $\mathrm{PaO}_{2} \geqslant 8 \mathrm{kPa}$ or $\mathrm{SpO}_{2}$ 94-98\% in patients who are not at risk of hypercapnic respiratory failure. In nearly all cases of CAP, unless complicated by severe COPD with ventilatory failure, high concentrations of oxygen of $35 \%$ or more are indicated and can be safely used.

High concentration oxygen therapy given to patients at risk of hypercapnic respiratory failure, such as patients with moderate or severe COPD, can reduce hypoxic drive and increase ventilation-perfusion mismatching. In such patients, initial treatment should be with low oxygen concentrations (24-28\%), guided by arterial blood gas measurements, with the aim of keeping oxygen saturation at $88-92 \%$ without causing a fall in arterial $\mathrm{pH}\left(\mathrm{pH}<7.35\right.$ or $\left[\mathrm{H}^{+}\right]>45 \mathrm{nmol} / 1 .{ }^{183}$ In selected cases, non-invasive ventilation or respiratory stimulants may be of value and transfer to a high dependency unit or ICU area should be considered. If the patient is hypercapnic $\left(\mathrm{PaCO}_{2}\right.$
$>6 \mathrm{kPa}$ or $45 \mathrm{~mm} \mathrm{Hg}$ ) and acidotic $\left(\mathrm{pH}<7.35\right.$ or $\left[\mathrm{H}^{+}\right]>$ $45 \mathrm{nmol} / \mathrm{l}$ ), ventilatory support should be considered, especially if acidosis has persisted for more than $30 \mathrm{~min}$ despite appropriate therapy.

Patients admitted with pneumonia should be assessed for volume depletion and may require intravenous fluids.

The use of unfractionated or low molecular weight heparins for the prevention of venous thromboembolism in patients with respiratory tract infections has been demonstrated in clinical trials. ${ }^{311-313}$ [Ia]

A randomised study $(n=458)$ found early mobilisation (sitting out of bed for at least 20 min within the first $24 \mathrm{~h}$ of hospital admission with increasing mobility on subsequent days) decreased length of stay by 1.1 days. ${ }^{314}$ [Ib] Further studies are needed.

Routine airway clearance has not been shown to improve outcome. $^{315[\mathrm{Ib}]}$

While chest physiotherapy is of no proven value in acute pneumonia, ${ }^{315}$ [III] a single randomised trial using positive expiratory pressure has suggested that "bottle-blowing" into 
an underwater seal bottle on 10 occasions daily can shorten hospital stay. ${ }^{316}[\mathrm{II}]$

In cases of severe pneumonia requiring prolonged hospital admission, increased nutritional support (whether enteral, parenteral or via nasogastric feeding) should be arranged.

\section{Recommendations}

- All patients should receive appropriate oxygen therapy with monitoring of oxygen saturations and inspired oxygen concentration with the aim to maintain $\mathrm{PaO}_{2}$ at $\geqslant 8 \mathrm{kPa}$ and $\mathrm{SpO}_{2}$ 94-98\%. High concentrations of oxygen can safely be given in patients who are not at risk of hypercapnic respiratory failure. [D]

- Oxygen therapy in patients at risk of hypercapnic respiratory failure complicated by ventilatory failure should be guided by repeated arterial blood gas measurements. [C]

- Patients should be assessed for volume depletion and may require intravenous fluids. [C]

- Prophylaxis of venous thromboembolism with low molecular weight heparins should be considered for all patients who are not fully mobile. $[\mathrm{A}+]$

- Nutritional support should be given in prolonged illness. [C]

- Medical condition permitting, patients admitted to hospital with uncomplicated CAP should sit out of bed for at least $20 \mathrm{~min}$ within the first $24 \mathrm{~h}$ and increase mobility each subsequent day of hospitalisation. [A-]

- Patients admitted with uncomplicated pneumonia should not be treated with traditional airway clearance techniques routinely. $[\mathrm{B}+]$

- Patients should be offered advice regarding expectoration if there is sputum present. [D]

- Airway clearance techniques should be considered if the patient has sputum and difficulty with expectoration or in the event of a pre-existing lung condition. [D]

\section{Monitoring in hospital}

Pulse, blood pressure, respiratory rate, temperature, oxygen saturation (with a recording of the inspired oxygen concentration at the same time) and mental status should be measured initially at least twice daily. Those with high severity pneumonia, requiring continuous oxygen or cardiovascular support, should be monitored more frequently. Vital signs may be captured by an early warning score and can be used to trigger escalation or de-escalation of management. ${ }^{317}$

The acute phase reactant CRP is a sensitive marker of progress in pneumonia. ${ }^{187} 189$ [III] $[\mathrm{III}]$ Prospective studies have shown that repeat measurement of CRP at day 3 or 4 is helpful in identifying patients with treatment failure. ${ }^{257} 318$ [ib] A failure of CRP to fall by $50 \%$ is associated with increased 30-day mortality, increased need for mechanical ventilation and/or inotropic support and increased incidence of complicated pneumonia such as empyema (see Section 6.2.1 and Section 9). $257[\mathrm{Ib}]$

Failure to improve over 4 days is an indication to repeat the chest radiograph.

In discharge planning, a US prospective multicentre observational cohort study of 680 patients admitted to hospital with CAP reported that almost $20 \%$ left hospital with one or more unstable factors in the $24 \mathrm{~h}$ prior to discharge. These included temperature $>37.8^{\circ} \mathrm{C}$, heart rate $>100 / \mathrm{min}$, respiratory rate $>24 / \mathrm{min}$, systolic blood pressure $<90 \mathrm{~mm} \mathrm{Hg}$, oxygen saturation $<90 \%$, inability to take oral medication or abnormal mental status. Forty-six per cent of those discharged home with two of these unstable factors died or were readmitted within 30 days. In contrast, only $11 \%$ of those with no unstable factors died or were readmitted within 30 days. ${ }^{319}$ [II] A similar prospective cohort study in 373 patients found that $22 \%$ of patients were discharged with one or more unstable factors, which led to increased mortality $(14.6 \%$ vs $2.1 \%)$ and increased readmission within 60 days. $^{320}[\mathrm{II}]$

\section{Recommendations}

- Temperature, respiratory rate, pulse, blood pressure, mental status, oxygen saturation and inspired oxygen concentration should be monitored and recorded initially at least twice daily and more frequently in those with severe pneumonia or requiring regular oxygen therapy. [C]

- C-reactive protein should be remeasured and a chest radiograph repeated in patients who are not progressing satisfactorily after 3 days of treatment. [B+]

- Patients should be reviewed within $24 \mathrm{~h}$ of planned discharge home, and those suitable for discharge should not have more than one of the following characteristics present (unless they represent the usual baseline status for that patient): temperature $>37.8^{\circ} \mathrm{C}$, heart rate $>100 / \mathrm{min}$, respiratory rate $>24 / \mathrm{min}$, systolic blood pressure $<90 \mathrm{~mm} \mathrm{Hg}$, oxygen saturation $<90 \%$, inability to maintain oral intake and abnormal mental status. $[\mathrm{B}+]$

\subsection{What advice should be given regarding the critical care management of CAP?}

Severity assessment is an important part of hospital management as it can identify those patients at increased risk of death. Patients who fulfil the severity criteria for high severity CAP on admission and who do not respond rapidly should be considered for transfer to a high dependency unit or a critical care unit (see Section 6). Persisting hypoxia with $\mathrm{PaO}_{2}<8 \mathrm{kPa}$ despite maximal oxygen administration, progressive hypercapnia, severe acidosis ( $\mathrm{pH}<7.26$ ), shock, or depressed consciousness are also indications for transfer to critical care for assisted ventilation and cardiovascular support..$^{321}[\mathrm{IVb}]$

Observational studies indicate that non-invasive ventilation (NIV) is being used to treat respiratory failure in CAP in many critical care units. ${ }^{322}$ [III] However, a systematic review in 2003 concluded that there was no clear benefit of NIV in acute respiratory failure in a non-COPD population. ${ }^{323}$ [Ia] A further systematic review published in 2004 drew broadly similar conclusions. This review included all randomised studies of patients with acute hypoxaemic respiratory failure given NIV. This was a very heterogeneous group and included immunosuppressed patients, those following lung resection and cases of post-extubation failure. Pooled data suggested a reduced need for intubation with NIV but no effect on mortality once studies including patients with COPD or pulmonary oedema were excluded. $^{324}$ [Ia] The number of patients with CAP included in these studies has been very limited; only one study exclusively focused on CAP and even this trial only recruited 33 patients without COPD. ${ }^{325}$ [II]

Continuous positive airways pressure (CPAP) is also used in some cases of pneumonia. However, a randomised controlled trial (RCT) of 123 patients with acute lung injury (61 with 
pneumonia) published in 2000 found no reduction in intubation or improved outcome with CPAP. ${ }^{326}$ [II] In addition, CPAP appeared to delay intubation in some patients with adverse consequences. In the non-COPD population with CAP, noninvasive modes of respiratory support are unlikely to be successful. This is particularly the case in patients developing other organ failure.

Despite the apparent benefit of activated protein C (APC) in patients with severe sepsis shown in the PROWESS study, ${ }^{327}$ [Ia] there is still substantial controversy about clinical efficacy. The importance of this issue to CAP is highlighted in a retrospective subgroup analysis of outcome in the subgroup of patients with CAP in the PROWESS study. ${ }^{328}$ [II] More than one-third of patients recruited were diagnosed as having severe CAP, although this did not correspond precisely with the Centre for Disease Control definition. Twenty-eight day mortality was reduced in the treatment group (odds ratio (OR) $0.81,95 \% \mathrm{CI}$ 0.67 to 0.93 ). By day 90 , the OR crossed the no-effect line. However, the groups were not well matched for important covariables and the improved outcome was only found after statistical adjustment. There was also some evidence that outcome in the less severe CAP group treated with APC was worse. A recent systematic review of APC in sepsis concluded that there was no definite evidence for efficacy of APC in sepsis, but the risk of bleeding was increased. ${ }^{329}$ [Ia] In view of the uncertainty, a further multicentre RCT of APC in severe sepsis is being conducted. APC continues to have a European product licence for the treatment of severe sepsis, including that caused by CAP. No definite treatment recommendation can be given until the results of the new RCT become available.

One RCT and a systematic review on steroids in severe CAP have been published since the last guidelines update. The single RCT enrolled 47 patients to placebo versus 7-day hydrocortisone infusion. ${ }^{330}$ [II] The trial was stopped early because of reported efficacy of the steroid treatment. However, the primary end point was gas exchange and mortality was very low in both groups. In addition, patients were not well matched (controls were sicker and the steroid group received more NIV). The systematic review identified three RCTs of steroids in CAP. ${ }^{331}{ }^{[\mathrm{Ia}]}$ All were small underpowered studies (including the above), which were not optimally conducted. The review concluded that there was no evidence for the use of steroids in severe CAP. In addition, a recent multicentre RCT of patients with severe sepsis found no survival benefit with hydrocortisone treatment. ${ }^{332}[\mathrm{lb}]$ This was a heterogeneous group, but a significant number had respiratory infections. There is therefore no high-grade evidence for the efficacy of steroids in severe CAP.

Granulocyte colony stimulating factor (G-CSF) has been the subject of a number of clinical trials in severe infection. A recent Cochrane review of 2018 patients with pneumonia revealed that G-CSF, as an adjunct to antibiotics, did not improve 28-day mortality. $^{333}$ [ia]

Bronchoscopy after intubation may be valuable to remove retained secretions, to obtain further samples for culture and to exclude endobronchial abnormality such as carcinoma. Hospital-acquired ventilator-associated pneumonia can occur in approximately $14 \%$ of patients mechanically ventilated for severe CAP and causes increased mortality. ${ }^{334}$ [III] Other aspects of ICU management are outside the scope of these guidelines.

\section{Recommendations}

- Patients with CAP admitted to ICUs should be managed by specialists with appropriate training in intensive care working in close collaboration with specialists in respiratory medicine. [D]

- Neither non-invasive ventilation (NIV) nor continuous positive airways pressure (CPAP) support is routinely indicated in the management of patients with respiratory failure due to CAP. [A-]

- If a trial of non-invasive support is considered indicated in CAP, it must only be conducted in a critical care area where immediate expertise is available to enable a rapid transition to invasive ventilation. [D]

- Steroids are not recommended in the routine treatment of high severity CAP. $[\mathrm{A}+]$

- Granulocyte colony stimulating factor is not routinely recommended as an adjunct to antibiotics. $[\mathrm{A}+]$

\subsection{What arrangements should be made for follow-up after hospital discharge and by whom?}

It is usual practice to arrange "routine" hospital clinic follow-up and repeat the chest radiograph at around 6 weeks after discharge. However, there is no evidence on which to base a recommendation regarding the value of this practice in patients who have otherwise recovered satisfactorily. It is also not known whether there is any value in arranging clinical followup in a hospital clinic rather than with the patient's general practitioner. The main concern is whether the CAP was a complication of an underlying condition such as lung cancer (see Section 5.6).

At discharge or at follow-up, patients should be offered access to information about CAP. In one study of 200 patients who had recently recovered from CAP, a patient information leaflet was judged to be very helpful by the majority of patients. ${ }^{335}$ [III] A patient information leaflet on CAP is available on request from British Lung Foundation Headquarters (British Lung Foundation, Freepost SW1233, London EC1B 1BR) and British Lung Foundation UK regional offices.

\section{Recommendations}

- Clinical review should be arranged for all patients at around 6 weeks, either with their general practitioner or in a hospital clinic. [D]

- At discharge or at follow-up, patients should be offered access to information about CAP such as a patient information leaflet. [D]

- It is the responsibility of the hospital team to arrange the follow-up plan with the patient and the general practitioner. [D]

Recommendations regarding whether or not to repeat the chest radiograph or perform further investigations at follow-up are given in Section 5.6.

\section{SECTION 8 ANTIBIOTIC MANAGEMENT}

\subsection{Introduction}

Antimicrobial chemotherapy is essential to the management of CAP. While mild pneumonia may be self-limiting, the timely use of appropriate antibiotics abbreviates illness, reduces the risk of complications and lowers mortality.

Few pneumonias are defined microbiologically at initial assessment and hence most prescribing is empirical, especially when managed in the community. Among hospitalised patients the aetiology may be determined, thereby permitting modification of the initial empirical regimen. However, in practice this applies to the minority of infections. ${ }^{194}\left[{ }^{[I]}\right.$ Clinical, epidemiological 
and radiographic information is rarely predictive of the microbial aetiology. An important principle is that pathogens responsible for CAP are diverse and vary in their ability to cause severe disease. $^{243}[\mathrm{lb}]$ The highest mortality rates are associated with pneumococcal infection and with Legionnaires' disease.

Severity assessment and the association of pre-existing comorbid disease is essential in predicting prognosis and, in turn, determines management, choice of antibiotic therapy and its method of administration (see Section 6).

\subsection{Antibiotic stewardship and the individual clinician's responsibility to prevent the overuse of antibiotics when managing CAP}

The choice of antibiotic regimen has consequences beyond the management of the individual patient. The inappropriate application of CAP guidelines to community acquired lower respiratory tract infections other than pneumonia and hospital acquired pneumonia leads, in turn, to inappropriate and potentially excessive antibiotic use. Too loose an interpretation of "severe pneumonia" also contributes to the overprescribing of macrolides and $\beta$-lactams, especially when administered parenterally, in the management of hospitalised patients with CAP.

Overuse of antibiotics in CAP increases the cost of management and, particularly in relation to quinolones and cephalosporins, serves as a driver for health care-associated infections, including MRSA and C difficile infection. ${ }^{18}$ 336-342 [Ib] [II] [IVa] [II] [II] [Ib] [Ia] [Ib] The avoidance of inappropriate or excessive use of antibiotics is specifically discussed in Section 8.18. Proper patient selection for treatment and the correct use of antimicrobial agents are emphasised in this document.

In particular, these guidelines stress that the use of empirical broad-spectrum antibiotics is initially recommended only in patients with high severity CAP. This group of patients comprise approximately one-third of all patients admitted to hospital with confirmed CAP. Regular review and the prompt "de-escalation" to narrow-spectrum antibiotics based on early microbiological investigations are also emphasised.

\subsection{Antibiotic resistance of respiratory pathogens}

The rate of increase in resistance among respiratory pathogens has tended to level off in recent years.

Antibiotic resistance among $S$ pneumoniae is the main concern owing to the dominance of this organism as a cause of CAP and because penicillin and macrolide resistance are frequently linked..$^{343} 344$ [II] [II] Resistance among pneumococci is the result of alterations in one or more of the penicillin-binding proteins, thus reducing their affinity for penicillin. This in turn leads to a requirement for higher drug concentrations to bring about death of the organism. Of the $>90$ known pneumococcal serotypes, a small number have been responsible for penicillin resistance worldwide, among which selected clones (eg, 23F, 9V and 6B) have become widely disseminated. However, despite these concerns, the clinical importance of in vitro penicillin resistance among $S$ pneumoniae remains uncertain when treating pneumo-

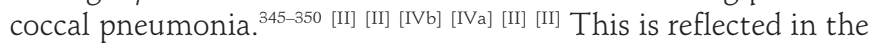
continued ability of current doses of penicillins to inhibit strains of intermediate susceptibility (minimum inhibitory concentration (MIC) of penicillin $0.1-1.0 \mathrm{mg} / \mathrm{l}$ ), as well as many strains exhibiting higher level resistance (as defined by an MIC $>1 \mathrm{mg} / \mathrm{l}$ ). The British Society of Antimicrobial Chemoptherapy (BSAC) surveillance project has reported on pneumococcal susceptibility to various antimicrobials from both blood and respiratory tract samples in the UK and Ireland 1999/2000 to 2006/7. They report no convincing evidence of an increase in non-susceptibility over time, $94 \%$ of bacteraemia isolates and $92 \%$ of respiratory isolate being fully susceptible to penicillin and $85 \%$ and $88 \%$ of blood and respiratory isolates, respectively, susceptible to erythromycin and, by implication, other macrolides. ${ }^{351}[\mathrm{II}]$ With regard to erythromycin, resistance is the result of genetic mutations that either affect the target site (erm gene mutations) or result in elimination of the drug by an efflux pump (mef gene mutation). The distribution of such strains differs internationally and probably explains variation in the clinical impact of such resistance, since erm gene mutations are linked to high level resistance. Tetracyclines are not widely used in the treatment of CAP and resistance among $S$ pneumoniae is relatively low. Likewise, reduced susceptibility of $S$ pneumoniae to fluoroquinolones is beginning to be reported, but generally remains low in the UK. ${ }^{352}[\mathrm{III}$ Recent trends in penicillin, erythromycin and tetracycline resistance of around 4000-5000 S pneumoniae isolated from blood and cerebrospinal fluid cultures each year in England and Wales and reported routinely to the Health Protection Agency (HPA) are shown in fig 9 (HPA unpublished data).

Beta-lactamase production among $H$ influenzae varies geographically but ranges from $2 \%$ to $17 \%$ in various parts of the UK. ${ }^{344}{ }^{353}[\mathrm{II}][\mathrm{II}]$ Data from the BSAC surveillance programme in the UK and Ireland $1999 / 2000$ to $2006 / 7$ suggest that $\beta$ lactamase production in $H$ influenzae has been relatively stable at around $15 \%$ over the study period. ${ }^{354}$ [II] However, this is an uncommon cause of pneumonia and, unless local data suggest otherwise, there is insufficient justification to include a $\beta$ lactamase resistant antibiotic regimen in initial empirical therapy of low or moderate severity CAP. $M$ catarrhalis is an even rarer cause of CAP, for which the same argument applies.

$S$ aureus is widely resistant to penicillin, ${ }^{355}$ [II] and an increasing number are now methicillin-resistant (MRSA). When occurring in the community within the UK, this generally reflects hospitalisation within the recent past or residence within a nursing home. Hence, $\beta$-lactamase unstable penicillins (penicillin G, aminopenicillins) and, in the case of MRSA, isoxazolyl penicillins (flucloxacillin, cloxacillin) and cephalosporins are inappropriate for such infections.

L pneumophila and Legionella spp in general remain susceptible to fluoroquinolones, macrolides and rifampicin, although in vitro low level resistance has been found in some isolates. However, the clinical significance of these observations remains unclear. ${ }^{356}$

\subsection{Newer antibiotics}

Since the 2001 guidelines were published, moxifloxacin has been licensed in the UK for the treatment of "non-severe CAP". It is not licensed at this time for "severe CAP", nor is an intravenous preparation available in the UK; hence, we have not assessed studies which have used intravenous moxifloxacin.

There are reported microbiological, pharmacokinetic and pharmacodynamic advantages for moxifloxacin compared with levofloxacin. ${ }^{357} 358$ [II] Clinical studies have generally shown equivalence with other oral antibiotics used for CAP. ${ }^{359-361 ~[\mathrm{Ib}]}$ [lb] [lb] One showed similar outcomes but fewer side effects when compared with oral amoxycillin (1 g tds) and/or clarithromycin. ${ }^{362}{ }^{[\mathrm{Ib}]}$ However, mainly in view of the increased risk of adverse hepatic reactions associated with moxifloxacin, the European Medicines Agency recommended in July 2008 that moxifloxacin should only be given in CAP when treatment with other antibiotics cannot be used. Similar advice is given in the British National Formulary (http://www.bnf.org/bnf/). ${ }^{363}$ 
Figure 9 Resistance (\%) to penicillin (high and intermediate), erythromycin and tetracycline among selected Streptococcus pneumoniae (blood and cerebrospinal fluid) isolates from laboratories reporting to the Public Health Laboratory Service.

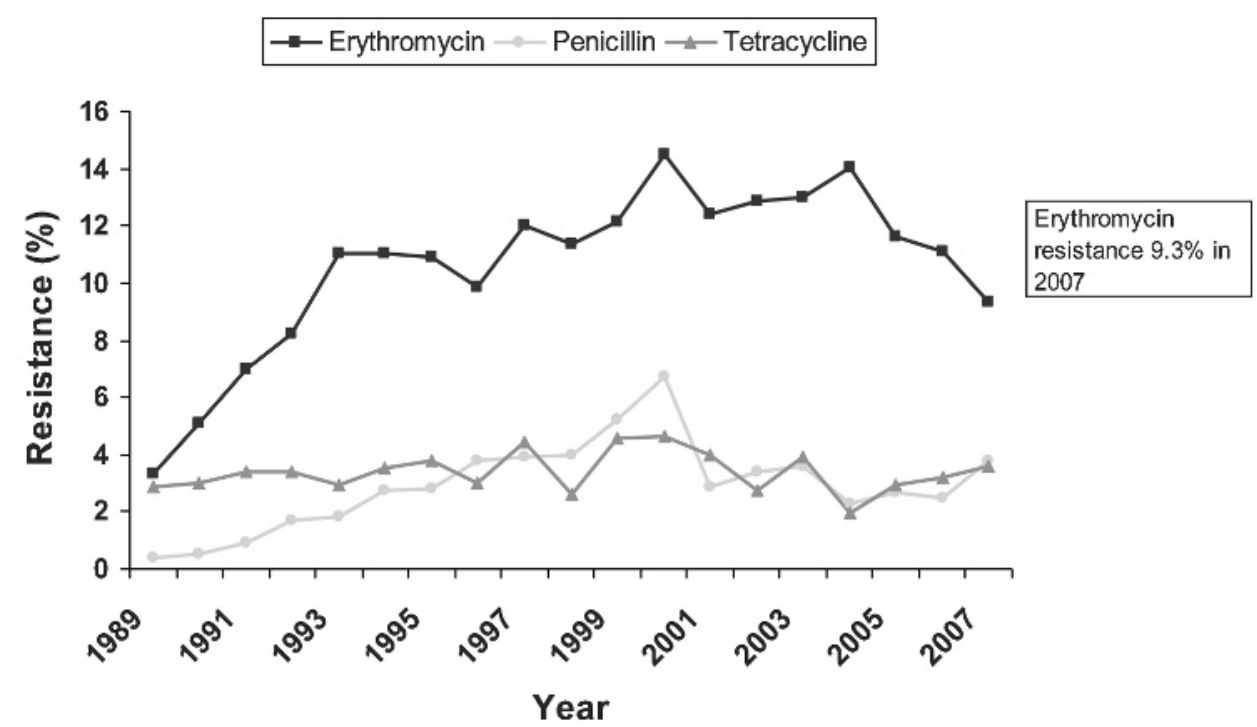

\subsection{Clinical studies of management and international differences in recommendations}

In defining the UK choice of empirical and specific therapy for CAP, it is apparent that the international differences in published recommendations cannot be entirely based on geographical variation in the distribution and antibiotic susceptibility of pathogens responsible for CAP. There is clearly variation in medical practice with regard to licensing, availability, choice, dose, route of administration and duration of therapy, which is more a reflection of local custom and practice than robust scientific evidence. The literature review for the period 1981-2008 provided only 19 acceptable articles relevant to the antibiotic management of CAP. ${ }^{277} 364-381$ [Ib] [Ib] [[b] [Ib] [III] [Ib]

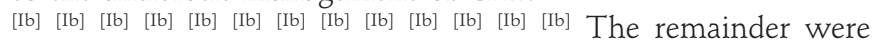
rejected for the following reasons: inadequately powered studies or a retrospective design, ${ }^{102}{ }^{382-400}$ non-blinded/non-randomised studies, ${ }^{274} 401-405$ antibiotic not available in the UK or withdrawn, ${ }^{357} 361$ 406-441 study population or management unrepresentative of normal clinical practice in the UK, ${ }^{37742-450}$ or they included mixed lower respiratory tract infections including CAP. ${ }^{377} 419451452$

Among the studies reviewed, few were conducted within a healthcare system comparable to that of the UK. Others were designed to support the licensing of new therapies. For this reason, they are primarily designed to demonstrate equivalence between the new agent and comparator therapy which may or may not have been selected in accordance with current standard management. This invariably makes it difficult to offer evidence-based recommendations since superiority of a particular regimen is rarely identified. Likewise, matters of differential safety for the various regimens is difficult to assess since this information is essentially a by-product of these licensing studies, is rarely standardised and has often not been compared with current standard therapy.

\subsection{Formulations of these recommendations}

The recommendations for treatment have been made on the basis of assessing a matrix of laboratory, clinical, pharmacokinetic and safety data, interpreted in an informed manner. While this remains an unsatisfactory basis for making robust evidencebased recommendations, it highlights the need for appropriate, prospective, randomised controlled studies designed to address the many key questions that will enable the management of CAP to be placed on a sounder basis. The responsibility for this presents a challenge to medical practitioners, healthcare systems, grant-giving bodies and industry. We have also only considered antibiotics licensed and available in the UK at the time we prepared these guidelines.

Currently, within the UK, control of hospital-acquired infection by $C$ difficile, MRSA and pathogens with extended $\beta$-lactamase activity is a priority of local and strategic health authorities. In line with the principles of prudent use of antibiotics, the current guidelines have been modified to discourage unnecessary use of broad-spectrum antibiotics, especially cephalosporins and fluoroquinolones. The development of refined techniques for severity stratification (see Section 6) have enabled a strategy of targeted antibiotic escalation, which should restrict the use of empirical potent broad-spectrum therapy to those cases in which it is necessary.

As stated elsewhere, "it is important to recognise that these are simply guidelines and reflect our interpretation of good practice within an evolving area. Guidelines cannot capture every clinical situation and it therefore remains the responsibility of the physician to balance the history and clinical features, assess the importance of risk factors and interpret local epidemiology and laboratory data in order to make the best judgement for an individual patient". 453 [IVa]

\section{(A) EMPIRICAL THERAPY}

\subsection{Empirical antibiotic choice for CAP treated in the community} Most patients with pneumonia are treated successfully in the community in the absence of any microbial definition of an infecting micro-organism(s). The decision to manage a patient in the community is based on a range of factors which include an assessment that the pneumonia is of low severity, that oral therapy is appropriate and will be complied with, and that the social circumstances and available care for an individual are satisfactory.

Empirical therapy is primarily directed at $S$ pneumoniae which remains the leading cause of CAP (see Section 3). ${ }^{243}$ [Ia] Apart from $M$ pneumoniae, atypical pathogens, Legionella spp and $\beta$ lactamase producing bacteria are uncommon in the community setting. $M$ pneumoniae exhibits epidemic periodicity every 
4-5 years largely, has a low mortality rate and affects younger persons. A policy for initial empirical therapy that aimed to always cover this pathogen was considered inappropriate.

For these reasons, as well as the issues of current practice, cost, wide experience and drug tolerance, amoxicillin remains the preferred agent. The alternative agents for those intolerant of amoxicillin are doxycycline and the macrolides clarithromycin and erythromycin. ${ }^{277}[\mathrm{Ib}]$ Recommendation of a tetracycline as an alternative therapy has been adopted on the basis of lower resistance rates among pneumococci (fig 9) and activity against atypical pathogens. Reduced gastrointestinal intolerance and an easier dosing schedule are the major reasons for recommending clarithromycin over erythromycin. Concern over the rising frequency of in vitro resistance (fig 9) of $S$ pneumoniae to macrolides (which is often linked to penicillin resistance) is recognised, yet published clinical evidence for clinical failure of macrolides in the treatment of pneumococcal pneumonia remains limited $^{454}$ [III] 455 and controversial. ${ }^{456} 457$ [IVa] [IVa]

The macrolides clarithromycin and azithromycin ${ }^{458}[\mathrm{IVa}]$ and the fluoroquinolones ${ }^{459}$ [IVa] have microbiological strengths in vitro, yet in published studies to date have not been shown to be more efficacious than standard therapy in treating patients with low severity CAP. Several meta-analyses (including a Cochrane review) of trials that have compared empirical antibiotic regimens with atypical pathogen coverage with regimens without atypical pathogen coverage in patients with low severity pneumonia have not found any benefit of regimens with atypical pathogen coverage in terms of survival or clinical efficacy. ${ }^{460-462}$

The association of $H$ influenzae and, to a much lesser extent, $M$ catarrhalis with acute exacerbations of COPD is recognised. ${ }^{463}$ ${ }^{[I I]}$ However, both remain uncommon causes of CAP. When CAP does arise with these pathogens, an even smaller percentage of such patients will be infected with $\beta$-lactamase producing strains. To illustrate the clinical significance of such resistance for managing CAP, it could be estimated that $5 \%$ of CAP cases may be caused by $H$ influenzae, of which $15 \%$ may be $\beta$ lactamase producing strains in the UK. Therefore, of 500 patients with CAP, only 4 may be infected with such antibioticresistant strains.

A view that specific pathogens are associated with other comorbid diseases (eg, $H$ influenzae and COPD) to increase the risk of CAP is not supported by the literature. For these reasons, these guidelines do not offer alternative regimens for patients with or without comorbid illness, while recognising that such diseases can affect the severity of CAP in an individual.

The current concern over the increasing prevalence of pneumococci with reduced susceptibility to penicillin is recognised. However, the incidence of highly resistant strains $(\mathrm{MIC} \geqslant 4 \mathrm{mg} / \mathrm{l})$ remains uncommon in the UK. Furthermore, the rarity of documented clinical failures among penicillinresistant pneumococcal pneumonia, if treated with adequate doses of penicillin, is the basis for endorsing oral amoxicillin as first-line therapy at a dosage of $500 \mathrm{mg}$ three times daily.

\section{Recommendations}

- For patients treated in the community, amoxicillin remains the preferred agent at a dose of $500 \mathrm{mg}$ three times daily. $[\mathrm{A}+]$

- Either doxycycline [D] or clarithromycin [A-] are appropriate as an alternative choice, and for those patients who are hypersensitive to penicillins.
- Those with features of moderate or high severity infection should be admitted urgently to hospital. [C]

\subsection{Should general practitioners administer antibiotics prior to hospital transfer in those patients who need admission?}

There is no direct evidence upon which to provide clear guidance on this question. There is, however, some circumstantial evidence to suggest that early antibiotics are of benefit in high severity pneumonia.

Delay in prescribing antibiotics for patients in hospital with diagnosed pneumonia is associated with a worse outcome $e^{464} 465$ [III] [III] and, in patients dying from CAP, the majority had not received prior antibiotics even though most had visited a general practitioner in the previous few days. In a national confidential enquiry into CAP deaths in young adults in England and Wales, 20 of the 27 fatal cases investigated had seen their general practitioner for the illness and only 9 had received antibiotics. ${ }^{46}$ [II] In the multicentre BTS study of CAP in 1982, none of the patients who died from pneumococcal pneumonia had received an antibiotic before admission. The authors concluded that some deaths may have been preventable and recommended that an antibiotic active against $S$ pneumoniae should be started as soon as pneumonia is recognised. ${ }^{6}{ }^{[\mathrm{bb}]}$ In a study from New Zealand, significantly fewer $(p=0.05)$ of those who died had received antibiotics before admission (20\%) compared with those who survived (42\%). ${ }^{467}{ }^{[\mathrm{Ib}]}$ Currently, less than half of adults admitted to hospital in the UK with high severity CAP have already received antibiotics from their general practitioner. ${ }^{42} 103$ [III] [III] Many deaths and requirements for assisted ventilation occur in the first few days of admission for high severity CAP. ${ }^{6}{ }^{37}{ }^{241}[\mathrm{Ib}][\mathrm{Ib}][\mathrm{Ib}]$ All of these studies provide further support to the suggestion that, in cases of diagnosed pneumonia, antibiotics should be given as early as possible, if necessary before hospital admission.

Delays do occur between general practitioner assessment in the community, arranging admission, confirmation of the diagnosis in hospital and the start of treatment. Probably these are inevitable and will be exacerbated by transport distances and ambulance availability and prioritisation, bed availability and triage in the medical assessment unit or emergency department. Delays between admission and receiving antibiotics of $>6 \mathrm{~h}$ have been reported for younger adults dying in hospital of CAP (average delay $260 \mathrm{~min}$ ), ${ }^{284}$ [III] although this study was conducted before medical assessment units were introduced into most UK hospitals.

From time to time, general practitioners do see patients who are severely ill with what appears to be pneumonia. In such circumstances, treatment should commence as soon as possible, providing it does not delay transfer to hospital. When general practitioners feel treatment in such circumstances is needed, it should aim to cover pneumococcal pneumonia-the commonest cause of high severity CAP — with intravenous penicillin G $1.2 \mathrm{~g}$ or oral amoxicillin $1 \mathrm{~g}$ orally (or clarithromycin $500 \mathrm{mg}$ in patients with penicillin sensitivity). General practitioners are likely to carry such antibiotics with them as parenteral penicillin is recommended as the immediate treatment for suspected meningococcal infection. Ambulance services should allocate to patients with pneumonia a high priority for transfer to hospital.

Prescribing antibiotics does have an influence on some microbiological investigations. ${ }^{6}[\mathrm{lb}]$ However, when general practitioners feel a patient is severely ill or circumstances suggest that delays in transfer will slow assessment and treatment in 
hospital, concern over the potential effect on subsequent investigations is not a reason to withhold treatment.

Inappropriate antibiotic use is a major concern both in community and hospital settings. The clinical likelihood of CAP therefore needs to be taken into account when considering antibiotic treatment at the time of hospital referral. It is important to state that, as with the whole of this document, these guidelines refer to patients with CAP and not to the much larger group of patients with non-pneumonic lower respiratory tract infection or exacerbations of COPD.

\section{Summary}

- Delays occur in the process of admitting patients to hospital with CAP and them receiving antibiotics. [III]

- There is direct and indirect evidence that administering antibiotics early is important in the outcome of CAP, particularly when the patient is assessed as being severely ill. [Ib]

- Less than half of patients admitted with high severity CAP have received antibiotics before admission, even though they may have seen their general practitioner. [III]

- Most deaths from CAP occur shortly after admission. [Ib]

- Pre-admission antibiotics can negatively influence the results of subsequent microbiological investigations, [Ib] but this is not seen as a reason for withholding antibiotics if the general practitioner feels they are indicated.

\section{Recommendations}

- For those patients referred to hospital with suspected CAP and where the illness is considered to be lifethreatening, general practitioners should administer antibiotics in the community. [D] Penicillin G $1.2 \mathrm{~g}$ intravenously or amoxicillin $1 \mathrm{~g}$ orally are the preferred agents.

- For those patients referred to hospital with suspected high severity CAP and where there are likely to be delays of over $6 \mathrm{~h}$ in the patient being admitted and treated in hospital, general practitioners should consider administering antibiotics in the community. [D]

\subsection{When should the first dose of antibiotic be given to patients admitted to hospital with CAP?}

The prompt administration of antibiotics to patients with proven CAP is good practice, matches patient expectation and is supported by some data demonstrating improved clinical outcomes. One large retrospective study of patients aged 65 years and older admitted with CAP demonstrated a 15\% reduction in 30-day mortality for patients treated within 8 h. ${ }^{464}$ ${ }^{[\mathrm{III}]}$ A further retrospective study in patients aged 65 years and older admitted with CAP and who had not received antibiotics before admission reported similar results. ${ }^{468}$ [III]

However, the diagnosis of CAP is not always evident on admission to hospital. One study reported that $22 \%$ of patients with CAP had atypical presentations which led initially to diagnostic uncertainty. ${ }^{469}$ Concerns have therefore been raised that undue emphasis on early antibiotic administration will lead to an increase in inaccurate diagnoses and consequently an increase in inappropriate antibiotic use. Studies from the USA have shown that, following a recommendation that the time to first antibiotic dose should be less than $4 \mathrm{~h}$ for all patients with CAP, there was a $39 \%$ reduction in the accuracy of emergency department diagnosis of CAP, an increase (from 20.6\% to
$28.3 \%$ ) in the number of patients diagnosed with "CAP" who actually had a normal chest radiograph ${ }^{470}$ and an increase in inappropriate antibiotic use. ${ }^{471}[\mathrm{III}]$

We have sought to offer recommendations that encourage prompt and appropriate antibiotic treatment of patients with CAP but that avoid forcing clinicians to diagnose and treat pneumonia when there is genuine uncertainty. The pivotal role of the chest radiograph in confirming or refuting a diagnosis of CAP in patients admitted with suspected CAP is emphasised in Section 5.2. It is also recognised that, in a minority of patients, atypical presentations of CAP may result in a delay in diagnosis.

\section{Recommendations}

- A diagnosis of CAP should be confirmed by chest radiography before the commencement of antibiotics in the majority of patients. Selected patients with lifethreatening disease should be treated based on a presumptive clinical diagnosis of CAP. In such instances, an immediate chest radiograph to confirm the diagnosis or to indicate an alternative diagnosis is indicated. [D]

- All patients should receive antibiotics as soon as the diagnosis of CAP is confirmed. D] This should be before they leave the initial assessment area (emergency department or medical assessment unit). The objective for any service should be to confirm a diagnosis of pneumonia with chest radiography and initiate antibiotic therapy for the majority of patients with CAP within $4 \mathrm{~h}$ of presentation to hospital. [B-]

\subsection{Empirical antibiotic choice for adults hospitalised with low severity CAP}

Approximately $20 \%$ of patients with CAP are hospitalised in the UK (see Section 2). The reasons for hospitalisation vary and include severity of the infection, an unsatisfactory response to treatment initiated by the general practitioner, significant comorbid illness and non-clinical reasons such as inappropriate home circumstances suitable for community management.

It is recognised that a significant number of patients with low severity pneumonia who might otherwise be adequately managed in the community are admitted to hospital for nonclinical reasons (advanced age, personal or family preference, inadequate home care or adverse social circumstances). Others will be admitted who have not received antibiotic therapy. They cannot be considered to have failed community treatment and, as such, initial therapy with a single agent is considered appropriate (see table 5). Furthermore, $M$ pneumoniae is an important contributor to the overall incidence of atypical pathogens but is an infrequent cause of CAP in elderly patients. This provides a further justification for simple monotherapy in the hospitalised elderly patient with low severity CAP. In all such circumstances, patient management requires careful clinical judgement and regular reviews.

Following initial assessment and empirical therapy, progress should be monitored carefully. The route and choice of antibiotic treatment will require adjustment, either by stepping up and broadening the spectrum of microbiological activity in the light of clinical deterioration or as a result of positive microbiological information, or stepping down with improvement as discussed below. The review of antibiotic therapy forms an obvious and essential part of the regular clinical review of patients with CAP. 


\section{Recommendations}

- Most patients with low severity CAP can be adequately treated with oral antibiotics. [C]

- Oral therapy with amoxicillin is preferred for patients with low severity CAP who require hospital admission for other reasons such as unstable comorbid illnesses or social needs. [D]

- When oral therapy is contraindicated, recommended parenteral choices include intravenous amoxicillin or benzylpenicillin, or clarithromycin. [D]

\subsection{Empirical antibiotic choice for adults hospitalised with moderate severity CAP}

The principles of antibiotic selection for moderate severity CAP managed in hospital are similar to those for its management in the community. The current guidelines incorporate a stratified approach based on disease severity (see Section 6). The predominant pathogen will be $S$ pneumoniae. However, overall atypical pathogens including Legionella spp account for approximately $20 \%$ of defined infections. Therefore, in patients who are admitted with moderate severity disease, oral therapy with a combined $\beta$-lactam/macrolide regimen is recommended.

When oral therapy is inappropriate, parenteral amoxicillin or penicillin $G$ are offered as alternatives to oral amoxicillin, with clarithromycin given twice a day as the preferred macrolide for parenteral therapy.

For patients intolerant of penicillin and in whom oral therapy is inappropriate, intravenous levofloxacin once daily or a combination of intravenous cephalosporin with intravenous clarithromycin are appropriate alternative choices. Institutions wishing to avoid the use of quinolones and cephalosporins in these patients may wish to consider monotherapy with intravenous clarithromycin, but must weigh the ecological benefit of this strategy against the risk of undertreating pneumococcal pneumonia (erythromycin resistance of about $9 \%$; see Section 8.3 and fig 9).

\section{Recommendations}

- Most patients with moderate severity CAP can be adequately treated with oral antibiotics. [C]

- Oral therapy with amoxicillin and a macrolide is preferred for patients with moderate severity CAP who require hospital admission. [D]

- Monotherapy with a macrolide may be suitable for patients who have failed to respond to an adequate course of amoxicillin prior to admission. Deciding on the adequacy of prior therapy is difficult and is a matter of individual clinical judgement. It is therefore recommended that combination antibiotic therapy is the preferred choice in this situation and that the decision to adopt monotherapy is reviewed on the "post take" round within the first $24 \mathrm{~h}$ of admission. [D]

-When oral therapy is contraindicated, the preferred parenteral choices include intravenous amoxicillin or benzylpenicillin, together with clarithromycin. [D]

- For those intolerant of penicillins or macrolides, oral doxycyline is the main alternative agent. Oral levofloxacin and oral moxifloxacin are other alternative choices. [D]

- When oral therapy is contraindicated in those intolerant of penicillins, recommended parenteral choices include levofloxacin monotherapy or a second-generation (eg, cefuroxime) or third-generation (eg, cefotaxime or ceftriaxone) cephalosporin together with clarithromycin. [D]

Table 5 Initial empirical treatment regimens for community acquired pneumonia (CAP) in adults

\begin{tabular}{llll}
\hline $\begin{array}{l}\text { Pneumonia severity (based on clinical } \\
\text { judgement supported by CURB65 } \\
\text { severity score) }\end{array}$ & Treatment site & Preferred treatment & Alternative treatment \\
\hline Low severity & Home & Amoxicillin $500 \mathrm{mg}$ tds orally & Doxycycline $200 \mathrm{mg}$ loading dose then $100 \mathrm{mg}$ \\
(eg, CURB65 $=0-1$ or CRB65 score & & & orally or clarithromycin $500 \mathrm{mg}$ bd orally
\end{tabular}

$=0,<3 \%$ mortality)

Low severity
(eg, CURB65 $=0-1,<3 \%$ mortality)

but admission indicated for reasons

other than pneumonia severity (eg,

social reasons/unstable comorbid

illness)

Moderate severity

(eg, CURB65 = 2, 9\% mortality)

High severity

(eg, CURB65 $=3-5,15-40 \%$

mortality)
Hospital

Amoxicillin $500 \mathrm{mg}$ tds orally

If oral administration not possible: amoxicillin $500 \mathrm{mg}$ tds IV
Doxycycline $200 \mathrm{mg}$ loading dose then $100 \mathrm{mg}$ od orally or clarithromycin $500 \mathrm{mg}$ bd orally

\footnotetext{
bd, twice daily; IV, intravenous; od, once daily; qds, four times daily; tds, three times daily.

* Following reports of an increased risk of adverse hepatic reactions associated with oral moxifloxacin, in October 2008 the European Medicines Agency (EMEA) recommended that moxifloxacin "should be used only when it is considered inappropriate to use antibacterial agents that are commonly recommended for the initial treatment of this infection". †Caution - risk of QT prolongation with macrolide-quinolone combination.
} 


\subsection{Empirical antibiotic choice for adults hospitalised with high severity CAP}

Mortality is greatly increased in those with high severity pneumonia (see Section 6). The illness may progress before microbiological information is available.

Preferred and alternative initial treatment regimens are summarised in table 5 and mostly include combination therapy with broad-spectrum $\beta$-lactams and a macrolide. While $S$ pneumoniae remains the predominant pathogen, $S$ aureus and Gram-negative enteric bacilli-although uncommon-carry a high mortality, ${ }^{243}$ [Ia] hence the recommendation for broadspectrum $\beta$-lactam regimens in those with high severity CAP. Patients hospitalised with CAP caused by Legionella spp are more likely to have high severity pneumonia. ${ }^{219}$ [Ia] For these reasons, the initial empirical antibiotic regimen should also always capture this pathogen within its spectrum of activity. ${ }^{472}$ Specific antibiotic recommendations for treating confirmed legionella infection are provided in Section 8.20. The near universal availability of $L$ pneumophila urine antigen testing means that a rapid diagnosis of $L$ pneumophila serogroup 1 infection can often be made early in the course of the admission. However, the urine antigen test may be negative on admission and is also insufficiently sensitive to exclude Legionnaires' disease, so empirical therapy for Legionnaires' disease should not be discontinued based solely on a negative antigen test.

Parenteral administration of antibiotic is recommended in those with high severity CAP regardless of the patient's ability or otherwise to take oral medication. This is to ensure prompt high blood and lung concentrations of antibiotic.

The preferred regimen includes co-amoxiclav. This agent has excellent activity against the pneumococcus, $H$ influenzae and $S$ aureus, as well as activity against anaerobes. In the current guidelines, cephalosporins and fluoroquinolones are included as alternative but not preferred choices, largely on the basis that their use has been consistently associated with hospital acquired infections, notably $C$ difficile associated disease. While coamoxiclav has also been shown to be associated with $C$ difficile infection, the Committee considers co-amoxiclav to be less likely to encourage $C$ difficile overgrowth compared with cephalosporins and fluoroquinolones. Furthermore, use of coamoxiclav enables transition from intravenous to oral therapy without switching class of agent, a property which might help physicians switch early to oral therapy which will also help limit $C$ difficile infection.

Efficacy data from prospective controlled clinical trials is not available. However, a retrospective study suggests a reduction in mortality for those treated with a third-generation cephalosporin plus a macrolide, ${ }^{473[\mathrm{III}]}$ although no additional benefit has been noted in another study. ${ }^{474}[\mathrm{II}]$

Levofloxacin is the only licensed and available intravenous fluoroquinolone in the UK at the time of writing. It is marketed in parenteral and oral formulations; since the latter is $98 \%$ bioavailable, this indicates that it can be used in high severity pneumonia provided there are no contraindications to oral administration. While it has modest activity against pneumococci in vitro, the published evidence for efficacy in high severity CAP is reassuring. ${ }^{198} 244267329330[\mathrm{lb}][\mathrm{lb}][\mathrm{lb}][\mathrm{lb}][\mathrm{lb}]$ However, until more clinical experience is available, we recommend combining it with another agent active against $S$ pneumoniae such as parenteral benzylpenicillin when managing high severity CAP.

A small minority of patients will be allergic to both penicillins and cephalosporins. Antibiotic selection is more difficult in these patients and should be discussed with the local respiratory and microbiology services.

\section{Recommendations}

- Patients with high severity pneumonia should be treated immediately after diagnosis with parenteral antibiotics. [B-]

- An intravenous combination of a broad-spectrum $\beta$ lactamase stable antibiotic such as co-amoxiclav together with a macrolide such as clarithromycin is preferred. [C]

- In patients allergic to penicillin, a second-generation (eg, cefuroxime) or third-generation (eg, cefotaxime or ceftriaxone) cephalosporin can be used instead of coamoxiclav, together with clarithromycin. [C]

8.13 When should the intravenous or the oral route be chosen?

Parenteral administration of antibiotics is widely and often unnecessarily used in managing hospitalised patients including those with CAP. ${ }^{369} 475$ [Ib] [IVa] Approximately $30-50 \%$ of patients admitted to hospital will initially require treatment with parenteral antibiotics. ${ }^{369}{ }^{[\mathrm{Ib}]}$ Apart from the discomfort to the patient of inserting intravenous devices, there are significant complications, notably infection. In addition, the total cost of parenteral regimens greatly exceeds orally administered therapy.

Factors determining the route of administration are summarised in box 3. Parenteral antibiotics are clearly indicated for patients unable to swallow, where there is concern about adequate absorption of drug from the gut and in the presence of severe pneumonia. However, many antibiotics are well absorbed following oral administration and achieve their maximum plasma concentration within 1-2 h.

\section{Recommendations}

- The oral route is recommended in those with low and moderate severity CAP admitted to hospital provided there are no contraindications to oral therapy. $[\mathrm{B}+]$

\subsection{When should the intravenous route be changed to oral?}

As stated above, parenteral antibiotic therapy is widely and often unnecessarily used among hospitalised patients with low and moderate severity pneumonia. This in part reflects custom and practice but, in addition, may be driven by too liberal an interpretation of the "criteria" for identifying high severity CAP for which parenteral agents are recommended. The current practice of medicine in emergency medical admissions may also be a factor in the choice of parenteral administration where it provides greater confidence to admitting junior medical staff that the patient is receiving the "best" management. Oral therapy was clearly more widely adopted in the past. ${ }^{475}$ However, published evidence indicating comparable efficacy of parenteral and oral regimens is limited, but has been shown for intravenous cefuroxime and oral levofloxacin. ${ }^{371}[\mathrm{Ib}]$

The choice and timing of any change to oral therapy will be affected by several factors. These include the absence of any contraindications to oral administration, the availability of any microbiological information regarding aetiology of the infection and clear evidence that the patient is responding to initial therapy.

There can be no rigid recommendation concerning the timing of transfer to oral therapy and further studies of this area are needed. ${ }^{438} 476477$ [II] [Ia] [II] Any decision must be individualised on the basis of assessing all factors. Nonetheless, the recommended guideline is that oral therapy be considered in a patient who has shown clear evidence of improvement and whose temperature has resolved for a period of $24 \mathrm{~h}$. The features indicating 
response to parenteral therapy are summarised in box 4. This policy will allow a significant proportion of patients with pneumonia to be safely transferred to an oral regimen after a period of initial parenteral therapy. ${ }^{279} 372393438476478$ [II] [Ib] [Ia] [II] [II] [IVa]

\section{Recommendations}

- Patients treated initially with parenteral antibiotics should be transferred to an oral regimen as soon as clinical improvement occurs and the temperature has been normal for $24 \mathrm{~h}$, providing there is no contraindication to the oral route. Pointers to clinical improvement are given in box 4 . $[\mathrm{B}+]$

- The choice of route of administration should be reviewed initially on the "post take" round and then daily. [D]

- Ward pharmacists could play an important role in facilitating this review by highlighting prescription charts where parenteral antibiotic therapy continues. [D]

\subsection{Which oral antibiotics are recommended on completion of intravenous therapy?}

The selection of agents for oral administration following initial intravenous therapy is based on antimicrobial spectrum, efficacy, safety and cost considerations. Although it may appear logical to select the oral formulation of a parenteral agent, this is not essential and such oral agents may not meet the criteria for selection. For macrolides, oral clarithromycin is better tolerated than oral erythromycin. ${ }^{364365[\mathrm{Ib}][\mathrm{Ib}]}$ A clinical judgement can be made whether to change to oral monotherapy in those who have responded favourably to parenteral combination therapy or where there is microbiological documentation of the nature of the infection, in which case the recommendations in table 6 should be adopted.

\section{Recommendations}

- The antibiotic choices for the switch from intravenous to oral are straightforward where there are effective and equivalent oral and parenteral formulations. [C]

- In the case of parenteral cephalosporins, the oral switch to co-amoxiclav $625 \mathrm{mg}$ three times daily is recommended rather than to oral cephalosporins. [D]

- For those treated with benzylpenicillin + levofloxacin, oral levofloxacin with or without oral amoxicillin $500 \mathrm{mg}-1.0 \mathrm{~g}$ three times daily is recommended. [D]

\subsection{How long should antibiotics be given for?}

The precise duration of antibiotic therapy for the management of microbiologically documented and non-documented CAP is

\section{Box 3 Indications for parenteral and oral antibiotic therapy}

\section{of adult CAP}

Parenteral therapy

- High severity pneumonia

- Impaired consciousness

- Loss of swallowing reflex

- Functional or anatomical reasons for malabsorption

Oral therapy

- Community managed

- Hospital managed, non-severe with no other contraindications not supported by robust evidence. One RCT of adults with low to moderate severity pneumonia treated with amoxicillin reported similar efficacy with a 3-day intravenous course as with an 8-day (3 days intravenous followed by 5 days oral) course. ${ }^{479}$ The Summary of Product Characteristics (formerly the Drug Data Sheets) for many agents used in the treatment of CAP mention a range of treatment durations which sometimes differ internationally. Intracellular pathogens responsible for pneumonia sometimes respond slowly, and hence a 2-week treatment regimen has been proposed for atypical pathogens.

The aim of antibiotic therapy is to ensure elimination of the target pathogen in the shortest time. In uncomplicated infections this is likely to occur rapidly (within 3 days) with many common respiratory pathogens such as $S$ pneumoniae. The resolution of pneumonia involves not only the elimination of the invading pathogen and its products, but also the subsidence of the host inflammatory response which together are responsible for the many clinical and radiographic features of pneumonia.

Until we have more precise methods to reliably identify microbiological and clinical end-points, the duration of therapy will remain subject to clinical judgement. For these reasons, the duration of therapy will vary by individual patient, disease severity and speed of resolution.

\section{Recommendations}

- For community managed and for most patients admitted to hospital with low or moderate severity and uncomplicated pneumonia, 7 days of appropriate antibiotics is recommended. [C]

- For those with high severity microbiologically-undefined pneumonia, 7-10 days treatment is proposed. This may need to be extended to 14 or 21 days according to clinical judgement; for example, where $S$ aureus or Gram-negative enteric bacilli pneumonia is suspected or confirmed. [C]

\subsection{Failure of initial empirical therapy}

In those patients who fail to respond to initial empirical therapy, several possibilities need to be considered, the first of which is whether the correct diagnosis has been made. Clinical and radiographic review is recommended for patients managed in the community and in hospital to look for secondary diagnoses or complications of CAP such as pleural effusion/ empyema, lung abscess or worsening pneumonic shadowing. This aspect is considered in detail in Section 9.

The initial empirical antibiotic regimen may need to be reassessed. However, compliance with and adequate absorption of an oral regimen should first be considered.

Microbiological data should be reviewed and further specimens examined with a view to excluding less common pathogens such as $S$ aureus, atypical pathogens, Legionella spp, viruses and Mycobacteria spp. It should also be noted that mixed infections can arise in approximately $10 \%$ of patients hospitalised with CAP. In the absence of any microbiological indicators of infection, the management of those failing initial empirical therapy will vary according to the severity of illness at reassessment. In patients with low severity pneumonia managed in the community, a macrolide could be substituted for amoxicillin. However, when the patient's condition has deteriorated, admission to hospital should be considered.

In the patient with low or moderate severity pneumonia managed in hospital, the addition of a macrolide is recommended 


\section{Box 4 Features indicating response to initial empirical parenteral therapy permitting consideration of oral antibiotic substitution}

- Resolution of fever for $>24 \mathrm{~h}$

- Pulse rate $<100$ beats/min

- Resolution of tachypnoea

- Clinically hydrated and taking oral fluids

- Resolution of hypotension

- Absence of hypoxia

- Improving white cell count

- Non-bacteraemic infection

- No microbiological evidence of legionella, staphylococcal or Gram-negative enteric bacilli infection

- No concerns over gastrointestinal absorption

in those patients initially managed with amoxicillin alone. Changing to doxycyline or a new fluoroquinolone such as levofloxacin are alternatives.

In the patient with high severity pneumonia already receiving a $\beta$-lactam/clarithromycin regimen, the addition of levofloxacin should be considered. In addition, urgent referral to a respiratory physician should be made for clinical assessment including the possible need for bronchoscopic sampling.

\section{Recommendations}

- When a change in empirical antibiotic therapy is considered necessary, a macrolide could be substituted for or added to the treatment for those with low severity pneumonia treated with amoxicillin monotherapy in the community or in hospital. [D]

- For those with moderate severity pneumonia in hospital on combination therapy, changing to doxycycline or a fluoroquinolone with effective pneumococcal cover are alternative options. [D]

- Adding a fluoroquinolone is an option for those with high severity pneumonia not responding to a $\beta$-lactam/ macrolide combination antibiotic regimen. [D]

\subsection{Antibiotic stewardship and avoiding inappropriate antibiotic prescribing for CAP}

Inappropriate antibiotic prescribing is a driver for antibiotic resistance and increases the likelihood of development of $C$ difficile infection. Given the frequency of admissions to hospital for suspected CAP and the difficulty in identifying a causative pathogen often leading to initial empirical broad-spectrum antibiotic therapy, it is timely to offer guidance to limit inappropriate prescribing in the context of the management of patients with CAP.

Ensuring an accurate diagnosis of CAP is the most important issue in relation to the avoidance of inappropriate antibiotic prescribing. One study reported that $29 \%$ of hospitalised

Table 6 Recommended treatment of microbiologically documented pneumonia and aspiration pneumonia (local specialist advice should also be sought*)

\begin{tabular}{|c|c|c|}
\hline Pathogen & Preferred & Alternative \\
\hline$S$ pneumoniae & $\begin{array}{l}\text { Amoxicillin } 500 \mathrm{mg}-1.0 \mathrm{~g} \dagger \mathrm{tds} \text { orally or } \\
\text { benzylpenicillin } 1.2 \mathrm{~g} \text { qds IV }\end{array}$ & $\begin{array}{l}\text { Clarithromycin } 500 \mathrm{mg} \text { bd orally or cefuroxine } 0.75-1.5 \mathrm{~g} \\
\text { tds IV or cefotaxime } 1-2 \mathrm{~g} \text { tds IV or ceftriaxone } 2 \mathrm{~g} \text { od IV }\end{array}$ \\
\hline $\begin{array}{l}\text { M pneumoniae } \\
\text { C pneumoniae }\end{array}$ & Clarithromycin $500 \mathrm{mg}$ bd orally or IV & $\begin{array}{l}\text { Doxycycline } 200 \mathrm{mg} \text { loading dose then } 100 \mathrm{mg} \text { od orally } \\
\text { or fluoroquinolonef orally or IV }\end{array}$ \\
\hline $\begin{array}{l}\text { C psittaci } \\
\text { C burnetii }\end{array}$ & $\begin{array}{l}\text { Doxycycline } 200 \mathrm{mg} \text { loading dose then } 100 \mathrm{mg} \text { od } \\
\text { orally }\end{array}$ & Clarithromycin $500 \mathrm{mg}$ bd orally or $500 \mathrm{mg}$ bd IV \\
\hline Legionella spp & Fluoroquinolone orally or IV $\$ \S$ & $\begin{array}{l}\text { Clarithromycin } 500 \mathrm{mg} \text { bd orally or IV (or, if necessary, } \\
\text { azithromycin in countries where this antibiotic is used for } \\
\text { managing pneumonia) }\end{array}$ \\
\hline$H$ influenzae & $\begin{array}{l}\text { Non- } \beta \text {-lactamase-producing: amoxicillin } 500 \mathrm{mg} \text { tds } \\
\text { orally or IV } \\
\beta \text {-lactamase-producing: co-amoxiclav } 625 \mathrm{mg} \text { tds } \\
\text { orally or } 1.2 \mathrm{~g} \text { tds IV }\end{array}$ & $\begin{array}{l}\text { Cefuroxime } 750 \mathrm{mg}-1.5 \mathrm{~g} \text { tds IV or cefotaxime } 1-2 \mathrm{~g} \text { tds } \\
\text { IV or ceftriaxone } 2 \mathrm{~g} \text { od IV or fluoroquinolone: orally or IV }\end{array}$ \\
\hline $\begin{array}{l}\text { Gram-negative } \\
\text { enteric bacilli }\end{array}$ & $\begin{array}{l}\text { Cefuroxime } 1.5 \mathrm{~g} \text { tds or cefotaxime } 1-2 \mathrm{~g} \text { tds IV or } \\
\text { ceftriaxone } 1-2 \mathrm{~g} \text { bd IV }\end{array}$ & $\begin{array}{l}\text { Fluoroquinolonet IV or imipenem } 500 \mathrm{mg} \text { qds IV or } \\
\text { meropenem } 0.5-1.0 \mathrm{~g} \text { tds IV }\end{array}$ \\
\hline$P$ aeruginosa & $\begin{array}{l}\text { Ceftazidime } 2 \mathrm{~g} \text { tds IV plus gentamicin or tobramycin } \\
\text { (dose monitoring) }\end{array}$ & $\begin{array}{l}\text { Ciprofloxacin } 400 \mathrm{mg} \text { bd IV or piperacillin } 4 \mathrm{~g} \text { tds IV, plus } \\
\text { gentamicin or tobramycin (dose monitoring) }\end{array}$ \\
\hline$S$ aureus & $\begin{array}{l}\text { Non-MRSA: flucloxacillin } 1-2 \mathrm{~g} \text { qds IV } \pm \text { rifampicin } \\
600 \mathrm{mg} \text { od or bd orally/IV }\end{array}$ & $\begin{array}{l}\text { MRSA: vancomycin } 1 \mathrm{~g} \text { bd IV (dose monitoring) or } \\
\text { linezolid } 600 \mathrm{mg} \text { bd IV or teicoplanin } 400 \mathrm{mg} \text { bd IV } \pm \\
\text { rifampicin } 600 \mathrm{mg} \text { od } \text { or bd orally/IV }\end{array}$ \\
\hline $\begin{array}{l}\text { Aspiration } \\
\text { pneumonia }\end{array}$ & Co-amoxiclav $1.2 \mathrm{~g}$ tds IV & Seek local microbiology advice \\
\hline
\end{tabular}

bd, twice daily; IV, intravenous; od, once daily; tds, three times daily.

*Treatment can be modified once the results of sensitivity testing are available.

$\uparrow A$ higher dose of $1.0 \mathrm{~g}$ tds is recommended for infections documented to be caused by less susceptible strains (minimum inhibitory concentration $>1.0 \mathrm{mg} / \mathrm{l})$.

:Currently UK licensed and available suitable fluoroquinolones include ciprofloxacin, ofloxacin and levofloxacin. Moxifloxacin can be used for patients who cannot be treated or have failed treatment with other antibacterials.

$\S$ Specifically for legionella pneumonia, the large majority of published experience regarding the efficacy of fluoroquinolones is only with levofloxacin. For high severity or life-threatening legionella pneumonia, combination therapy including the preferred and an alternative antibiotic can be considered for the first few days (see text for further details). Rifampicin is not recommended on its own but could be considered as the second additional antibiotic. 
patients treated for CAP did not have radiographic abnormalities. $^{471}[\mathrm{III}]$

In patients with CAP, broad-spectrum intravenous antibiotics should only be used following careful consideration, taking into account severity of illness, likely pathogens involved and patient-specific factors such as the ability to tolerate oral medication. Whenever appropriate, narrow-spectrum oral antibiotics should be used preferentially.

\section{Recommendations}

- The diagnosis of CAP and the decision to start antibiotics should be reviewed by a senior clinician at the earliest opportunity. There should be no barrier to discontinuing antibiotics if they are not indicated. [D]

- The indication for antibiotics should be clearly documented in the medical notes. [D]

- The need for intravenous antibiotics should be reviewed daily. [D]

- De-escalation of therapy, including the switch from intravenous to oral antibiotics, should be considered as soon as is appropriate, taking into account response to treatment and changing illness severity. [D]

- Strong consideration should be given to narrowing the spectrum of antibiotic therapy when specific pathogens are identified or when the patient's condition improves. [D]

- Where appropriate, stop dates should be specified for antibiotic prescriptions. [D]

\section{(B) SPECIFIC PATHOGEN-DIRECTED ANTIBIOTIC THERAPY}

\subsection{What are the optimum antibiotic choices when specific pathogens have been identified?}

In routine clinical practice, only about one-third to one-quarter of patients with CAP admitted to hospital will be defined microbiologically. Of these, some such as mycoplasma, chlamydophila and $C$ burnetii infection will be diagnosed late in the illness on the basis of seroconversion, reducing the opportunity for early targeted therapy. Among patients managed in the community, very few will be microbiologically defined.

When a pathogen has been identified, specific therapy as summarised in table 6 is proposed. In transferring patients from empirical to pathogen-targeted therapy, the regimen and route of administration will be determined by the continued need for parenteral therapy and known drug intolerance. Hence, table 6 provides preferred and alternative regimens for intravenous or oral administration. However, it should be remembered that approximately $10 \%$ (see Section 3 ) of infections will be of mixed aetiology, although many such co-pathogens will be viral and hence not influenced by antibiotic choice. These recommendations are again based on a synthesis of information which includes in vitro activity of the drugs, appropriate pharmacokinetics and clinical evidence of efficacy gleaned from a variety of studies. The choice of agent may be modified following the availability of sensitivity testing or following consultation with a specialist in microbiology, infectious disease or respiratory medicine.

Currently, $S$ pneumoniae highly resistant to penicillin (MIC $\geqslant 4 \mathrm{mg} / \mathrm{l}$ ) is uncommon in the UK. $S$ aureus is an uncommon cause of CAP in the UK. Most community isolates are methicillin-sensitive, although the recent increase in MRSA in hospitalised patients may result in subsequent readmission with an MRSA infection which may include CAP. Options for methicillin-sensitive and methicillin-resistant infections are based on parenteral administration in view of the serious nature of staphylococcal pneumonia.

\section{Recommendations}

- If a specific pathogen has been identified, the antibiotic recommendations are summarised in table $6[\mathrm{C}]$.

\subsection{Specific issues regarding the management of Legionnaires' disease}

Legionella infection is unique as a cause of CAP owing to the mode of spread and the importance of trying to identify the source to prevent further cases. The infection is acquired by inhaling water mist containing legionella bacteria, which are commonly found in natural and manmade water systems. Common sources include drift from evaporative cooling towers and aerosols generated from showers, aerated hot tubs, humidifiers and potable water. In addition, the propensity to cause severe illness including multiple organ failure and the lack of response to $\beta$-lactam antibiotics suggests that specific guidance will be helpful to clinicians managing a case.

Detection of legionella urinary antigen using a rapid technique has revolutionised the early diagnosis of pneumonia caused by $L$ pneumophila serogroup 1 which is responsible for over $90 \%$ of community cases in the UK and Europe. This is a highly sensitive and specific test, particularly in patients who are unwell enough or who have been ill long enough to require hospital admission (see Section 6). Although urinary antigen detection is now the most valuable and frequently used diagnostic test, sputum culture still has a vital role in diagnosing infection caused by other legionella serogroups and species. It is also invaluable for matching legionella species and strains identified from cases, with those positive water cultures from a potential source. This can help prevent further cases of infection. It is therefore important to send off sputum or respiratory secretions specifically for legionella culture, even in proven cases and after appropriate antibiotics have started (see Section 5.11.5)

Legionnaires' disease is not currently a notifiable disease in the UK, but urgent action to investigate the source of infection is essential. In proven cases of legionella pneumonia, the clinician should liaise with the clinical microbiologist to confirm that the local Health Protection Unit has been informed. The Health Protection Unit should initiate immediate investigations and this will include recording details of recent travel and activities within the 2 weeks before illness onset (the usual incubation period is $2-10$ days) from the patient or their relatives to assist epidemiological investigation. Clinicians should help by explaining the need for this to patients and relatives; this is important, especially at a time when the patient may be very unwell and their relatives distressed. The Health Protection Agency (HPA) will also request a clinical information sheet to be completed which provides very valuable (anonymous) clinical and outcome data to update the ongoing national and European data sets which are available from the HPA website (www.hpa.org.uk) or, for Scotland, from Health Protection Scotland (www.hps.scot.nhs.uk) and the European Working Group for Legionella Infections (www.ewgli.org). Further details about the investigation of a potential source of 
legionella infection and the management of sporadic cases can be found at the HPA website (www.hpa.org.uk) under the section Infectious Diseases $\rightarrow$ Infections $A-Z \rightarrow$ Legionnaires' disease $\rightarrow$ Guidelines.

There are no robust trials comparing the efficacy of different antibiotics for treating Legionnaires' disease, but in vitro studies and clinical experience in the form of observational case series support the use of quinolones, macrolides, azithromycin and rifampicin, with fluoroquinolones being the most effective agents. By contrast, antibiotics are not required for the nonpneumonic form of legionellosis-pontiac fever-which presents as a self-limiting flu-like illness with an incubation period of only 1-2 days.

A recent paper regarding the antibiotic therapy for Legionnaires' disease ${ }^{480}$ [IVa] reviewed the combined results from three recent observational studies of 458 patients with Legionnaires' disease managed with either a macrolide or a fluoroquinolone (mostly levofloxacin in an initial dose of $500 \mathrm{mg}$ twice daily ${ }^{481-483 ~[I I] ~[I I] ~[I I] ~}$ and concluded that fluoroquinolones appeared to have significant advantages over macrolides when comparing the end points of defervescence, side effects and hospital stay; however, the mortality rate was similar.

As the evidence base regarding the clinical efficacy of specific antibiotics is weak, consensus is used to make recommendations. Fluoroquinolones are regarded as the antibiotic of choice for proven legionella infection and, in such cases, their use should not be restricted by general ecological concerns over quinolone use.

For low and moderate severity community acquired legionella infection, an oral fluoroquinolone is recommended and should be prescribed unless there is definite patient intolerance which prevents its use. In this unusual circumstance, a macrolide is an alternative. (azithromycin is rarely used in the UK for CAP but is an alternative in countries where it is recommended for CAP).

For the management of high severity or life-threatening legionella pneumonia, a fluoroquinolone should be used wherever possible. There are no robust data on the use of combination antibiotics for high severity legionella pneumonia. However, in most circumstances, patients will already be on empirical combined antibiotic therapy for severe CAP which includes a macrolide (clarithromycin) at the time the diagnosis is made. Thus, with the knowledge of the significant mortality of high severity legionella pneumonia and the possibility of dual infection, continuing combined antibiotic therapy with a macrolide in addition to the fluoroquinolone is recommended during the crucial first few days. Azithromycin (in countries where this antibiotic is used for pneumonia) is an alternative to clarithromycin. However, clinicians should be alert to the potential small risk of cardiac electrophysiological abnormalities including prolongation of the QT interval on the ECG with the recommended combination, ${ }^{484}$ [IVa] particularly if other proarrhythmic risk factors are present. Rifampicin has traditionally also been offered as an alternative antibiotic to add as the second antibiotic in combined therapy and we include it as a recommended alternative. However, one small cohort observational study of 32 patients with legionella pneumonia reported that the addition of rifampicin to clarithromycin therapy offered no additional benefit in terms of recovery and that those receiving rifampicin had a longer length of stay in hospital. ${ }^{485}[\mathrm{III]}$ With parenteral rifampicin there is also a risk of hyperbilirubinaemia, which usually resolves on stopping the drug. ${ }^{486}$ [III] Further studies are indicated in the optimal treatment of high severity legionella pneumonia.
It has become accepted dogma that prolonged antibiotic therapy of 2-3 weeks is needed for legionella pneumonia to prevent relapses. This is based on a few anecdotal cases reported following the Philadelphia outbreak in 1976, largely involving immunocompromised patients. ${ }^{487}$ [III] However, there is no evidence to support the statement that patients with community acquired legionella pneumonia require longer therapy than other patients with CAP, and the duration of therapy should be guided as usual by clinical judgement.

\section{Recommendations}

- As soon as a diagnosis of legionella pneumonia has been made, the clinician should liaise with the clinical microbiologist to confirm that the local Health Protection Unit has been informed. The Health Protection Unit is responsible for promptly investigating the potential sources of infection. [D]

- The clinician should assist, where appropriate, in the gathering of clinical and epidemiological information from the patient and their relatives to aid the source investigation. [D]

- Sputum or respiratory secretions should be sent off specifically for legionella culture in proven cases, even after appropriate antibiotics have started. [D]

- For low and moderate severity community acquired legionella pneumonia, an oral fluoroquinolone is recommended. In the unusual case when this is not possible due to patient intolerance, a macrolide is an alternative. [D] Antibiotics are not required for the non-pneumonic self-limiting form of legionellosispontiac fever. [D]

- For the management of high severity or life threatening legionella pneumonia, a fluoroquinolone is recommended. For the first few days this can be combined with a macrolide (azithromycin is an option in countries where it is used for pneumonia) or rifampicin as an alternative. [D] Clinicians should be alert to the potential small risk of cardiac electrophysiological abnormalities with quinolone-macrolide combinations.

- Duration of therapy should be as for microbiologicallyundefined CAP (for those with low to moderate severity pneumonia, 7 days treatment is proposed; for those with high severity pneumonia, 7-10 days treatment is proposed-this may need to be extended to 14 or 21 days, see Section 8.16 ) and should be guided by clinical judgement. [D]

\subsection{Specific issues regarding Panton-Valentine Leukocidin- producing Staphylococcus aureus}

Necrotising pneumonia caused by a Panton-Valentine Leukocidin (PVL)-producing strain of $S$ aureus (PVL-SA, either MSSA or MRSA) is rare in the UK and Europe. If strongly suspected, blood cultures and respiratory samples should have an urgent Gram stain and be cultured on non-selective media to aid recovery of pathogens. Clinicians strongly suspecting infection due to PVL-SA should liaise with their local microbiology laboratory to ensure sensitivity testing and toxin gene profiling are carried out.

Detailed guidance on the specific management of patients with necrotising pneumonia due to PVL-SA is available from the HPA website (http://www.hpa.org.uk/web/HPAwebFile/ HPAweb_C/1218699411960). 


\section{Recommendations}

- PVL-SA infection is a rare cause of high severity pneumonia and can be associated with rapid lung cavitation and multiorgan failure. Such patients should be considered for critical care admission. [D]

- If PVL-SA necrotising pneumonia is strongly suspected or confirmed, clinicians should liaise urgently with the microbiology department in relation to further antibiotic management and consider referral to the respiratory medicine department for clinical management advice. [D]

- Current recommendations for the antibiotic management of strongly suspected necrotising pneumonia include the addition of a combination of intravenous linezolid $600 \mathrm{mg}$ bd, intravenous clindamycin $1.2 \mathrm{~g}$ qds and intravenous rifampicin $600 \mathrm{mg}$ bd to the initial empirical antibiotic regimen. As soon as PVL-SA infection is either confirmed or excluded, antibiotic therapy should be narrowed accordingly. [D]

\section{SECTION 9 COMPLICATIONS AND FAILURE TO IMPROVE}

9.1 What factors and action should be considered in patients who fail to improve in hospital?

For patients in hospital with CAP, the median time to improvement in heart rate and blood pressure is 2 days and in temperature, respiratory rate and oxygen saturation is 3 days. Failure to improve with initial management may occur in 6$24 \%$ of patients. ${ }^{488} 489$ [II] [II]

Independent risk factors for failure to improve that have been identified include multilobar involvement, cavitating pneumonia, presence of a pleural effusion, co-existing liver disease, cancer or neurological disease, aspiration pneumonia, legionella pneumonia, Gram-negative pneumonia, leucopenia, high disease severity on admission and inappropriate antimicrobial therapy. ${ }^{488-490}[\mathrm{II}][\mathrm{II}][\mathrm{II}]$

Patients who fail to improve have a poorer prognosis. Studies have reported a mean increase in length of hospital stay of 4 days $^{489}[\mathrm{II}]$ and an increase in mortality. ${ }^{490}[\mathrm{II}]$

Failure to improve should lead to consideration of various possibilities summarised in box 5 .

\section{Recommendations}

- For patients who fail to improve as expected, there should be a careful review by an experienced clinician of the clinical history, examination, prescription chart and results of all available investigation results. [D]

- Further investigations, including a repeat chest radiograph, C-reactive protein and white cell count and further specimens for microbiological testing should be considered in the light of any new information after the clinical review. [D]

- Referral to a respiratory physician should be considered. [D]

\subsection{What are the common complications of CAP?}

A brief description of the common complications of CAP is given below. Complications associated with specific infections are summarised in table 7 .

\subsubsection{Pleural effusion and empyema}

Parapneumonic effusions develop in $36-57 \%$ of bacterial pneumonias admitted to hospital and can be the cause of persisting pyrexia despite adequate antibiotic treatment. ${ }^{491}$ [II]
The presence of bilateral pleural effusions in CAP is associated with increased mortality. ${ }^{259}$ [II] Although most effusions will resolve with antibiotic therapy alone, it is recommended that thoracocentesis is performed promptly in patients with parapneumonic effusion admitted to hospital. Those patients shown to have an empyema (defined as the detection of cloudy fluid, pus or organisms on Gram stain or culture ${ }^{492}[\mathrm{III})$ or a complicated parapneumonic effusion (defined as clear pleural fluid with a $\mathrm{pH}<7.2^{493}{ }^{[\mathrm{II}]}$ ) should then have early and effective pleural space drainage. Pleural fluid for $\mathrm{pH}$ should be collected anaerobically in a heparinised blood gas syringe and measurement is performed in a blood gas analyser.

The incidence of empyema in patients admitted to hospital with CAP in a Canadian study was $0.7-1.3 \%$. The in-hospital mortality in these patients was $4.2 \% .{ }^{494}[\mathrm{II}]$ Other data indicate an increase in the incidence of patients hospitalised with empyema over the last decade. 495496 [III] [III]

Recognition of empyema is important as delayed thoracocentesis and chest tube drainage lead to longer and more costly hospitalisation. ${ }^{491}$ [III] Further details on the management of empyema are available in the BTS guidelines on the management of pleural infection. ${ }^{497}$

\section{Recommendations}

- Early thoracocentesis is indicated for all patients with a parapneumonic effusion. [D]

- Those found to have an empyema or clear pleural fluid with $\mathrm{pH}<7.2$ should have early and effective pleural fluid drainage. [C]

- The BTS guidelines for the management of pleural infection should be followed. [D]

\subsubsection{Lung abscess}

Lung abscess is a rare complication of CAP, being seen most commonly in the debilitated or alcoholic patient and following aspiration. Infection with anaerobic bacteria, $S$ aureus, Gramnegative enteric bacilli or $S$ milleri (in the presence of poor dental hygiene) should be considered. Most patients respond to appropriate antibiotics. A prolonged course of antibiotics may be required, although there is a lack of evidence on which to base firm recommendations regarding the optimum duration of antimicrobial therapy. Early surgical drainage via pneumonotomy may occasionally be needed.

\section{Recommendations}

- Less usual respiratory pathogens including anaerobes, $S$ aureus, Gram-negative enteric bacilli and $S$ milleri should be considered in the presence of lung abscess. [D]

- Prolonged antibiotic therapy of up to 6 weeks depending on clinical response and occasionally surgical drainage should be considered. [D]

\subsubsection{Metastatic infection}

Patients with septicaemia associated with pneumonia can occasionally develop metastatic infection. Meningitis, peritonitis, endocarditis and septic arthritis have all been reported. Purulent pericarditis can occur, usually in direct relation to an empyema.

Most such complications can be detected by careful history and examination. 


\section{Box 5 Reasons for failure to improve as expected}

Incorrect diagnosis or complicating condition

- Common

- Pulmonary embolism/infarction

- Pulmonary oedema

- Bronchial carcinoma

- Bronchiectasis

- Slow response in the elderly patient

- Uncommon

- Pulmonary eosinophilia/eosinophilic pneumonia

- Cryptogenic organising pneumonia

- Pulmonary alveolar haemorrhage

- Foreign body

- Congenital pulmonary abnormality leg, lobar sequestration)

Unexpected pathogen or pathogens not covered by antibiotic choice

- Pathogens always resistant to common antibiotics (eg, an "atypical pathogen" not responding to penicillin)

- Pathogens sometimes resistant to commonly used antibiotics (eg, ampicillin-resistant $H$ influenzae; penicillin-resistant $S$ pneumoniae; mycobacteria)

Antibiotic ineffective or causing allergic reaction

- Poor absorption of oral antibiotic

- Inadequate dose

- Antibiotic hypersensitivity

- Patient not receiving or taking prescribed antibiotic

Impaired local or systemic defences

- Local (eg, bronchiectasis, endobronchial obstruction, aspiration)

- Systemic immune deficiency (eg, HIV infection, hypogammaglobulinaemia, myeloma)

Local or distant complications of CAP

- Pulmonary

- Parapneumonic effusion

- Empyema

- Lung abscess

- Adult respiratory distress syndrome

- Extrapulmonary

- Phlebitis at intravenous cannula site

- Metastatic infection

- Septicaemia

- End organ sequelae of septicaemia (eg, renal failure)

\section{Overwhelming infection}

Improvement expected too soon

- For example, in elderly patients

\section{SECTION 10 PREVENTION AND VACCINATION}

\subsection{Influenza and pneumococcal vaccination}

The prevention of CAP, particularly in those considered at high risk of infection, is an important issue in the overall management of CAP. The Joint Committee on Vaccination and Immunisation is an independent expert advisory committee that advises the Secretaries of State of Health, Scotland, Wales and Northern Ireland on matters relating to communicable
Table 7 Some complications associated with specific infections

\begin{tabular}{ll}
\hline Pathogen & Complications \\
\hline S pneumoniae & $\begin{array}{l}\text { Septicaemia, pyopneumothorax, pericarditis/endocarditis, } \\
\text { meningitis/brain abscess, peritonitis, arthritis, herpes } \\
\text { labialis } \\
\text { Meningoencephalitis, aseptic meningitis, Guillain-Barré } \\
\text { syndrome, transverse myelitis, cerebellar ataxia, } \\
\text { ascending polyneuropathy, pericarditis, myocarditis, } \\
\text { diarrhoea, haemolytic anaemia, skin rashes, } \\
\text { polyarthropathy, hepatitis, pancreatitis, splenomegaly, } \\
\text { acute glomerulonephritis, haemorrhagic myringitis } \\
\text { Confusion, encephalomyelitis, Guillain-Barré syndrome, } \\
\text { cerebellar signs, pericarditis, hyponatraemia, renal failure, } \\
\text { rhabdomyolysis and myositis, diarrhoea, polyarthropathy } \\
\text { Jaundice/abnormal liver function, pancreatitis, } \\
\text { thrombocytopenia } \\
\text { Optic neuritis, hepatitis, haemolytic anaemia, } \\
\text { osteomyelitis, endocarditis with chronic infection } \\
\text { Pneumatoceles and/or pneumothorax (especially in } \\
\text { children), septicaemia, lung abscess, metastatic infection }\end{array}$ \\
&
\end{tabular}

disease preventable and potentially preventable through immunisation. A full review and discussion relating to the cost effectiveness of influenza and pneumococcal vaccination, including the role of the newer pneumococcal conjugate vaccines, was deemed to be outside the remit of this document.

Existing Department of Health guidelines are referred to (the Green book; see http://www.dh.gov.uk/en/Publichealth/ Healthprotection/Immunisation/Greenbook/DH_4097254).

Recommendations

- Department of Health guidelines in relation to influenza and pneumococcal immunisation of at-risk individuals should be followed. [C]

- All patients aged $>65$ years or at risk of invasive pneumococcal disease who are admitted with CAP and who have not previously received pneumococcal vaccine should receive 23 -valent pneumococcal polysaccharide vaccine (23-PPV) at convalescence in line with the Department of Health guidelines. [C]

\subsection{Smoking cessation}

Cigarette smoking, both active and passive, is a recognised independent risk factor for CAP. ${ }^{498} 499$ [Ib] [ib] Dose-response relationships with the current number of cigarettes smoked per day, pack-years of smoking and time since quitting have been demonstrated in relation to invasive pneumococcal disease. ${ }^{500}$ [III]

Recommendations

- Smoking cessation advice should be offered to all patients with CAP who are current smokers according to smoking cessation guidelines issued by the Health Education Authority. ${ }^{501}[\mathrm{~B}+]$

\section{SECTION 11 COMMITTEE MEMBERSHIP AND ACKNOWLEDGEMENTS}

11.1 Membership of the BTS Community Acquired Pneumonia Guidelines Committee and affiliations

Wei Shen Lim (Chairman), Consultant Respiratory Physician, Nottingham University Hospitals; Simon Baudouin, Senior Lecturer in Critical Care Medicine, Royal Victoria Infirmary and Intensive Care Society; Robert George, Director Respiratory and Systemic Infections Department, Health Protection Agency Centre for Infections, Colindale; Adam Hill, Consultant 
Respiratory Physician, Edinburgh Royal Infirmary; Conor Jamieson, Principal Pharmacist - Anti-infectives, Heart of England NHS Trust and British Society of Antimicrobial Chemotherapy; Ivan Le Jeune, Consultant in Acute Medicine, Nottingham University Hospitals and Society for Acute Medicine; John Macfarlane, Professor of Respiratory Medicine, University of Nottingham and Consultant Respiratory Physician, Nottingham University Hospitals; Robert Read, Professor in Infectious Diseases, University of Sheffield and British Infection Society; Helen Roberts, Specialist Registrar in Respiratory Medicine, Mid-Trent rotation, Nottingham University Hospitals; Mark Levy, General Practitioner, Royal College of General Practitioners and General Practice Airways Group (GPIAG); Mushtaq Wani, Health Care of the Elderly Consultant, Swansea NHS Trust and British Geriatrics Society; Mark Woodhead, Consultant Respiratory Physician, Manchester Royal Infirmary.

\subsection{Authorship of sections of the guidelines}

At least two clinical experts (a lead and a partner) were identified for each of the main topic areas and were responsible for the work of critically appraising the literature and preparing a main draft for the relevant section as described in section 1.8.

\section{Section leads and partners}

Incidence and mortality: H Roberts, WS Lim

Aetiology and epidemiology: M A Woodhead, R George

Clinical and radiological features: I Le Jeune, M Wani

General investigations: I Le Jeune, M Wani

Microbiological investigations: R George, W S Lim

Severity assessment: W S Lim, H Roberts

General management in hospital: A Hill, S Baudouin

Critical care issues: S Baudouin, A Hill

Antibiotic therapy: R Read, M A Woodhead, R George, J T Macfarlane

Complications and failure to improve: H Roberts, W S Lim

Primary care issues: M Levy, J T Macfarlane

\subsection{Acknowledgements}

The Guidelines Development Committee would like to thank many individuals and societies who have contributed to the development of this guideline. In particular, we thank Karen McClean, Sue Allen, Elizabeth Hendron and Natalie Middlemore for organisational, administrative and library support; Dr Richard Bendall, Dr John Bremner, Dr Graham Douglas, Professor Paul Edelstein, Professor Roger Finch, Rosalind Green, Dr Kieran Hand, Dr Elisabeth Ridgway, Dr Martin Springsklee, Dr Kate Templeton, the Department of Health Advisory Committee on Antimicrobial Resistance and Healthcare Associated Infection, Joint Committee on Vaccination and Immunisation, Scottish Clinical Virology Consultants Group and the UK Clinical Virology Network for constructive advice, comments and contributions received; Lisa Stirk, Information Officer at the Centre for Reviews and Dissemination, University of York for performing the literature searches; and Sally Welham, Deputy Chief Executive, British Thoracic Society for support and advice throughout the guideline development process.

\subsection{Declarations of interest}

The committee members fulfilled the requirements of the BTS regarding personal declaration of interests. Declaration of interest forms were updated annually by committee members and the contents submitted to the BTS Standards of Care Committee. These are available for inspection on request from the Chairman of this Committee.

Competing interests: None.

Provenance and peer review: Not commissioned; not externally peer reviewed.

\section{REFERENCES}

1. Macfarlane JT, Boswell T, Douglas G, et al. BTS guidelines on the management of community acquired pneumonia in adults. Thorax 2001;56(Suppl 4):1-64.

2. British Thoracic Society Pneumonia Guidelines Committee. BTS guidelines for the management of community acquired pneumonia in adults - 2004 update. 2004 http://www.brit-thoracic.org.uk/Portals/0/Clinical\%20Information/Pneumonia/ Guidelines/MACAPrevisedApr04.pdf (accessed 15 Dec 2008).

3. Macfarlane J. Lower respiratory tract infection and pneumonia in the community Semin Respir Infect 1999:14:151-62.

4. Macfarlane J. An overview of community acquired pneumonia with lessons learned from the British Thoracic Society Study. Semin Respir Infect 1994;9:153-65

5. Marrie TJ, Durant H, Yates L. Community-acquired pneumonia requiring hospitalization: 5-year prospective study. Rev Infect Dis 1989;11:586-99.

6. British Thoracic Society. Community-acquired pneumonia in adults in British hospitals in 1982-1983: a survey of aetiology, mortality, prognostic factors and outcome. $0 \mathrm{~J}$ Med 1987;62:195-220.

7. National Institute for Health and Clinical Excellence (NICE). National clinical guideline on management of chronic obstructive pulmonary disease in adults in primary and secondary care. Thorax 2004;51(Suppl 1):1-232.

8. British Thoracic Society Standards of Care Committee. Guidelines for the management of community acquired pneumonia in childhood. Thorax 2002;57(Suppl 1).

9. Department of Health and Health Protection Agency. Clostridium difficile infection. How to deal with the problem. Best Practice Guideline. 2008.

10. Owens RC Jr, Donskey CJ, Gaynes RP, et al. Antimicrobial-associated risk factors for Clostridium difficile infection. Clin Infect Dis 2008;46(Suppl 1):S19-31.

11. Tacconelli $\mathbf{E}$, De Angelis G, Cataldo MA, et al. Does antibiotic exposure increase the risk of methicillin-resistant Staphylococcus aureus (MRSA) isolation? A systematic review and meta-analysis. J Antimicrob Chemother 2008:61:26-38.

12. Trotter CL, Stuart JM, George R, et al. Increasing hospital admissions for pneumonia, England. Emerg Infect Dis 2008;14:727-33.

13. Woodhead M, Welch CA, Harrison DA, et al. Community-acquired pneumonia on the intensive care unit: secondary analysis of 17,869 cases in the ICNARC Case Mix Programme Database. Crit Care 2006;10(Suppl 2):S1.

14. Royal College of Physicians. Acute medical care. The right person, in the right setting - first time. Report of the Acute Medicine Task Force. London: Royal College of Physicians, 2007.

15. Department of Health. The NHS Plan: a plan for investment, a plan for reform. London: Department of Health, 2000

16. Mandell LA, Wunderink RG, Anzueto A, et al. Infectious Diseases Society of America/American Thoracic Society consensus guidelines on the management of community-acquired pneumonia in adults. Clin Infect Dis 2007:44(Suppl 2):S27-72.

17. Eccles M, Clapp Z, Grimshaw J, et al. North of England evidence based guidelines development project: methods of guideline development. BMJ 1996;312:760-2.

18. Sackett DL. Bias in analytic research. J Chronic Dis 1979:32:51-63.

19. Jaeschke R, Guyatt G, Sackett DL. Users' guides to the medical literature. III. How to use an article about a diagnostic test. A. Are the results of the study valid? Evidence-Based Medicine Working Group. JAMA 1994;271:389-91.

20. Scottish Intercollegiate Guidelines Network. Clinical guidelines: criteria for appraisal. Edinburgh: Royal College of Physicians of Edinburgh, 1995.

21. Eddy DM. A manual for assessing health practices and designing practice policies. Philadelphia: American College of Physicians, 1992.

22. Guyatt GH, Sackett DL, Sinclair JC, et al. Users' guides to the medical literature. IX A method for grading health care recommendations. Evidence-Based Medicine Working Group. JAMA 1995;274:1800-4.

23. Woodhead MA, Macfarlane JT, McCracken JS, et al. Prospective study of the aetiology and outcome of pneumonia in the community. Lancet 1987;1:671-4.

24. Jokinen C, Heiskanen $\mathrm{L}$, Juvonen $\mathrm{H}$, et al. Incidence of community-acquired pneumonia in the population of four municipalities in eastern Finland. Am J Epidemiol 1993:137:977-88

25. Foy HM, Cooney MK, Allan I, et al. Rates of pneumonia during influenza epidemics in Seattle, 1964 to 1975. JAMA 1979;241:253-8.

26. Macfarlane JT, Colville A, Guion A, et al. Prospective study of aetiology and outcome of adult lower-respiratory-tract infections in the community. Lancet 1993;341:511-4

27. Marrie TJ. Epidemiology of community-acquired pneumonia in the elderly. Semin Respir Infect 1990;5:260-8.

28. Lave JR, Fine MJ, Sankey SS, et al. Hospitalized pneumonia. Outcomes, treatment patterns, and costs in urban and rural areas. J Gen Intern Med 1996:11:415-21.

29. Marrie TJ, Carriere KC, Jin Y, et al. Factors associated with death among adults $<55$ years of age hospitalized for community-acquired pneumonia. Clin Infect Dis 2003;36:413-21.

30. Guest JF, Morris A. Community-acquired pneumonia: the annual cost to the National Health Service in the UK. Eur Respir J 1997:10:1530-4. 
31. Foy HM, Cooney MK, McMahan R, et al. Viral and mycoplasmal pneumonia in a prepaid medical care group during an eight-year period. Am J Epidemiol 1973;97:93-102.

32. Minogue MF, Coley CM, Fine MJ, et al. Patients hospitalized after initial outpatient treatment for community-acquired pneumonia. Ann Emerg Med 1998;31:376-80.

33. Torres A, Serra-Batlles J, Ferrer A, et al. Severe community-acquired pneumonia. Epidemiology and prognostic factors. Am Rev Respir Dis 1991;144:312-8.

34. Woodhead MA, Macfarlane JT, Rodgers FG, et al. Aetiology and outcome of severe community-acquired pneumonia. J Infect 1985;10:204-10.

35. White RJ, Blainey $A D$, Harrison $\mathrm{KJ}$, et al. Causes of pneumonia presenting to a district general hospital. Thorax 1981;36:566-70.

36. Macfarlane JT, Finch RG, Ward MJ, et al. Hospital study of adult communityacquired pneumonia. Lancet 1982;2:255-8.

37. Lim WS, Macfarlane JT, Boswell TC, et al. Study of community acquired pneumonia aetiology (SCAPA) in adults admitted to hospital: implications for management guidelines. Thorax 2001;56:296-301.

38. Karalus NC, Cursons RT, Leng RA, et al. Community acquired pneumonia: aetiology and prognostic index evaluation. Thorax 1991;46:413-8.

39. Lim I, Shaw DR, Stanley DP, et al. A prospective hospital study of the aetiology of community-acquired pneumonia. Med J Aust 1989;151:87-91.

40. Mortensen EM, Kapoor WN, Chang C-CH, et al. Assessment of mortality after long-term follow-up of patients with community-acquired pneumonia. Clin Infect Dis 2003;37:1617-24

41. Yende S, Angus DC, Ali IS, et al. Influence of comorbid conditions on long-term mortality after pneumonia in older people. J Am Geriatr Soc 2007;55:518-25.

42. British Thoracic Society Research Committee and Public Health Laboratory Service. The aetiology, management and outcome of severe community-acquired pneumonia on the intensive care unit. Respir Med 1992;86:7-13.

43. Alkhayer $\mathbf{M}$, Jenkins PF, Harrison BD. The outcome of community acquired pneumonia treated on the intensive care unit. Respir Med 1990;84:13-6.

44. Moine P, Vercken JB, Chevret $\mathrm{S}$, et al. Severe community-acquired pneumonia. Etiology, epidemiology, and prognosis factors. French Study Group for CommunityAcquired Pneumonia in the Intensive Care Unit. Chest 1994;105:1487-95.

45. Niederman MS, McCombs JS, Unger AN, et al. The cost of treating communityacquired pneumonia. Clin Ther 1998;20:820-37.

46. Gilbert K, Gleason PP, Singer DE, et al. Variations in antimicrobial use and cost in more than 2,000 patients with community-acquired pneumonia. Am J Med 1998; 104:17-27.

47. Howard LSGE, Sillis M, Pasteur MC, et al. Microbiological profile of communityacquired pneumonia in adults over the last 20 years. J Infect 2005;50:107-13.

48. Lagerstrom F, Bader M, Foldevi M, et al. Microbiological etiology in clinically diagnosed community-acquired pneumonia in primary care in Orebro, Sweden. Clin Microbiol Infect 2003;9:645-52

49. Ruiz M, Ewig S, Marcos MA, et al. Etiology of community-acquired pneumonia: impact of age, comorbidity, and severity. Am J Respir Crit Care Med 1999;160:397-405

50. de Roux A, Ewig S, Garcia E, et al. Mixed community-acquired pneumonia in hospitalised patients. Eur Respir J 2006;27:795-800.

51. Gutierrez F, Masia M, Rodriguez JC, et al. Epidemiology of community-acquired pneumonia in adult patients at the dawn of the 21st century: a prospective study on the Mediterranean coast of Spain. Clin Microbiol Infect 2005;11:788-800.

52. Fernandez Alvarez R, Suarez Toste I, Rubinos Cuadrado G, et al. Communityacquired pneumonia: aetiologic changes in a limited geographic area. An 11-year prospective study. Eur J Clin Microbiol Infect Dis 2007;26:495-9.

53. Arnold FW, Summersgill JT, Lajoie AS, et al. A worldwide perspective of atypical pathogens in community-acquired pneumonia. Am J Respir Crit Care Med 2007:175:1086-93.

54. Ngeow Y-F, Suwanjutha S, Chantarojanasriri T, et al. An Asian study on the prevalence of atypical respiratory pathogens in community-acquired pneumonia. Int $\mathrm{J}$ Infect Dis 2005;9:144-53.

55. Diaz A, Barria P, Niederman M, et al. Etiology of community-acquired pneumonia in hospitalized patients in Chile: the increasing prevalence of respiratory viruses among classic pathogens. Chest 2007:131:779-87.

56. Matute AJ, Brouwer WP, Hak E, et al. Aetiology and resistance patterns of community-acquired pneumonia in Leon, Nicaragua. Int $J$ Antimicrob Agents 2006;28:423-7.

57. Nagalingam NA, Adesiyun AA, Swanston WH, et al. Seroprevalence of Legionella pneumophila in pneumonia patients in four major hospitals in Trinidad and Tobago. West Indian Med J 2005;54:375-8.

58. Chedid MBF, llha DdO, Chedid MF, et al. Community-acquired pneumonia by Legionella pneumophila serogroups 1-6 in Brazil. Respir Med 2005;99:966-75.

59. Marrie TJ, Peeling RW, Reid T, et al. Chlamydia species as a cause of communityacquired pneumonia in Canada. Eur Respir $J$ 2003;21:779-84.

60. Valdivia L, Nix D, Wright $\mathrm{M}$, et al. Coccidioidomycosis as a common cause of community-acquired pneumonia [correction appears in Emerg Infect Dis 2006;12:1307]. Emerg Infect Dis 2006;12:958-62.

61. Wattanathum A, Chaoprasong C, Nunthapisud P, et al. Community-acquired pneumonia in southeast Asia: the microbial differences between ambulatory and hospitalized patients. Chest 2003;123:1512-9.

62. Bansal S, Kashyap S, Pal LS, et al. Clinical and bacteriological profile of community acquired pneumonia in Shimla, Himachal Pradesh. Indian J Chest Dis Allied Sci 2004:46:17-22.
63. Reechaipichitkul W, Lulitanond V, Sawanyawisuth K, et al. Etiologies and treatment outcomes for out-patients with community-acquired pneumonia (CAP) at Srinagarind Hospital, Khon Kaen, Thailand. Southeast Asian J Trop Med Publ Health 2005:36:1261-7.

64. Yen M-Y, Hu B-S, Chen Y-S, et al. A prospective etiologic study of communityacquired pneumonia in Taiwan. J Formosan Med Assoc 2005;104:724-30.

65. Huang HH, Zhang YY, Xiu QY, et al. Community-acquired pneumonia in Shanghai, China: microbial etiology and implications for empirical therapy in a prospective study of 389 patients. Eur J Clin Microbiol Infect Dis 2006;25:369-74.

66. Saito A, Kohno S, Matsushima T, et al. Prospective multicenter study of the causative organisms of community-acquired pneumonia in adults in Japan. $J$ Infect Chemother 2006:12:63-9

67. Lauderdale T-L, Chang F-Y, Ben R-J, et al. Etiology of community acquired pneumonia among adult patients requiring hospitalization in Taiwan. Respir Med 2005:99:1079-86.

68. Paganin F, Lilienthal F, Bourdin A, et al. Severe community-acquired pneumonia: assessment of microbial aetiology as mortality factor. Eur Respir $\boldsymbol{J}$ 2004;24:779-85.

69. Venkatesan P, Gladman J, Macfarlane JT, et al. A hospital study of community acquired pneumonia in the elderly. Thorax 1990;45:254-8.

70. Ausina V, Coll P, Sambeat M, et al. Prospective study on the etiology of community-acquired pneumonia in children and adults in Spain. Eur J Clin Microbiol Infect Dis 1988;7:342-7.

71. Logroscino CD, Penza 0, Locicero S, et al. Community-acquired pneumonia in adults: a multicentric observational AIPO study. Monaldi Arch Chest Dis 1999;54:11-7.

72. Leroy $\mathbf{0}$, Vandenbussche $\mathrm{C}$, Coffinier $\mathrm{C}$, et al. Community-acquired aspiration pneumonia in intensive care units. Epidemiological and prognosis data. Am J Respir Crit Care Med 1997;156:1922-9.

73. Fernandez-Sabe N, Carratala J, Roson B, et al. Community-acquired pneumonia in very elderly patients: causative organisms, clinical characteristics, and outcomes. Medicine 2003;82:159-69.

74. Ostergaard L, Andersen PL. Etiology of community-acquired pneumonia. Evaluation by transtracheal aspiration, blood culture, or serology. Chest 1993;104:1400-7.

75. Torres A, Dorca J, Zalacaín R, et al. Community-acquired pneumonia in chronic obstructive pulmonary disease: a Spanish multicenter study. Am J Respir Crit Care Med 1996;154:1456-61.

76. Marrie TJ. Bacteraemic pneumococcal pneumonia: a continuously evolving disease. J Infect 1992;24:247-55.

77. Marrie TJ, Durant H, Kwan C. Nursing home-acquired pneumonia. A case-control study. J Am Geriatr Soc 1986;34:697-702

78. Garb JL, Brown RB, Garb JR, et al. Differences in etiology of pneumonias in nursing home and community patients. JAMA 1978;240:2169-72.

79. Drinka PJ, Gauerke C, Voeks S, et al. Pneumonia in a nursing home. J Gen Intern Med 1994;9:650-2.

80. Lim WS, Macfarlane JT. A prospective comparison of nursing home acquired pneumonia with community acquired pneumonia. Eur Respir J 2001;18:362-8.

81. Carratala J, Mykietiuk A, Fernandez-Sabe N, et al. Health care-associated pneumonia requiring hospital admission: epidemiology, antibiotic therapy, and clinical outcomes. Arch Intern Med 2007;167:1393-9.

82. Sopena N, Sabrià-Leal M, Pedro-Botet ML, et al. Comparative study of the clinical presentation of Legionella pneumonia and other community-acquired pneumonias. Chest 1998;113:1195-200.

83. de Roux A, Cavalcanti M, Marcos MA, et al. Impact of alcohol abuse in the etiology and severity of community-acquired pneumonia. Chest 2006;129:1219-25.

84. Stout JE, Yu VL. Legionellosis. N Engl J Med 1997;337:682-7.

85. Mier L, Dreyfuss D, Darchy B, et al. Is penicillin $\mathrm{G}$ an adequate initial treatment for aspiration pneumonia? A prospective evaluation using a protected specimen brush and quantitative cultures. Intensive Care Med 1993;19:279-84.

86. de Roux A, Marcos MA, Garcia E, et al. Viral community-acquired pneumonia in nonimmunocompromised adults. Chest 2004;125:1343-51.

87. Kim PE, Musher DM, Glezen WP, et al. Association of invasive pneumococcal disease with season, atmospheric conditions, air pollution, and the isolation of respiratory viruses. Clin Infect Dis 1996;22:100-6.

88. Mercat A, Nguyen J, Dautzenberg B. An outbreak of pneumococcal pneumonia in two men's shelters. Chest 1991;99:147-51.

89. Naik FC, Ricketts KD, Harrison TG, et al. Legionnaires' disease in England and Wales (1995-2005). Health Protection Report 2008. 2008.

90. Newton LH, Joseph CA, Hutchinson EJ, et al. Legionnaires' disease surveillance: England and Wales, 1995. Commun Dis Rep CDR Rev 1996;6:R151-5.

91. Joseph CA, Harrison TG, llijic-Car D, et al. Legionnaires' disease in residents of England and Wales: 1998. Commun Dis Public Health 1999;2:280-4.

92. Joseph CA, Harrison TG, llijic-Car D, et al. Legionnaires' disease in residents of England and Wales: 1996. Commun Dis Rep CDR Rev 1997;7:R153-9.

93. Saikku $\mathbf{P}$, Wang SP, Kleemola $\mathrm{M}$, et al. An epidemic of mild pneumonia due to an unusual strain of Chlamydia psittaci. J Infect Dis 1985;151:832-9.

94. Kleemola M, Saikku P, Visakorpi R, et al. Epidemics of pneumonia caused by TWAR, a new Chlamydia organism, in military trainees in Finland. J Infect Dis 1988;157:230-6.

95. Troy CJ, Peeling RW, Ellis AG, et al. Chlamydia pneumoniae as a new source of infectious outbreaks in nursing homes. JAMA 1997;277:1214-8.

96. Wellinghausen N, Straube E, Freidank $\mathrm{H}$, et al. Low prevalence of Chlamydia pneumoniae in adults with community-acquired pneumonia. Int J Med Microbiol 2006;296:485-91. 
97. Steinhoff D, Lode H, Ruckdeschel G, et al. Chlamydia pneumoniae as a cause of community-acquired pneumonia in hospitalized patients in Berlin. Clin Infect Dis 1996;22:958-64.

98. Lieberman D, Ben-Yaakov M, Lazarovich Z, et al. Chlamydia pneumoniae community-acquired pneumonia: a review of 62 hospitalized adult patients. Infection 1996;24:109-14.

99. Kauppinen MT, Saikku P, Kujala P, et al. Clinical picture of community-acquired Chlamydia pneumoniae pneumonia requiring hospital treatment: a comparison between chlamydial and pneumococcal pneumonia. Thorax 1996;51:185-9.

100. Wreghitt T. Chlamydial infection of the respiratory tract. Commun Dis Rep CDR Rev 1993;3:R119-24.

101. Pebody RG, Wall PG, Ryan MJ, et al. Epidemiological features of Coxiella burnetii infection in England and Wales: 1984 to 1994. Commun Dis Rep CDR Rev 1996;6:R128-32.

102. McNabb WR, Shanson DC, Williams TD, et al. Adult community-acquired pneumonia in central London. J R Soc Med 1984;77:550-5.

103. Hirani NA, Macfarlane JT. Impact of management guidelines on the outcome of severe community acquired pneumonia. Thorax 1997:52:17-21.

104. Rotas M, McCalla S, Liu C, et al. Methicillin-resistant Staphylococcus aureus necrotizing pneumonia arising from an infected episiotomy site. Obstet Gynecol 2007;109:533-6.

105. Asnis D, Haralambou G, Tawiah P, et al. Methicillin-resistant Staphylococcus aureus necrotizing pneumonia arising from an infected episiotomy site. Obstet Gynecol 2007;110:188

106. Garnier F, Tristan A, Francois B, et al. Pneumonia and new methicillin-resistant Staphylococcus aureus clone. Emerg Infect Dis 2006;12:498-500.

107. Nimmo GR, Playford EG. Community-acquired MRSA bacteraemia: four additional cases including one associated with severe pneumonia. Med J Aust 2003;178:245.

108. Peleg AY, Munckhof WJ. Fatal necrotising pneumonia due to community-acquired methicillin-resistant Staphylococcus aureus (MRSA). Med J Aust 2004;181:228-9.

109. Risson DC, O'Connor ED, Guard RW, et al. A fatal case of necrotising pneumonia due to community-associated methicillin-resistant Staphylococcus aureus. Med J Aust 2007;186:479-80.

110. Vayalumkal JV, Whittingham $\mathrm{H}$, Vanderkooi 0 , et al. Necrotizing pneumonia and septic shock: suspecting CA-MRSA in patients presenting to Canadian emergency departments. CJEM Can J Emerg Med Care 2007;9:300-3.

111. Monaco M, Antonucci R, Palange P, et al. Methicillin-resistant Staphylococcus aureus necrotizing pneumonia. Emerg Infect Dis 2005;11:1647-8.

112. Torell E, Molin D, Tano E, et al. Community-acquired pneumonia and bacteraemia in a healthy young woman caused by methicillin-resistant Staphylococcus aureus (MRSA) carrying the genes encoding Panton-Valentine leukocidin (PVL). Scand J Infect Dis 2005;37:902-4.

113. Soderquist B, Berglund C, Stralin K. Community-acquired pneumonia and bacteremia caused by an unusual methicillin-resistant Staphylococcus aureus (MRSA) strain with sequence type 36, staphylococcal cassette chromosome mec type IV and Panton-Valentine leukocidin genes. Eur J Clin Microbiol Infect Dis 2006;25:604-6.

114. Magira EE, Zervakis D, Routsi C, et al. Community-acquired methicillin-resistant Staphylococcus aureus carrying Panton-Valentine leukocidin genes: a lethal cause of pneumonia in an adult immunocompetent patient. Scand J Infect Dis 2007;39:466-9.

115. Klein JL, Petrovic Z, Treacher D, et al. Severe community-acquired pneumonia caused by Panton-Valentine leukocidin-positive Staphylococcus aureus: first reported case in the United Kingdom. Intensive Care Med 2003;29:1399.

116. Honarpour N, Mao JT. A Case of fatal community-acquired necrotizing pneumonia caused by panton-valentine leukocidin positive methicillin-sensitive Staphylococcus aureus. Curr Respir Med Rev 2007;3:49-51.

117. Beilouny B, Ciupea A, Eloy C, et al. Fatal community-acquired pneumonia due to Staphylococcus aureus carrying Panton-Valentine leukocidin genes after a stay in Africa. Intensive Care Med 2008;34:388-9.

118. Baldwin LN, Lowe AD. Panton-Valentine Leukocidin associated with community acquired methicillin resistant Staphylococcus aureus: a case report and review of interim guidelines. Anaesthesia 2008;63:764-6.

119. Francis JS, Doherty MC, Lopatin U, et al. Severe community-onset pneumonia in healthy adults caused by methicillin-resistant Staphylococcus aureus carrying the Panton-Valentine leukocidin genes. Clin Infect Dis 2005;40:100-7.

120. Hageman JC, Uyeki TM, Francis JS, et al. Severe community-acquired pneumonia due to Staphylococcus aureus, 2003-04 influenza season. Emerg Infect Dis 2006;12:894-9.

121. Chua AP, Lee KH. Fatal bacteraemic pneumonia due to community-acquired methicillin-resistant Staphylococcus aureus. Singapore Med J 2006;47:546-8.

122. Anon. Severe methicillin-resistant Staphylococcus aureus community-acquired pneumonia associated with influenza - Louisiana and Georgia, December 2006January 2007. MMWR - Morbidity \& Mortality Weekly Report 2007;56:325-9.

123. Hyvernat H, Pulcini C, Carles D, et al. Fatal Staphylococcus aureus haemorrhagic pneumonia producing Panton-Valentine leucocidin. Scand J Infect Dis 2007;39:183-5.

124. Balis E, Diacaki $C$, Tselioti $P$, et al. Community-acquired pneumonia and bacteremia due to methicillin-resistant Staphylococcus aureus carrying Panton-Valentineleukocidin gene in Greece: two case reports and literature review. J Chemother 2007; 19:703-8.

125. Hutchinson EJ, Joseph CA, Zambon M, et al. Influenza surveillance in England and Wales: October 1995 to June 1996. Commun Dis Rep CDR Rev 1996;6:R163-9.
126. Connolly AM, Salmon RL, Lervy B, et al. What are the complications of influenza and can they be prevented? Experience from the 1989 epidemic of H3N2 influenza A in general practice. BMJ 1993;306:1452-4.

127. Farr BM, Kaiser DL, Harrison BD, et al. Prediction of microbial aetiology at admission to hospital for pneumonia from the presenting clinical features. British Thoracic Society Pneumonia Research Subcommittee. Thorax 1989;44:1031-5.

128. Fang GD, Fine M, Orloff J, et al. New and emerging etiologies for communityacquired pneumonia with implications for therapy. A prospective multicenter study of 359 cases. Medicine (Baltimore) 1990;69:307-16.

129. Riquelme R, Torres A, El-Ebiary $\mathrm{M}$, et al. Community-acquired pneumonia in the elderly: a multivariate analysis of risk and prognostic factors. Am J Respir Crit Care Med 1996;154:1450-5.

130. Bohte R, Hermans J, van den Broek PJ. Early recognition of Streptococcus pneumoniae in patients with community-acquired pneumonia. Eur J Clin Microbiol Infect Dis 1996;15:201-5

131. Helms CM, Wintermeyer LA, Zeitler RR, et al. An outbreak of community-acquired Legionnaires' disease pneumonia. Am J Public Health 1984;74:835-6.

132. Granados A, Podzamczer D, Gudiol F, et al. Pneumonia due to Legionella pneumophila and pneumococcal pneumonia: similarities and differences on presentation. Eur Respir J 1989;2:130-4.

133. Fernandez-Sabe N, Roson B, Carratala J, et al. Clinical diagnosis of Legionella pneumonia revisited: evaluation of the Community-Based Pneumonia Incidence Study Group scoring system. Clin Infect Dis 2003;37:483-9.

134. Lieberman D, Porath A, Schlaeffer F, et al. Legionella species community-acquired pneumonia. A review of 56 hospitalized adult patients. Chest 1996;109:1243-9.

135. Falcó V, Fernández de Sevilla T, Alegre J, et al. Legionella pneumophila. A cause of severe community-acquired pneumonia. Chest 1991;100:1007-11.

136. Helms CM, Viner JP, Sturm RH, et al. Comparative features of pneumococcal, mycoplasmal, and Legionnaires' disease pneumonias. Ann Intern Med 1979:90:543-7.

137. Marrie TJ. Mycoplasma pneumoniae pneumonia requiring hospitalization, with emphasis on infection in the elderly. Arch Intern Med 1993;153:488-94.

138. Puljiz I, Kuzman I, Dakovic-Rode 0, et al. Chlamydia pneumoniae and Mycoplasma pneumoniae pneumonia: comparison of clinical, epidemiological characteristics and laboratory profiles. Epidemiol Infect 2006;134:548-55.

139. File TM Jr, Plouffe JF Jr, Breiman RF, et al. Clinical characteristics of Chlamydia pneumoniae infection as the sole cause of community-acquired pneumonia. Clin Infect Dis 1999;29:426-8.

140. Lieberman D, Boldur I, Manor E, et al. Q-fever pneumonia in the Negev region of Israel: a review of 20 patients hospitalised over a period of one year. J Infect 1995;30:135-40.

141. Goyette $\mathbf{M}$, Bouchard J, Poirier A, et al. Importance of $\mathbf{Q}$ fever in community acquired pneumonia. Can J Infect Dis 1996;7:370-3.

142. Sobradillo V, Ansola P, Baranda F, et al. 0 fever pneumonia: a review of 164 community-acquired cases in the Basque country. Eur Respir J 1989;2:263-6.

143. Caron F, Meurice JC, Ingrand P, et al. Acute 0 fever pneumonia: a review of 80 hospitalized patients. Chest 1998;114:808-13.

144. Feldman C, Kallenbach JM, Levy H, et al. Comparison of bacteraemic communityacquired lobar pneumonia due to Streptococcus pneumoniae and Klebsiella pneumoniae in an intensive care unit. Respiration 1991;58:265-70.

145. Rudin ML, Michael JR, Huxley EJ. Community-acquired acinetobacter pneumonia Am J Med 1979;67:39-43.

146. Henderson A, Wall D. Streptococcus milleri liver abscess presenting as fulminant pneumonia. Aust N Z J Surg 1993;63:237-40.

147. Goolam MA, Feldman C, Smith C, et al. Does primary Streptococcus viridans pneumonia exist? S Afr Med J 1992;82:432-4.

148. Marrie TJ. Pneumonia in the elderly. Curr Opin Pulm Med 1996;2:192-7.

149. Metlay JP, Schulz R, Li YH, et al. Influence of age on symptoms at presentation in patients with community-acquired pneumonia. Arch Intern Med 1997;157:1453-9.

150. Riquelme R, Torres A, el-Ebiary $M$, et al. Community-acquired pneumonia in the elderly. Clinical and nutritional aspects. Am J Respir Crit Care Med 1997;156:1908-14.

151. Marrie TJ, Haldane EV, Faulkner RS, et al. Community-acquired pneumonia requiring hospitalization. Is it different in the elderly? J Am Geriatr Soc 1985;33:671-80.

152. Kikuchi R, Watabe N, Konno T, et al. High incidence of silent aspiration in elderly patients with community-acquired pneumonia. Am J Respir Crit Care Med 1994;150:251-3

153. Marrie TJ, Blanchard W. A comparison of nursing home-acquired pneumonia patients with patients with community-acquired pneumonia and nursing home patients without pneumonia. J Am Geriatr Soc 1997;45:50-5.

154. Reza Shariatzadeh M, Huang J0, Marrie TJ. Differences in the features of aspiration pneumonia according to site of acquisition: community or continuing care facility. J Am Geriatr Soc 2006;54:296-302.

155. Bartlett JG, Gorbach SL, Finegold SM. The bacteriology of aspiration pneumonia Am J Med 1974:56:202-7.

156. Marik PE, Careau P. The role of anaerobes in patients with ventilator-associated pneumonia and aspiration pneumonia: a prospective study. Chest 1999;115:178-83.

157. El-Solh AA, Pietrantoni C, Bhat A, et al. Microbiology of severe aspiration pneumonia in institutionalized elderly. Am J Respir Crit Care Med 2003;167:1650-4.

158. Ott SR, Allewelt M, Lorenz J, et al. Moxifloxacin vs ampicillin/sulbactam in aspiration pneumonia and primary lung abscess. Infection 2008;36:23-30. 
159. Metlay JP, Kapoor WN, Fine MJ. Does this patient have community-acquired pneumonia? Diagnosing pneumonia by history and physical examination. JAMA 1997;278:1440-5

160. Metlay JP, Fine MJ. Testing strategies in the initial management of patients with community-acquired pneumonia. Ann Intern Med 2003;138:109-18.

161. Spiteri MA, Cook DG, Clarke SW. Reliability of eliciting physical signs in examination of the chest. Lancet 1988:1:873-5.

162. Melbye H, Straume B, Aasebø U, et al. The diagnosis of adult pneumonia in general practice. The diagnostic value of history, physical examination and some blood tests. Scand J Prim Health Care 1988:6:111-7.

163. Diehr P, Wood RW, Bushyhead J, et al. Prediction of pneumonia in outpatients with acute cough—a statistical approach. J Chronic Dis 1984;37:215-25.

164. O'Brien WT Sr, Rohweder DA, Lattin GE Jr, et al. Clinical indicators of radiographic findings in patients with suspected community-acquired pneumonia: who needs a chest x-ray? [correction appears in J Am Coll Radiol 2006;3:A4]. J Am Coll Radiol 2006;3:703-6.

165. Macfarlane J, Holmes W, Gard P, et al. Prospective study of the incidence, aetiology and outcome of adult lower respiratory tract illness in the community. Thorax 2001;56:109-14

166. Macfarlane JT, Miller AC, Roderick Smith WH, et al. Comparative radiographic features of community acquired Legionnaires' disease, pneumococcal pneumonia, mycoplasma pneumonia, and psittacosis. Thorax 1984;39:28-33.

167. Kauppinen MT, Lähde S, Syrjälä H. Roentgenographic findings of pneumonia caused by Chlamydia pneumoniae. A comparison with streptococcus pneumonia. Arch Intern Med 1996;156:1851-6.

168. Gikas A, Kofteridis D, Bouros D, et al. 0 fever pneumonia: appearance on chest radiographs. Radiology 1999;210:339-43.

169. Marrie TJ, Peeling RW, Fine MJ, et al. Ambulatory patients with communityacquired pneumonia: the frequency of atypical agents and clinical course. Am J Med 1996;101:508-15.

170. Macfarlane J, Rose D. Radiographic features of staphylococcal pneumonia in adults and children. Thorax 1996;51:539-40.

171. Korvick JA, Hackett AK, Yu VL, et al. Klebsiella pneumonia in the modern era: clinicoradiographic correlations. South Med J 1991;84:200-4.

172. Syrjälä H, Broas M, Suramo I, et al. High-resolution computed tomography for the diagnosis of community-acquired pneumonia. Clin Infect Dis 1998;27:358-63.

173. Reittner $\mathbf{P}$, Müller NL, Heyneman L, et al. Mycoplasma pneumoniae pneumonia: radiographic and high-resolution CT features in 28 patients. AJR Am J Roentgenol 2000;174:37-41.

174. Beall DP, Scott WW Jr, Kuhlman JE, et al. Utilization of computed tomography in patients hospitalized with community-acquired pneumonia. Md Med J 1998:47:182-7.

175. Tanaka N, Matsumoto T, Kuramitsu T, et al. High resolution CT findings in community-acquired pneumonia. J Comput Assist Tomogr 1996:20:600-8.

176. Mittl RL Jr, Schwab RJ, Duchin JS, et al. Radiographic resolution of communityacquired pneumonia. Am J Respir Crit Care Med 1994;149(3 Pt 1):630-5.

177. El Solh AA, Aquilina AT, Gunen H, et al. Radiographic resolution of communityacquired bacterial pneumonia in the elderly. J Am Geriatr Soc 2004;52:224-9.

178. Bruns AHW, Oosterheert JJ, Prokop M, et al. Patterns of resolution of chest radiograph abnormalities in adults hospitalized with severe community-acquired pneumonia. Clin Infect Dis 2007;45:983-91.

179. Jay SJ, Johanson WG Jr, Pierce AK. The radiographic resolution of Streptococcus pneumoniae pneumonia. N Engl J Med 1975;293:798-801.

180. Granet KM, Wallach SL, Horvath K, et al. Chest radiographs in patients with community-acquired pneumonia. N J Med 1996:93:37-41.

181. Holmberg H, Kragsbjerg P. Association of pneumonia and lung cancer: the value of convalescent chest radiography and follow-up. Scand J Infect Dis 1993;25:93-100.

182. Gibson SP, Weir DC, Burge PS. A prospective audit of the value of fibre optic bronchoscopy in adults admitted with community acquired pneumonia. Respir Med 1993;87:105-9.

183. British Thoracic Society. BTS guideline for emergency oxygen use in adult patients. Thorax 2008;63(Suppl 6):vi1-68.

184. Holmberg H, Bodin L, Jönsson I, et al. Rapid aetiological diagnosis of pneumonia based on routine laboratory features. Scand J Infect Dis 1990;22:537-45.

185. Castro-Guardiola A, Armengou-Arxe A, Viejo-Rodriguez A, et al. Differential diagnosis between community-acquired pneumonia and non-pneumonia diseases of the chest in the emergency ward. Eur J Intern Med 2000;11:334-9.

186. Smith RP, Lipworth BJ, Cree IA, et al. C-reactive protein. A clinical marker in community-acquired pneumonia. Chest 1995:108:1288-91.

187. Hansson LO, Hedlund JU, Ortqvist AB. Sequential changes of inflammatory and nutritional markers in patients with community-acquired pneumonia. Scand J Clin Lab Invest 1997;57:111-8.

188. Flanders SA, Stein J, Shochat G, et al. Performance of a bedside C-reactive protein test in the diagnosis of community-acquired pneumonia in adults with acute cough. Am J Med 2004;116:529-35.

189. Ortqvist A, Hedlund J, Wretlind B, et al. Diagnostic and prognostic value of interleukin-6 and C-reactive protein in community-acquired pneumonia. Scand J Infect Dis 1995;27:457-62

190. Garcia Vazquez E, Martinez JA, Mensa J, et al. C-reactive protein levels in community-acquired pneumonia. Eur Respir J 2003;21:702-5.

191. Sanyal S, Smith PR, Saha AC, et al. Initial microbiologic studies did not affect outcome in adults hospitalized with community-acquired pneumonia. Am J Respir Crit Care Med 1999;160:346-8.
192. Menéndez R, Córdoba J, de La Cuadra $P$, et al. Value of the polymerase chain reaction assay in noninvasive respiratory samples for diagnosis of communityacquired pneumonia. Am J Respir Crit Care Med 1999;159:1868-73.

193. Ewig S, Bauer T, Hasper $\mathrm{E}$, et al. Value of routine microbial investigation in community-acquired pneumonia. Can J Infect Dis 1996;63:169.

194. Woodhead MA, Arrowsmith J, Chamberlain-Webber R, et al. The value of routine microbial investigation in community-acquired pneumonia. Respir Med 1991;85:313-7.

195. van der Eerden MM, Vlaspolder F, de Graaff CS, et al. Value of intensive diagnostic microbiological investigation in low- and high-risk patients with community-acquired pneumonia. Eur J Clin Microbiol Infect Dis 2005;24:241-9.

196. Hohenthal U, Vainionpaa R, Meurman 0, et al. Aetiological diagnosis of community acquired pneumonia: utility of rapid microbiological methods with respect to disease severity. Scand J Infect Dis 2008;40:131-8.

197. Templeton KE, Scheltinga SA, van den Eeden WCJFM, et al. Improved diagnosis of the etiology of community-acquired pneumonia with real-time polymerase chain reaction. Clin Infect Dis 2005:41:345-51.

198. Theerthakarai R, El-Halees W, Ismail M, et al. Nonvalue of the initial microbiological studies in the management of nonsevere community-acquired pneumonia. Chest 2001;119:181-4.

199. Ewig S, Schlochtermeier M, Goke N, et al. Applying sputum as a diagnostic tool in pneumonia: limited yield, minimal impact on treatment decisions. Chest 2002;121:1486-92.

200. Waterer GW, Wunderink RG. The influence of the severity of community-acquired pneumonia on the usefulness of blood cultures. Respir Med 2001;95:78-82.

201. Campbell SG, Marrie TJ, Anstey R, et al. The contribution of blood cultures to the clinical management of adult patients admitted to the hospital with communityacquired pneumonia: a prospective observational study. Chest 2003;123:1142-50.

202. Woodhead M, Macfarlane J. Local antibiotic guidelines for adult communityacquired pneumonia (CAP): a survey of UK hospital practice in 1999. J Antimicrob Chemother 2000;46:141-3.

203. Chambers DC, Waterer GW. Are blood cultures necessary in community-acquired pneumonia? Clin Pulmon Med 2005;12:146-52.

204. Benenson RS, Kepner AM, Pyle DN 2nd, et al. Selective use of blood cultures in emergency department pneumonia patients. J Emerg Med 2007;33:1-8.

205. Ramanujam P, Rathlev NK. Blood cultures do not change management in hospitalized patients with community-acquired pneumonia. Acad Emerg Med 2006;13:740-5

206. Corbo J, Friedman B, Bijur P, et al. Limited usefulness of initial blood cultures in community acquired pneumonia. Emerg Med J 2004;21:446-8.

207. Bartlett JG, Dowell SF, Mandell LA, et al. Practice guidelines for the management of community-acquired pneumonia in adults. Infectious Diseases Society of America. Clin Infect Dis 2000;31:347-82

208. Taylor EL, Marrie TJ, Fine MJ, et al. Observations from a multicentre study on the use of the sputum specimen in patients hospitalized with community-acquired pneumonia. Can J Infect Dis 1999;10:39-46.

209. Reed WW, Byrd GS, Gates RH Jr, et al. Sputum gram's stain in community-acquired pneumococcal pneumonia. A meta-analysis. West J Med 1996;165:197-204.

210. Rein MF, Gwaltney JM Jr, O'Brien WM, et al. Accuracy of Gram's stain in identifying pneumococci in sputum. JAMA 1978;239:2671-3.

211. Garcia-Vazquez $\mathbf{E}$, Marcos MA, Mensa J, et al. Assessment of the usefulness of sputum culture for diagnosis of community-acquired pneumonia using the PORT predictive scoring system. Arch Intern Med 2004;164:1807-11.

212. Miyashita N, Shimizu H, Ouchi K, et al. Assessment of the usefulness of sputum Gram stain and culture for diagnosis of community-acquired pneumonia requiring hospitalization. Med Sci Monit 2008;14:CR171-6.

213. Fine MJ, Orloff JJ, Rihs JD, et al. Evaluation of housestaff physicians' preparation and interpretation of sputum Gram stains for community-acquired pneumonia. J Gen Intern Med 1991;6:189-98.

214. Croce MA, Fabian TC, Waddle-Smith L, et al. Utility of Gram's stain and efficacy of quantitative cultures for posttraumatic pneumonia: a prospective study. Ann Surg 1998;227:743-55

215. Roberts ME, Macfarlane JT, George RC, et al. Microbiology investigations in community acquired pneumonia-most laboratories in England and Wales do not offer all those recommended in the British Thoracic Society guideline. J Infect 2008; 56:291-4.

216. Venkatesan P, Macfarlane JT. Role of pneumococcal antigen in the diagnosis of pneumococcal pneumonia. Thorax 1992;47:329-31.

217. Murdoch DR, Laing RT, Mills GD, et al. Evaluation of a rapid immunochromatographic test for detection of Streptococcus pneumoniae antigen in urine samples from adults with community-acquired pneumonia. J Clin Microbiol 2001;39:3495-8.

218. Andreo F, Dominguez J, Ruiz J, et al. Impact of rapid urine antigen tests to determine the etiology of community-acquired pneumonia in adults. Respiratory Medicine 2006;100:884-91

219. Briones ML, Blanquer J, Ferrando D, et al. Assessment of analysis of urinary pneumococcal antigen by immunochromatography for etiologic diagnosis of community-acquired pneumonia in adults. Clin Vaccine Immunol 2006;13:1092-7.

220. Gutierrez F, Masia M, Rodriguez JC et al. Evaluation of the immunochromatographic Binax NOW assay for detection of Streptococcus pneumoniae urinary antigen in a prospective study of community-acquired pneumonia in Spain. Clin Infect Dis 2003;36:286-92. 
221. Kobashi Y, Yoshida K, Miyashita N, et al. Evaluating the use of a Streptococcus pneumoniae urinary antigen detection kit for the management of communityacquired pneumonia in Japan. Respiration 2007;74:387-93.

222. Lasocki S, Scanvic A, Le Turdu F, et al. Evaluation of the Binax NOW Streptococcus pneumoniae urinary antigen assay in intensive care patients hospitalized for pneumonia. Intensive Care Med 2006;32:1766-72.

223. Smith MD, Derrington $P$, Evans $R$, et al. Rapid diagnosis of bacteremic pneumococcal infections in adults by using the Binax NOW Streptococcus pneumoniae urinary antigen test: a prospective, controlled clinical evaluation. J Clin Microbiol 2003;41:2810-3.

224. Porcel JM, Ruiz-Gonzalez A, Falguera M, et al. Contribution of a pleural antigen assay (Binax NOW) to the diagnosis of pneumococcal pneumonia. Chest 2007; 131:1442-7

225. Sheppard CL, Harrison TG, Kearns AM, et al. Diagnosis of invasive pneumococcal infection by PCR amplification of Streptococcus pneumoniae genomic fragments in blood: a multi-centre comparative study. Commun Dis Public Health 2003;6:221-7.

226. Murdoch DR, Anderson TP, Beynon KA, et al. Evaluation of a PCR assay for detection of Streptococcus pneumoniae in respiratory and nonrespiratory samples from adults with community-acquired pneumonia. J Clin Microbiol 2003;41:63-6.

227. Birtles RJ, Harrison TG, Samuel D, et al. Evaluation of urinary antigen ELISA for diagnosing Legionella pneumophila serogroup 1 infection. J Clin Pathol 1990;43:685-90.

228. Lettinga KD, Verbon A, Weverling GJ, et al. Legionnaires' disease at a Dutch flower show: prognostic factors and impact of therapy. Emerg Infect Dis 2002;8:1448-54.

229. Helbig JH, Uldum So A, Bernander S, et al. Clinical utility of urinary antigen detection for diagnosis of community-acquired, travel-associated, and nosocomial Legionnaires' disease. J Clin Microbiol 2003;41:838-40.

230. Boswell TC, Marshall LE, Kudesia G. False-positive legionella titres in routine clinical serology testing detected by absorption with campylobacter: implications for the serological diagnosis of legionnaires' disease. J Infect 1996;32:23-6.

231. Medical Devices Agency. Mycoplasma pneumoniae antibody assays. Medical Devices Agency Evaluation Report MDA/98/62, 1998.

232. Beersma MF, Dirven K, van Dam AP, et al. Evaluation of 12 commercial tests and the complement fixation test for Mycoplasma pneumoniae-specific immunoglobulin G (lgG) and IgM antibodies, with PCR used as the "gold standard". J Clin Microbiol 2005;43:2277-85.

233. Daxboeck F, Khanakah G, Bauer C, et al. Detection of Mycoplasma pneumoniae in serum specimens from patients with mycoplasma pneumonia by PCR [correction appears in Int J Med Microbiol 2006;296:55]. Int J Med Microbiol 2005;295:279-85

234. Sillis M, White P, Caul EO, et al. The differentiation of Chlamydia species by antigen detection in sputum specimens from patients with community-acquired acute respiratory infections. J Infect 1992;25(Suppl 1):77-86.

235. Garnett $\mathbf{P}$, Brogan 0, Lafong $\mathbf{C}$, et al. Comparison of throat swabs with sputum specimens for the detection of Chlamydia pneumoniae antigen by direct immunofluorescence. J Clin Pathol 1998;51:309-11.

236. Boman J, Allard A, Persson K, et al. Rapid diagnosis of respiratory Chlamydia pneumoniae infection by nested touchdown polymerase chain reaction compared with culture and antigen detection by EIA. J Infect Dis 1997;175:1523-6.

237. Sillis M, Wreghitt TG, Paul ID, et al. Chlamydial respiratory infections. Don't get bogged down by differentiating species. BMJ 1993;307:62-3.

238. Kutlin A, Tsumura N, Emre U, et al. Evaluation of Chlamydia immunoglobulin M $(\operatorname{lgM}), \operatorname{lgG}$, and $\lg A$ rELISAs Medac for diagnosis of Chlamydia pneumoniae infection. Clin Diagn Lab Immunol 1997:4:213-6.

239. Ewig S, Ruiz M, Mensa J, et al. Severe community-acquired pneumonia. Assessment of severity criteria. Am J Respir Crit Care Med 1998;158:1102-8.

240. Meyer RJ, Town Gl, Harrè E, et al. An audit of the assessment and management of adults admitted to Christchurch Hospital with community acquired pneumonia. NZ Med J 1997;110:349-52.

241. Neill AM, Martin IR, Weir R, et al. Community acquired pneumonia: aetiology and usefulness of severity criteria on admission. Thorax 1996;51:1010-6.

242. Farr BM, Sloman AJ, Fisch MJ. Predicting death in patients hospitalized for community-acquired pneumonia. Ann Intern Med 1991;115:428-36.

243. Fine MJ, Smith MA, Carson CA, et al. Prognosis and outcomes of patients with community-acquired pneumonia. A meta-analysis. JAMA 1996;275:134-41.

244. Fine MJ, Singer DE, Hanusa BH, et al. Validation of a pneumonia prognostic index using the MedisGroups Comparative Hospital Database. Am J Med 1993;94:153-9.

245. Hedlund $\mathbf{J}$, Kalin M, Ortqvist A. Recurrence of pneumonia in middle-aged and elderly adults after hospital-treated pneumonia: aetiology and predisposing conditions. Scand J Infect Dis 1997;29:387-92.

246. Conte HA, Chen YT, Mehal W, et al. A prognostic rule for elderly patients admitted with community-acquired pneumonia. Am J Med 1999;106:20-8.

247. Kothe H, Bauer T, Marre R, et al. Outcome of community-acquired pneumonia: influence of age, residence status and antimicrobial treatment. Eur Respir $J$ 2008;32:139-46.

248. Fine MJ, Hanusa BH, Lave JR, et al. Comparison of a disease-specific and a generic severity of illness measure for patients with community-acquired pneumonia. J Gen Intern Med 1995;10:359-68.

249. Pachon J, Prados MD, Capote F, et al. Severe community-acquired pneumonia. Etiology, prognosis, and treatment. Am Rev Respir Dis 1990;142:369-73.

250. Leroy 0, Santré C, Beuscart C, et al. A five-year study of severe communityacquired pneumonia with emphasis on prognosis in patients admitted to an intensive care unit. Intensive Care Med 1995:21:24-31.
251. Fine MJ, Auble TE, Yealy DM, et al. A prediction rule to identify low-risk patients with community-acquired pneumonia. N Engl J Med 1997;336:243-50.

252. Ortqvist A, Hedlund J, Grillner L, et al. Aetiology, outcome and prognostic factors in community-acquired pneumonia requiring hospitalization. Eur Respir $J$ 1990;3:1105-13

253. Garcia-Ordonez MA, Garcia-Jimenez JM, Paez F, et al. Clinical aspects and prognostic factors in elderly patients hospitalised for community-acquired pneumonia. Eur J Clin Microbiol Infect Dis 2001;20:14-9.

254. van Eeden SF, Coetzee AR, Joubert JR. Community-acquired pneumonia-factors influencing intensive care admission. S Afr Med J 1988;73:77-81.

255. Lim WS, van der Eerden MM, Laing $R$, et al. Defining community acquired pneumonia severity on presentation to hospital: an international derivation and validation study. Thorax 2003;58:377-82

256. Gómez J, Baños V, Ruiz Gómez J, et al. Prospective study of epidemiology and prognostic factors in community-acquired pneumonia. Eur J Clin Microbiol Infect Dis 1996;15:556-60.

257. Chalmers JD, Singanayagam A, Hill AT. C-reactive protein is an independent predictor of severity in community-acquired pneumonia. Am J Med 2008;121:219-25.

258. Leroy 0, Georges $\mathrm{H}$, Beuscart $\mathrm{C}$, et al. Severe community-acquired pneumonia in ICUs: prospective validation of a prognostic score. Intensive Care Med 1996;22:1307-14.

259. Hasley PB, Albaum MN, Li YH, et al. Do pulmonary radiographic findings at presentation predict mortality in patients with community-acquired pneumonia? Arch Intern Med 1996;156:2206-12.

260. Feldman C, Kallenbach JM, Levy $\mathrm{H}$, et al. Community-acquired pneumonia of diverse aetiology: prognostic features in patients admitted to an intensive care unit and a "severity of illness" core. Intensive Care Med 1989;15:302-7.

261. Hedlund J, Hansson LO. Procalcitonin and C-reactive protein levels in communityacquired pneumonia: correlation with etiology and prognosis. Infection 2000;28:68-73.

262. Masia M, Gutierrez F, Shum C, et al. Usefulness of procalcitonin levels in community-acquired pneumonia according to the patients' outcome research team pneumonia severity index. Chest 2005;128:2223-9.

263. Boussekey N, Leroy 0, Alfandari S, et al. Procalcitonin kinetics in the prognosis of severe community-acquired pneumonia. Intensive Care Med 2006;32:469-72.

264. Kruger S, Ewig S, Marre R, et al. Procalcitonin predicts patients at low risk of death from community-acquired pneumonia across all CRB-65 classes. Eur Respir $J$ 2008;31:349-55

265. Boussekey N, Leroy 0 , Georges $\mathrm{H}$, et al. Diagnostic and prognostic values of admission procalcitonin levels in community-acquired pneumonia in an intensive care unit. Infection 2005;33:257-63.

266. Tejera A, Santolaria F, Diez ML, et al. Prognosis of community acquired pneumonia (CAP): value of triggering receptor expressed on myeloid cells-1 (TREM-1) and other mediators of the inflammatory response. Cytokine 2007;38:117-23.

267. Aalto H, Takala A, Kautiainen $\mathbf{H}$, et al. Monocyte CD14 and soluble CD14 in predicting mortality of patients with severe community acquired infection. Scand J Infect Dis 2007;39:596-603.

268. Christ-Crain M, Morgenthaler NG, Stolz D, et al. Pro-adrenomedullin to predict severity and outcome in community-acquired pneumonia. Crit Care 2006:10:R96.

269. Coelho L, Povoa P, Almeida E, et al. Usefulness of C-reactive protein in monitoring the severe community-acquired pneumonia clinical course. Crit Care 2007;11(28).

270. Kruger S, Papassotiriou J, Marre R, et al. Pro-atrial natriuretic peptide and provasopressin to predict severity and prognosis in community-acquired pneumonia: results from the German competence network CAPNETZ. Intensive Care Med 2007:33:2069-78.

271. Prat C, Lacoma A, Dominguez J, et al. Midregional pro-atrial natriuretic peptide as a prognostic marker in pneumonia. J Infect 2007;55:400-7.

272. Brunkhorst FM, Al-Nawas B, Krummenauer F, et al. Procalcitonin, C-reactive protein and APACHE II score for risk evaluation in patients with severe pneumonia Clin Microbiol Infect 2002;8:93-100.

273. Shilon Y, Shitrit AB-G, Rudensky B, et al. A rapid quantitative D-dimer assay at admission correlates with the severity of community acquired pneumonia. Blood Coagul Fibrinolysis 2003;14:745-8.

274. Querol-Ribelles JM, Tenias JM, Grau E, et al. Plasma D-dimer levels correlate with outcomes in patients with community-acquired pneumonia. Chest 2004; 126:1087-92.

275. Tapson VF. Risk factors in community-acquired pneumonia: can they be quantified? Eur Respir Rev 1998:8:291-4.

276. Niederman MS, Feldman C, Richards GA. Combining information from prognostic scoring tools for CAP: an American view on how to get the best of all worlds [comment]. Eur Respir J 2006;27:9-11.

277. Gleason PP, Kapoor WN, Stone RA, et al. Medical outcomes and antimicrobial costs with the use of the American Thoracic Society guidelines for outpatients with community-acquired pneumonia. JAMA 1997;278:32-9.

278. Marras TK, Chan CK. Use of guidelines in treating community-acquired pneumonia Chest 1998:113:1689-94.

279. Atlas SJ, Benzer TI, Borowsky LH, et al. Safely increasing the proportion of patients with community-acquired pneumonia treated as outpatients: an interventional trial. Arch Intern Med 1998;158:1350-6.

280. Yealy DM, Auble TE, Stone RA, et al. Effect of increasing the intensity of implementing pneumonia guidelines: a randomized, controlled trial. Ann Intern Med 2005;143:881-94. 
281. Renaud B, Coma E, Labarere J, et al. Routine use of the Pneumonia Severity Index for guiding the site-of-treatment decision of patients with pneumonia in the emergency department: a multicenter, prospective, observational, controlled cohort study. Clin Infect Dis 2007:44:41-9.

282. Richeldi L, De Guglielmo M, Fabbri LM, et al. Management of CAP using a validated risk score. Thorax 2005;60:172-3

283. Barlow G, Nathwani D, Williams F, et al. Reducing door-to-antibiotic time in community-acquired pneumonia: controlled before-and-after evaluation and costeffectiveness analysis. Thorax 2007;62:67-74.

284. Tang CM, Macfarlane JT. Early management of younger adults dying of community acquired pneumonia. Respir Med 1993;87:289-94.

285. Gladman J, Barer D, Venkatesan P, et al. Outcome of pneumonia in the elderly: a hospital survey. Clin Rehab 1991;5:201-5.

286. Capelastegui A, Espana PP, Quintana JM, et al. Validation of a predictive rule for the management of community-acquired pneumonia. Eur Respir J 2006:27:151-7.

287. Myint PK, Kamath AV, Vowler SL, et al. Severity assessment criteria recommended by the British Thoracic Society (BTS) for community-acquired pneumonia (CAP) and older patients. Should SOAR (systolic blood pressure, oxygenation, age and respiratory rate) criteria be used in older people? A compilation study of two prospective cohorts. Age Ageing 2006;35:286-91.

288. Aujesky D, Auble TE, Yealy DM, et al. Prospective comparison of three validated prediction rules for prognosis in community-acquired pneumonia. Am J Med 2005; 118:384-92.

289. Barlow G, Nathwani D, Davey P. The CURB65 pneumonia severity score outperforms generic sepsis and early warning scores in predicting mortality in community-acquired pneumonia. Thorax 2007:62:253-9.

290. Spindler C, Ortqvist A. Prognostic score systems and community-acquired bacteraemic pneumococcal pneumonia. Eur Respir J 2006;28:816-23.

291. Buising KL, Thursky KA, Black JF, et al. A prospective comparison of severity scores for identifying patients with severe community acquired pneumonia: reconsidering what is meant by severe pneumonia. Thorax 2006:61:419-24.

292. Shindo Y, Sato S, Maruyama E, et al. Comparison of severity scoring systems A-DROP and CURB-65 for community-acquired pneumonia. Respirology 2008; 13:731-5

293. Loh L-C. Community-acquired pneumonia in Malaysian patients: addition of macrolide and the use of BTS "curb" index to assess severity. Med J Malaysia 2006;61:128-30.

294. Espana PP, Capelastegui A, Gorordo I, et al. Development and validation of a clinical prediction rule for severe community-acquired pneumonia. Am J Respir Crit Care Med 2006;174:1249-56.

295. Ananda-Rajah MR, Charles PG, Melvani S, et al. Comparing the pneumonia severity index with CURB-65 in patients admitted with community acquired pneumonia. Scand J Infect Dis 2008:40:293-300.

296. Bauer TT, Ewig S, Marre R, et al. CRB-65 predicts death from community-acquired pneumonia. J Intern Med 2006;260:93-101.

297. Schaaf B, Kruse J, Rupp J, et al. Sepsis severity predicts outcome in communityacquired pneumococcal pneumonia. Eur Respir J 2007;30:517-24.

298. Man SY, Lee N, Ip M, et al. Prospective comparison of three predictive rules for assessing severity of community-acquired pneumonia in Hong Kong. Thorax 2007;62:348-53.

299. Bont J, Hak E, Hoes AW, et al. A prediction rule for elderly primary-care patients with lower respiratory tract infections. Eur Respir J 2007;29:969-75.

300. Myint PK, Kamath AV, Vowler SL, et al. Simple modification of CURB-65 better identifies patients including the elderly with severe CAP. Thorax 2007;62:1015-6.

301. Ewig S, de Roux A, Bauer T, et al. Validation of predictive rules and indices of severity for community acquired pneumonia. Thorax 2004:59:421-7.

302. Buising KL, Thursky KA, Black JF, et al. Identifying severe community-acquired pneumonia in the emergency department: a simple clinical prediction tool. Emerg Med Australas 2007;19:418-426.

303. Charles PG, Wolfe R, Whitby M, et al. SMART-COP: a tool for predicting the need for intensive respiratory or vasopressor support in community-acquired pneumonia. Clin Infect Dis 2008; 47:375-84.

304. Leroy 0, Devos P, Guery B, et al. Simplified prediction rule for prognosis of patients with severe community-acquired pneumonia in ICUs. Chest 1999;116:157-65.

305. Farr BM, Woodhead MA, Macfarlane JT, et al. Risk factors for community-acquired pneumonia diagnosed by general practitioners in the community. Respir Med 2000;94:422-7.

306. Tydeman DE, Chandler AR, Graveling BM, et al. An investigation into the effects of exercise tolerance training on patients with chronic airways obstruction. Physiotherapy 1984;70:261-4

307. LaCroix AZ, Lipson S, Miles TP, et al. Prospective study of pneumonia hospitalizations and mortality of U.S. older people: the role of chronic conditions, health behaviors, and nutritional status. Public Health Rep 1989;104:350-60.

308. Levin KP, Hanusa BH, Rotondi A, et al. Arterial blood gas and pulse oximetry in initial management of patients with community-acquired pneumonia. J Gen Intern Med 2001;16:590-8

309. Fulmer JD, Snider GL. American College of Chest Physicians/National Heart, Lung, and Blood Institute National Conference on Oxygen Therapy. Heart Lung 1984;13:550-62.

310. Marrie TJ, Lau CY, Wheeler SL, et al. A controlled trial of a critical pathway for treatment of community-acquired pneumonia. CAPITAL Study Investigators. Community-Acquired Pneumonia Intervention Trial Assessing Levofloxacin. JAMA 2000:283:749-55
311. Scottish Intercollegiate Guideline Network. Prophylaxis of venous thromboembolism. 2002. http://www.sign.ac.uk/guidelines/fulltext/62/index.html.

312. Mismetti P, Laporte-Simitsidis S, Tardy B, et al. Prevention of venous thromboembolism in internal medicine with unfractionated or low-molecular-weight heparins: a meta-analysis of randomised clinical trials. Thromb Haemost 2000;83:14-9.

313. NHS Lothian. NHS Lothian Antithrombotic Guide. 2006

314. Mundy LM, Leet TL, Darst K, et al. Early mobilization of patients hospitalized with community-acquired pneumonia. Chest 2003;124:883-9.

315. Tydeman DE. An investigation into the effectiveness of physiotherapy in the treatment of patients with community acquired pneumonia. Physio Pract 1989;5:75-8.

316. Björkqvist M, Wiberg B, Bodin L, et al. Bottle-blowing in hospital-treated patients with community-acquired pneumonia. Scand J Infect Dis 1997:29:77-82.

317. National Institute for Health and Clinical Excellence (NICE). Acutely ill patients in hospital. Clinical Guideline 50. July 2007

318. Menendez R, Cavalcanti M, Reyes S, et al. Markers of treatment failure in hospitalised community acquired pneumonia. Thorax 2008;63:447-52.

319. Halm EA, Fine MJ, Kapoor WN, et al. Instability on hospital discharge and the risk of adverse outcomes in patients with pneumonia. Arch Intern Med 2002;162:1278-84.

320. Dagan E, Novack V, Porath A. Adverse outcomes in patients with community acquired pneumonia discharged with clinical instability from Internal Medicine Department. Scand J Infect Dis 2006;38:860-6.

321. Bone RC, Balk RA, Cerra FB, et al. Definitions for sepsis and organ failure and guidelines for the use of innovative therapies in sepsis. The ACCP/SCCM Consensus Conference Committee. American College of Chest Physicians/Society of Critical Care Medicine. Chest 1992;101:1644-55.

322. Demoule A, Girou E, Richard J-C, et al. Benefits and risks of success or failure of noninvasive ventilation. Intensive Care Med 2006;32:1756-65.

323. Sinuff T, Cook DJ. Health technology assessment in the ICU: noninvasive positive pressure ventilation for acute respiratory failure. J Crit Care 2003:18:59-67.

324. Keenan SP, Sinuff T, Cook DJ, et al. Does noninvasive positive pressure ventilation improve outcome in acute hypoxemic respiratory failure? A systematic review. Crit Care Med 2004;32:2516-23.

325. Confalonieri M, Potena A, Carbone G, et al. Acute respiratory failure in patients with severe community-acquired pneumonia. A prospective randomized evaluation of noninvasive ventilation. Am J Respir Crit Care Med 1999:160:1585-91.

326. Delclaux C, L'Her E, Alberti C, et al. Treatment of acute hypoxemic nonhypercapnic respiratory insufficiency with continuous positive airway pressure delivered by a face mask: a randomized controlled trial. JAMA 2000:284:2352-60.

327. Bernard GR, Vincent JL, Laterre PF, et al. Efficacy and safety of recombinant human activated protein C for severe sepsis. N Engl J Med 2001;344:699-709.

328. Laterre P-F, Garber G, Levy H, et al. Severe community-acquired pneumonia as a cause of severe sepsis: data from the PROWESS study. Crit Care Med 2005:33:952-61.

329. Marti-Carvajal A, Salanti G, Cardona AF. Human recombinant activated protein C for severe sepsis. Cochrane Database Syst Rev 2008;(1):CD004388.

330. Confalonieri M, Urbino R, Potena A, et al. Hydrocortisone infusion for severe community-acquired pneumonia: a preliminary randomized study. Am J Respir Crit Care Med 2005:171:242-8.

331. Gorman SK, Slavik RS, Marin J. Corticosteroid treatment of severe communityacquired pneumonia. Ann Pharmacother 2007;41:1233-7.

332. Sprung CL, Annane D, Keh D, et al. Hydrocortisone therapy for patients with septic shock. N Engl J Med 2008;358:111-24.

333. Cheng AC, Stephens DP, Currie BJ. Granulocyte-colony stimulating factor (G-CSF) as an adjunct to antibiotics in the treatment of pneumonia in adults. Cochrane Database Syst Rev 2007;(2):CD004400.

334. Leroy 0, Guilley J, Georges $\mathrm{H}$, et al. Effect of hospital-acquired ventilator-associated pneumonia on mortality of severe community-acquired pneumonia. J Crit Care 1999;14:12-9.

335. Caine M, Lim WS, Macfarlane JT, et al. Keeping patients informed about their illness: community acquired pneumonia. Thorax 2000:55(Suppl 3):A71.

336. Impallomeni M, Galletly NP, Wort SJ, et al. Increased risk of diarrhoea caused by Clostridium difficile in elderly patients receiving cefotaxime. BMJ 1995;311:1345-6

337. Riley TV. Clostridium difficile: a pathogen of the nineties. Eur J Clin Microbiol Infect Dis 1998;17:137-41.

338. Zadik PM, Moore AP. Antimicrobial associations of an outbreak of diarrhoea due to Clostridium difficile. J Hosp Infect 1998;39:189-93.

339. MacGowan AP, Feeney R, Brown I, et al. Health care resource utilization and antimicrobial use in elderly patients with community-acquired lower respiratory tract infection who develop Clostridium difficile-associated diarrhoea. J Antimicrob Chemother 1997;39:537-41.

340. Settle CD, Wilcox MH, Fawley WN, et al. Prospective study of the risk of Clostridium difficile diarrhoea in elderly patients following treatment with cefotaxime or piperacillin-tazobactam. Aliment Pharmacol Ther 1998;12:1217-23.

341. Spencer RC. The role of antimicrobial agents in the aetiology of Clostridium difficileassociated disease. J Antimicrob Chemother 1998;41(Suppl C):21-7.

342. Ludlum H, Brown N, Sule 0, et al. An antibiotic policy associated with reduced risk of Clostridium difficile-associated diarrhoea. Age Aging 1999;28:578-80.

343. Johnson AP. Antibiotic resistance among clinically important gram-positive bacteria in the UK. J Hosp Infect 1998:40:17-26. 
344. Felmingham D, Washington J. Trends in the antimicrobial susceptibility of bacterial respiratory tract pathogens_-findings of the Alexander Project 1992-1996. $J$ Chemother 1999;11(Suppl 1):5-21.

345. Pallares $\mathbf{R}$, Linares $\mathrm{J}$, Vadillo $\mathrm{M}$, et al. Resistance to penicillin and cephalosporin and mortality from severe pneumococcal pneumonia in Barcelona, Spain. N Engl J Med 1995; 333:474-80.

346. Einarsson S, Kristjansson M, Kristinsson KG, et al. Pneumonia caused by penicillinnon-susceptible and penicillin-susceptible pneumococci in adults: a case-control study. Scand J Infect Dis 1998;30:253-6.

347. Klugman K, Feldman C. The clinical relevance of antibiotic resistance in the management of pneumoccocal pneumonia. Infect Dis Clin Pract 1998;7:180-4.

348. Pallares R, Viladrich PF, Linares J, et al. Impact of antibiotic resistance on chemotherapy for pneumococcal infections. Microb Drug Resist 1998;4:339-47.

349. Garau J, Aguilar L, Rodriguez-Creixems M, et al. Influence of comorbidity and severity on the clinical outcome of bacteremic pneumococcal pneumonia treated with beta-lactam monotherapy. J Chemother 1999;11:266-72.

350. Ewig S, Kleinfeld T, Bauer T, et al. Comparative validation of prognostic rules for community-acquired pneumonia in an elderly population. Eur Respir $J$ 1999;14:370-5.

351. Farrell DJ, Felmingham D, Shackcloth J, et al. Non-susceptibility trends and serotype distributions among Streptococcus pneumoniae from community-acquired respiratory tract infections and from bacteraemias in the UK and Ireland, 1999 to 2007. J Antimicrob Chemother 2008:62 (Suppl 2):ii87-95.

352. Chen DK, McGeer A, de Azavedo JC, et al. Decreased susceptibility of Streptococcus pneumoniae to fluoroquinolones in Canada. Canadian Bacterial Surveillance Network. N Engl J Med 1999;341:233-9.

353. Powell M, Yeo SF, Seymour A, et al. Antimicrobial resistance in Haemophilus influenzae from England and Scotland in 1991. J Antimicrob Chemother 1992;29:547-54.

354. Morrissey I, Maher K, Williams L, et al. Non-susceptibility trends among Haemophilus influenzae and Moraxella catarrhalis from community-acquired respiratory tract infections in the UK and Ireland, 1999-2007. J Antimicrob Chemother 2008;62(Suppl 2):ii97-103.

355. Andrews $\mathbf{J}$, Ashby J, Jevons $\mathrm{G}$, et al. A comparison of antimicrobial resistance rates in Gram-positive pathogens isolated in the UK from October 1996 to January 1997 and October 1997 to January 1998. J Antimicrob Chemother 2000:45:285-93.

356. Nielsen K, Bangsborg JM, Hoiby N. Susceptibility of Legionella species to five antibiotics and development of resistance by exposure to erythromycin, ciprofloxacin, and rifampicin. Diagn Microbiol Infect Dis 2000;36:43-8.

357. Lode H, Garau J, Grassi C, et al. Treatment of community-acquired pneumonia: a randomized comparison of sparfloxacin, amoxycillin-clavulanic acid and erythromycin. Eur Respir J 1995;8:1999-2007.

358. Soman A, Honeybourne D, Andrews $\mathrm{J}$, et al. Concentrations of moxifloxacin in serum and pulmonary compartments following a single $400 \mathrm{mg}$ oral dose in patients undergoing fibre-optic bronchoscopy. J Antimicrob Chemother 1999;44:835-8.

359. Hoffken G, Meyer HP, Winter J, et al. The efficacy and safety of two oral moxifloxacin regimens compared to oral clarithromycin in the treatment of community-acquired pneumonia. Respir Med 2001;95:553-64.

360. Petitpretz P, Arvis P, Marel M, et al. Oral moxifloxacin vs high-dosage amoxicillin in the treatment of mild-to-moderate, community-acquired, suspected pneumococcal pneumonia in adults. Chest 2001;119:185-95.

361. Fogarty C, Grossman C, Williams J, et al. Efficacy and safety of moxifloxacin vs. clarithromycin for community-acquired pneumonia. Infect Med 1999:748-63.

362. Torres A, Muir JF, Corris P, et al. Effectiveness of oral moxifloxacin in standard firstline therapy in community-acquired pneumonia. Eur Respir J 2003;21:135-43.

363. Anon. Britsh National Formulary. London: BMJ Publishing Group and RPS Publishing, 2008.

364. Anderson G, Esmonde TS, Coles S, et al. A comparative safety and efficacy study of clarithromycin and erythromycin stearate in community-acquired pneumonia. $J$ Antimicrob Chemother 1991;27(Suppl A):117-24.

365. Chien SM, Pichotta P, Siepman N, et al. Treatment of community-acquired pneumonia. A multicenter, double-blind, randomized study comparing clarithromycin with erythromycin. Canada-Sweden Clarithromycin-Pneumonia Study Group. Chest 1993:103:697-701.

366. Green JA, Butler T, Todd WM. Randomized double-blind trial of the comparative efficacy and safety of cefpodoxime proxetil and cefaclor in the treatment of acute community-acquired pneumonia. Curr Ther Res 1994;55:1003-15.

367. Rizzato G, Allegra L. Efficacy and tolerability of a teicoplanin-ciprofloxacin combination in severe community-acquired pneumonia. Clin Drug Invest 1997; 14:337-45.

368. Bohte R, van't Wout JW, Lobatto $S$, et al. Efficacy and safety of azithromycin versus benzylpenicillin or erythromycin in community-acquired pneumonia. Eur J Clin Microbiol Infect Dis 1995;14:182-7.

369. Chan R, Hemeryck L, O'Regan M, et al. Oral versus intravenous antibiotics for community acquired lower respiratory tract infection in a general hospital: open, randomised controlled trial. BMJ 1995;310:1360-2.

370. Plouffe JF, Herbert MT, File TM Jr, et al. Ofloxacin versus standard therapy in treatment of community-acquired pneumonia requiring hospitalization. Pneumonia Study Group. Antimicrob Agents Chemother 1996;40:1175-9.

371. File TM Jr, Segreti J, Dunbar L, et al. A multicenter, randomized study comparing the efficacy and safety of intravenous and/or oral levofloxacin versus ceftriaxone and/or cefuroxime axetil in treatment of adults with community-acquired pneumonia. Antimicrob Agents Chemother 1997:41:1965-72.
372. Van den Brande $\mathbf{P}$, Vondra V, Vogel $F$, et al. Sequential therapy with cefuroxime followed by cefuroxime axetil in community-acquired pneumonia. Chest 1997:112:406-15.

373. Vetter N, Stamler D, O'Neill S, et al. Clarithromycin vs combined cefuroxime and erythromycin in the treatment of hospitalized community-acquired pneumonia patients: intravenous followed by oral therapy. Clin Drug Invest 1997;14:439-49.

374. O'Doherty B, Muller 0 . Randomized, multicentre study of the efficacy and tolerance of azithromycin versus clarithromycin in the treatment of adults with mild to moderate community-acquired pneumonia. Azithromycin Study Group. Eur J Clin Microbiol Infect Dis 1998;17:828-33.

375. Carbon C, Ariza H, Rabie WJ, et al. Comparative study of levofloxacin and amoxycillin/clavulanic acid in adults with mild-to-moderate community-acquired pneumonia. Clin Microbiol Infect 1999;5:724-32.

376. Rovira E, Martinez-Moragon E, Belda A, et al. Treatment of community-acquired pneumonia in outpatients: randomized study of clarithromycin alone versus clarithromycin and cefuroxime. Respiration 1999;66:413-8.

377. Johnson RH, Levine S, Traub SL. Sequential intravenous/oral ciprofloxacin compared with parenteral ceftrixone in the treatment of hospitalized patients with community-acquired pneumonia. Infect Dis Clin Pract 1996:5:265-72.

378. Bartoloni A, Strohmeyer M, Corti G, et al. Multicenter randomized trial comparing meropenem (1.5 g daily) and imipenem/cilastatin (2 $\mathrm{g}$ daily) in the hospital treatment of community-acquired pneumonia. Drugs Exp Clin Res 1999;25:243-52.

379. Garau J, Twynholm M, Garcia-Mendez E, et al. Oral pharmacokinetically enhanced co-amoxiclav 2000/125 mg, twice daily, compared with co-amoxiclav 875/125 mg, three times daily, in the treatment of community-acquired pneumonia in European adults. J Antimicrob Chemother 2003;52:826-36.

380. Siquier B, Sanchez-Alvarez J, Garcia-Mendez E, et al. Efficacy and safety of twicedaily pharmacokinetically enhanced amoxicillin/clavulanate (2000/125 mg) in the treatment of adults with community-acquired pneumonia in a country with a high prevalence of penicillin-resistant Streptococcus pneumoniae. J Antimicrob Chemother 2006; $57: 536-45$.

381. Dunbar LM. Current issues in the management of bacterial respiratory tract disease: the challenge of antibacterial resistance. Am J Med Sci 2003;326:360-8.

382. Baumgartner JD, Glauser MP. Tolerance study of ceftriaxone compared with amoxicillin in patients with pneumonia. Am J Med 1984;77:54-8.

383. Fredlund $\mathbf{H}$, Bodin L, Back E, et al. Antibiotic therapy in pneumonia: a comparative study of parenteral and oral administration of penicillin. Scand J Infect Dis 1987:19:459-66.

384. Zuck P, Rio Y, Ichou F. Efficacy and tolerance of cefpodoxime proxetil compared with ceftriaxone in vulnerable patients with bronchopneumonia. J Antimicrob Chemother 1990;26(Suppl E):71-7.

385. Poirier R. Comparative study of clarithromycin and roxithromycin in the treatment of community-acquired pneumonia. J Antimicrob Chemother 1991;27(Suppl A):109-16.

386. Koulla-Shiro S, Kuaban C, Auckenthaler R, et al. Adult response to initial treatment with ampicillin in community acquired pneumonia in Yaounde, Cameroon. Cent Afr J Med 1993:39:188-92.

387. Gaillat J, Bru JP, Sedallian A. Penicillin G/ofloxacin versus erythromycin/amoxicillinclavulanate in the treatment of severe community-acquired pneumonia. Eur $\mathrm{J}$ Clin Microbiol Infect Dis 1994;13:639-44.

388. Low DE, Mandell LA. A prospective open-label multi-centre trial on the use of $1 \mathrm{~g}$, once daily ceftriaxone in lower respiratory tract infections. Can $\mathrm{J}$ Infect Dis 1994;5(Suppl C):3-8C.

389. Uzun 0, Hayran M, Akova M, et al. Efficacy of a three-day course of azithromycin in the treatment of community-acquired pneumococcal pneumonia. Preliminary report. $J$ Chemother 1994;6:53-7.

390. Jang TN, Liu CY, Wang FD, et al. A randomized comparative study on the safety and efficacy of clarithromycin and erythromycin in treating community-acquired pneumonia. Zhonghua Yi Xue Za Zhi (Taipei) 1995;55:302-6.

391. Rizzato G, Montemurro L, Fraioli P, et al. Efficacy of a three day course of azithromycin in moderately severe community-acquired pneumonia. Eur Respir $J$ 1995:8:398-402.

392. Oh HM, Ng AW, Lee SK. Cefuroxime compared to amoxicillin-clavulanic acid in the treatment of community-acquired pneumonia. Singapore Med J 1996;37:255-7.

393. Siegel RE, Halpern NA, Almenoff PL, et al. A prospective randomized study of inpatient IV antibiotics for community-acquired pneumonia. The optimal duration of therapy. Chest 1996;110:965-71.

394. Genne D, Siegrist HH, Humair L, et al. Clarithromycin versus amoxicillin-clavulanic acid in the treatment of community-acquired pneumonia. Eur J Clin Microbiol Infect Dis 1997; 16:783-8.

395. Ho A, Leung R, Lai CK, et al. Hospitalized patients with community-acquired pneumonia in Hong Kong: a randomized study comparing imipenem/cilastatin and ceftazidime. Respiration 1997;64:224-8.

396. Cabellos C, Ariza J, Barreiro B, et al. Current usefulness of procaine penicillin in the treatment of pneumococcal pneumonia. Eur J Clin Microbiol Infect Dis 1998:17:265-8.

397. Fogarty CM, Sullivan JG, Chattman MS, et al. Once a day levofloxacin in the treatment of mild to moderate and severe community-acquired pneumonia in adults. Infect Dis Clin Pract 1998:7:400-7.

398. Rhew DC, Hackner D, Henderson L, et al. The clinical benefit of in-hospital observation in 'low-risk' pneumonia patients after conversion from parenteral to oral antimicrobial therapy. Chest 1998:113:142-6. 
399. Speich R, Imhof E, Vogt M, et al. Efficacy, safety, and tolerance of piperacillin/ tazobactam compared to co-amoxiclav plus an aminoglycoside in the treatment of severe pneumonia. Eur J Clin Microbiol Infect Dis 1998;17:313-7.

400. Shorr AF, Khashab MM, Xiang JX, et al. Levofloxacin 750-mg for 5 days for the treatment of hospitalized Fine risk class III/IV community-acquired pneumonia patients. Respir Med 2006;100:2129-36.

401. File TM Jr. Community-associated methicillin-resistant Staphylococcus aureas: not only a cause of skin infections, also a new cause of pneumonia. Curr Opin Infect Dis 2005;18:123-4.

402. Fogarty C, Siami G, Kohler R, et al. Multicenter, open-label, randomized study to compare the safety and efficacy of levofloxacin versus ceftriaxone sodium and erythromycin followed by clarithromycin and amoxicillin-clavulanate in the treatment of serious community-acquired pneumonia in adults. Clin Infect Dis 2004;38(Suppl 1):S16-23.

403. Plouffe JF, Breiman RF, Fields BS, et al. Azithromycin in the treatment of Legionella pneumonia requiring hospitalization. Clin Infect Dis 2003;37:1475-80.

404. Welte T, Petermann W, Schurmann D, et al. Treatment with sequential intravenous or oral moxifloxacin was associated with faster clinical improvement than was standard therapy for hospitalized patients with community-acquired pneumonia who received initial parenteral therapy. Clin Infect Dis 2005:41:1697-705.

405. Lode H, Grossman C, Choudhri S, et al. Sequential IV/PO moxifloxacin treatment of patients with severe community-acquired pneumonia. Respir Med 2003;97:1134-42.

406. Wallace RJ Jr, Martin RR, Quinones FJ, et al. Ceforanide and cefazolin therapy of pneumonia: comparative clinical trial. Antimicrob Agents Chemother 1981;20:648-52.

407. Grieco MM, Lange M, Daniels JA, et al. Single-blind controlled study of ceftizoxime and cefamandole in the treatment of community-acquired pneumonia. J Antimicrob Chemother 1982;10(Suppl C):223-5.

408. Wallace RJ Jr, Niefield SL, Waters S, et al. Comparative trial of cefonicid and cefamandole in the therapy of community-acquired pneumonia. Antimicrob Agents Chemother 1982:21:231-5

409. Keeton GR, Kehoe B, Phillips SW, et al. Ceftazidime and cefamandole in the treatment of pneumonia. J Antimicrob Chemother 1983;12(Suppl A):27-30.

410. Geckler RW, McCormack GD, Goodman JS. Comparison of cefonicid and cefamandole for the treatment of community-acquired infections of the lower respiratory tract. Rev Infect Dis 1984;6(Suppl 4):S847-52

411. Weber DJ, Calderwood SB, Karchmer AW, et al. Ampicillin versus cefamandole as initial therapy for community-acquired pneumonia. Antimicrob Agents Chemother 1987:31:876-82.

412. Carbon C, Leophonte P, Petitpretz P, et al. Efficacy and safety of temafloxacin versus those of amoxicillin in hospitalized adults with community-acquired pneumonia. Antimicrob Agents Chemother 1992;36:833-9.

413. Kissling M. Cefetamet pivoxil in community-acquired pneumonia: an overview. Curr Med Res Opin 1992;12:631-9.

414. Wiesner B, Wilen-Rosenqvist G, Lehtonen L. Twice daily dosing of erythromycin acistrate in the treatment of acute bronchitis and pneumonia. Arzneimittelforschung 1993:43:1014-7.

415. Aquino R, Lansang MA, Salvador A, et al. The effectiveness and safety of ceftriaxone to cefetamet pivoxil vs ceftazidime to cefetamet pivoxil vs cefoperazone to cefetamet pivoxil in the treatment of community-acquired pneumonia in adult Filipinos. Phil J Intern Med 1994;31:237-40.

416. Liippo K, Tala E, Puolijoki $\mathrm{H}$, et al. A comparative study of dirithromycin and erythromycin in bacterial pneumonia. J Infect 1994;28:131-9.

417. Williams D, Perri M, Zervos MJ. Randomized comparative trial with ampicillin/ sulbactam versus cefamandole in the therapy of community acquired pneumonia. Eur J Clin Microbiol Infect Dis 1994;13:293-8.

418. De Palma M, Rocchi D, Canepa G, et al. Single daily dose of cefodizime in patients with community-acquired pneumonia: an open-label, controlled, randomized study. Clin Ther 1995;17:413-24

419. Hopkins S, Williams D. Five-day azithromycin in the treatment of patients with community-acquired pneumonia. Curr Ther Res 1995;56:915-25.

420. Aubier M, Lode H, Gialdroni-Grassi G, et al. Sparfloxacin for the treatment of community-acquired pneumonia: a pooled data analysis of two studies. J Antimicrob Chemother 1996;37(Suppl A):73-82.

421. Hernandez JM, Sides GD, Conforti PM, et al. Clinical efficacy of dirithromycin in patients with bacteremic pneumonia. Clin Ther 1996;18:1128-38.

422. Ortquist A, Valtonen $\mathrm{M}$, Cars 0 , et al. Oral empiric treatment of communityacquired pneumonia. A multicenter, double-blind, randomized study comparing sparfloxacin with roxithromycin. The Scandinavian Sparfloxacin Study Group. Chest 1996;110:1499-506.

423. Portier H, May T, Proust A. Comparative efficacy of sparfloxacin in comparison with amoxycillin plus ofloxacin in the treatment of community-acquired pneumonia. French Study Group. J Antimicrob Chemother 1996;37(Suppl A):83-91.

424. Rubinstein E. Safety profile of sparfloxacin in the treatment of respiratory tract infections. J Antimicrob Chemother 1996;37(Suppl A):145-60.

425. Donowitz GR, Brandon ML, Salisbury JP, et al. Sparfloxacin versus cefaclor in the treatment of patients with community-acquired pneumonia: a randomized, doublemasked, comparative, multicenter study. Clin Ther 1997;19:936-53.

426. Drehobl M, Bianchi $\mathrm{P}, \mathrm{Keyserling} \mathrm{CH}$, et al. Comparison of cefdinir and cefaclor in treatment of community-acquired pneumonia. Antimicrob Agents Chemother 1997:41:1579-83.
427. Ragnar NS. Atypical pneumonia in the Nordic countries: aetiology and clinical results of a trial comparing fleroxacin and doxycycline. Nordic Atypical Pneumonia Study Group. J Antimicrob Chemother 1997;39:499-508.

428. O'Doherty B, Dutchman DA, Pettit R, et al. Randomized, double-blind, comparative study of grepafloxacin and amoxycillin in the treatment of patients with communityacquired pneumonia. J Antimicrob Chemother 1997;40(Suppl A):73-81.

429. Aubier M, Verster R, Regamey C, et al. Once-daily sparfloxacin versus high-dosage amoxicillin in the treatment of community-acquired, suspected pneumococcal pneumonia in adults. Sparfloxacin European Study Group. Clin Infect Dis 1998;26:1312-20

430. Salvarezza CR, Mingrone $\mathrm{H}$, Fachinelli $\mathrm{H}$, et al. Comparison of roxithromycin with cefixime in the treatment of adults with community-acquired pneumonia. J Antimicrob Chemother 1998;41(Suppl B):75-80.

431. Tremolieres F, de Kock F, Pluck N, et al. Trovafloxacin versus high-dose amoxicillin $(1 \mathrm{~g}$ three times daily) in the treatment of community-acquired bacterial pneumonia. Eur J Clin Microbiol Infect Dis 1998;17:447-53.

432. Zervos M, Nelson M. Cefepime versus ceftriaxone for empiric treatment of hospitalized patients with community-acquired pneumonia. The Cefepime Study Group. Antimicrob Agents Chemother 1998;42:729-33.

433. Daniel R. Trovafloxacin vs high dose amoxicillin in the treatment of communityacquired bacterial pneumonia. Drugs 1999;58(Suppl 2):304-5.

434. Daniel R. Oral trovafloxacin compared with amoxicillin (plus optional erythromycin) for the treatment of mild to moderate community-acquired pneumonia. Drugs 1999;58(Suppl 2):320-2

435. Grossman RF, Campbell DA, Landis SJ, et al. Treatment of community-acquired pneumonia in the elderly: the role of cefepime, a fourth-generation cephalosporin $J$ Antimicrob Chemother 1999;43:549-54

436. Moola S, Hagberg L, Churchyard GA, et al. A multicenter study of grepafloxacin and clarithromycin in the treatment of patients with community-acquired pneumonia. Chest 1999;116:974-83.

437. Peterson J. Oral trovafloxacin vs oral cefuroxime \pm erythromycin in community acquired pneumonia. Drugs 1999;58(Suppl 2):301-3

438. Ramirez J, Unowsky J, Talbot GH, et al. Sparfloxacin versus clarithromycin in the treatment of community-acquired pneumonia. Clin Ther 1999;21:103-17.

439. Leophonte $\mathbf{P}$, File T, Feldman C. Gemifloxacin once daily for 7 days compared to amoxicillin/clavulanic acid thrice daily for 10 days for the treatment of community-acquired pneumonia of suspected pneumococcal origin. Respir Med 2004;98:708-20.

440. Tellier G, Niederman MS, Nusrat R, et al. Clinical and bacteriological efficacy and safety of 5 and 7 day regimens of telithromycin once daily compared with a 10 day regimen of clarithromycin twice daily in patients with mild to moderate communityacquired pneumonia. J Antimicrob Chemother 2004;54:515-23.

441. Drehobl MA, De Salvo MC, Lewis DE, et al. Single-dose azithromycin microspheres vs clarithromycin extended release for the treatment of mild-to-moderate community-acquired pneumonia in adults. Chest 2005;128:2230-7.

442. Blaser MJ, Klaus BD, Jacobson JA, et al. Comparison of cefadroxil and cephalexin in the treatment of community-acquired pneumonia. Antimicrob Agents Chemother 1983:24:163-7.

443. Cassell GH, Drnec J, Waites KB, et al. Efficacy of clarithromycin against Mycoplasma pneumoniae. J Antimicrob Chemother 1991;27(Suppl A):47-59.

444. Morales J0, Snead H. Efficacy and safety of intravenous cefotaxime for treating pneumonia in outpatients. Am J Med 1994;97:28-33.

445. Ramirez JA. Switch therapy in community-acquired pneumonia. Diagn Microbiol Infect Dis 1995;22:219-23.

446. Higuera $\mathbf{F}$, Hidalgo $\mathrm{H}$, Feris J, et al. Comparison of oral cefuroxime axetil and oral amoxycillin/clavulanate in the treatment of community-acquired pneumonia. $J$ Antimicrob Chemother 1996;37:555-64

447. Berman SJ, Sieger B, Geckler W, et al. A comparative study of meropenem and ceftazidime in the treatment of patients hospitalized with community-acquired pneumonia. Curr Ther Res 1997; 58:903-16.

448. Ailani RK, Agastya G, Mukunda BN, et al. Doxycycline is a cost-effective therapy for hospitalized patients with community-acquired pneumonia. Arch Intern Med 1999;159:266-70.

449. Schonwald S, Kuzman I, Oreskovic K, et al. Azithromycin: single $1.5 \mathrm{~g}$ dose in the treatment of patients with atypical pneumonia syndrome-a randomized study. Infection 1999;27:198-202.

450. Sherer Y, Bakshi E, Rotman P, et al. Comparative clinical study of cefonicid, chloramphenicol, and penicillin in community-acquired pneumonia. Int J Mol Med 1998:2:343-8.

451. Stocks JM, Wallace RJ Jr, Griffith DE, et al. Ofloxacin in community-acquired lowe respiratory infections. A comparison with amoxicillin or erythromycin. Am J Med 1989:87(6C):52-6S

452. Morandini G, Perduca M, Zannini G, et al. Clinical efficacy of azithromycin in lower respiratory tract infections. J Chemother 1993;5:32-6.

453. Finch RG, Woodhead MA. Practical considerations and guidelines for the management of community-acquired pneumonia. Drugs 1998;55:31-45.

454. Moreno S, Garcia-Leoni ME, Cercenado E, et al. Infections caused by erythromycinresistant Streptococcus pneumoniae: incidence, risk factors, and response to therapy in a prospective study. Clin Infect Dis 1995;20:1195-200.

455. Jackson MA, Burry VF, Olson LC, et al. Breakthrough sepsis in macrolide-resistant pneumococcal infection. Pediatr Infect Dis J 1996;15:1049-51.

456. Amsden GW. Pneumococcal macrolide resistance - myth or reality? J Antimicrob Chemother 1999:44:1-6. 
457. Perez-Trallero E. Pneumococcal macrolide resistance-not a myth. J Antimicrob Chemother 2000;45:401-3.

458. Carbon C, Poole MD. The role of newer macrolides in the treatment of communityacquired respiratory tract infection. A review of experimental and clinical data. J Chemother 1999;11:107-18.

459. Moss PJ, Finch RG. The next generation: fluoroquinolones in the management of acute lower respiratory infection in adults. Thorax 2000;55:83-5.

460. Robenshtok E, Shefet D, Gaafter-Gvili A, et al. Empiric antibiotic coverage of atypical pathogens for community acquired pneumonia in hospitalized adults. Cochrane Database Systemat Rev 2008;(1):CD004418.

461. Shefet $\mathbf{D}$, Robenshtok E, Paul M, et al. Empirical atypical coverage for inpatients with community-acquired pneumonia: systematic review of randomized controlled trials. Arch Intern Med 2005;165:1992-2000

462. Mills GD, Oehley MR, Arrol B. Effectiveness of beta lactam antibiotics compared with antibiotics active against atypical pathogens in non-severe community acquired pneumonia: meta-analysis. BMJ 2005;330:456.

463. Sportel JH, Koeter GH, van Altena R, et al. Relation between beta-lactamase producing bacteria and patient characteristics in chronic obstructive pulmonary disease (COPD). Thorax 1995;50:249-53

464. Meehan TP, Fine MJ, Krumholz HM, et al. Quality of care, process, and outcomes in elderly patients with pneumonia. JAMA 1997;278:2080-4.

465. Houck PM, Bratzler DW, Nsa W, et al. Timing of antibiotic administration and outcomes for Medicare patients hospitalized with community-acquired pneumonia. Arch Intern Med 2004;164:637-44.

466. Simpson JC, Macfarlane JT, Watson J, et al. A national confidential enquiry into community acquired pneumonia deaths in young adults in England and Wales. British Thoracic Society Research Committee and Public Health Laboratory Service. Thorax 2000:55:1040-5.

467. Marston BJ, Plouffe JF, File TM Jr, et al. Incidence of community-acquired pneumonia requiring hospitalization. Results of a population-based active surveillance Study in Ohio. The Community-Based Pneumonia Incidence Study Group. Arch Intern Med 1997;157:1709-18.

468. Houck PM, Bratzler DW, Nsa W, et al. Antibiotic administration in communityacquired pneumonia. Chest 2004;126:320-1.

469. Metersky ML, Sweeney TA, Getzow MB, et al. Antibiotic timing and diagnostic uncertainty in Medicare patients with pneumonia: is it reasonable to expect all patients to receive antibiotics within 4 hours? Chest 2006;130:16-21.

470. Welker JA, Huston M, McCue JD. Antibiotic timing and errors in diagnosing pneumonia. Arch Intern Med 2008:168:351-6.

471. Kanwar M, Brar N, Khatib R, et al. Misdiagnosis of community-acquired pneumonia and inappropriate utilization of antibiotics: side effects of the 4-h antibiotic administration rule. Chest 2007:131:1865-9.

472. Schulin T, Wennersten CB, Ferraro MJ, et al. Susceptibilities of Legionella spp. to newer antimicrobials in vitro. Antimicrob Agents Chemother 1998;42:1520-3.

473. Gleason PP, Meehan TP, Fine JM, et al. Associations between initial antimicrobial therapy and medical outcomes for hospitalized elderly patients with pneumonia. Arch Intern Med 1999:159:2562-72.

474. Burgess DS, Lewis JS 2nd. Effect of macrolides as part of initial empiric therapy on medical outcomes for hospitalized patients with community-acquired pneumonia. Clin Ther 2000:22:872-8.

475. MacGregor RR, Graziani AL. Oral administration of antibiotics: a rational alternative to the parenteral route. Clin Infect Dis 1997;24:457-67.

476. Mandell LA, Bergeron MG, Gribble MJ, et al. Sequential antibiotic therapy: effective cost management and patient care. Can J Infect Dis 1995;6:306-15.

477. Siegel RE, Alicea M. Lee A, et al. Comparison of 7 versus 10 days of antibiotic therapy for hospitalized patients with uncomplicated community-acquired pneumonia: a prospective, randomized, double-blind study. Am J Ther 1999;6:217-22.

478. Nathwani D. Sequential switch therapy for lower respiratory tract infections: a European perspective. Chest 1998;113(3 Suppl):211-8S.
479. el Moussaoui R, de Borgie CAJM, van den Broek $P$, et al. Effectiveness of discontinuing antibiotic treatment after three days versus eight days in mild to moderate-severe community acquired pneumonia: randomised, double blind study BMJ 2006:332:1355.

480. Pedro-Botet L, Yu VL. Legionella: macrolides or quinolones? Clin Microbiol Infect 2006; 12(Suppl 3):25-30.

481. Sabria M, Pedro-Botet ML, Gomez J, et al. Fluoroquinolones vs macrolides in the treatment of Legionnaires disease. Chest 2005;128:1401-5.

482. Blazquez Garrido RM, Espinosa Parra FJ, Alemany Frances L, et al. Antimicrobial chemotherapy for Legionnaires disease: levofloxacin versus macrolides. Clin Infect Dis 2005;40:800-6.

483. Mykietiuk A, Carratala J, Fernandez-Sabe N, et al. Clinical outcomes for hospitalized patients with Legionella pneumonia in the antigenuria era: the influence of levofloxacin therapy. Clin Infect Dis 2005;40:794-9.

484. Owens RC Jr, Nolin TD. Antimicrobial-associated QT interval prolongation: points of interest. Clin Infect Dis 2006;43:1603-11.

485. Grau S, Antonio JM, Ribes $\mathrm{E}$, et al. Impact of rifampicin addition to clarithromycin in Legionella pneumophila pneumonia. Int J Antimicrob Agents 2006;28:249-52.

486. Hubbard RB, Mathur RM, MacFarlane JT. Severe community-acquired legionella pneumonia: treatment, complications and outcome. $0 \mathrm{~J}$ Med 1993;86:327-32.

487. Kirby J, Polis G, Romansky MJ. A controlled blind study of pneumococcal pneumonia treated with tetracycline and tetracycline plus 6-methyl prednisolone. Antibiot Ann 1959:7:175-81.

488. Roson B, Carratala J, Fernandez-Sabe N, et al. Causes and factors associated with early failure in hospitalized patients with community-acquired pneumonia. Arch Intern Med 2004:164:502-8.

489. Genne D, Sommer R, Kaiser L, et al. Analysis of factors that contribute to treatment failure in patients with community-acquired pneumonia. Eur J Clin Microbiol Infect Dis 2006;25:159-66.

490. Menendez R, Torres A, Zalacain R, et al. Risk factors of treatment failure in community acquired pneumonia: implications for disease outcome. Thorax 2004:59:960-5.

491. Heffner JE, McDonald J, Barbieri C, et al. Management of parapneumonic effusions. An analysis of physician practice patterns. Arch Surg 1995;130:433-8.

492. Light RW. A new classification of parapneumonic effusions and empyema. Chest 1995; 108:299-301

493. Heffner JE, Brown LK, Barbieri C, et al. Pleural fluid chemical analysis in parapneumonic effusions. A meta-analysis. Am J Respir Crit Care Med 1995;151:1700-8

494. Ahmed RA, Marrie TJ, Huang JO. Thoracic empyema in patients with communityacquired pneumonia. Am J Med 2006;119:877-83.

495. Finley C, Clifton J, Fitzgerald JM, et al. Empyema: an increasing concern in Canada. Can Respir J 2008:15:85-9.

496. Farjah F, Symons RG, Krishnadasan B, et al. Management of pleural space infections a population-based analysis. J Thorac Cardiovasc Surg 2007;133:346-51.

497. Davies CW, Gleeson FV, Davies RJ. BTS guidelines for the management of pleural infection. Thorax 2003;58(Suppl 2):ii18-28.

498. Almirall J, Bolibar I, Serra-Prat M, et al. New evidence of risk factors for community-acquired pneumonia: a population-based study. Eur Respir J 2008:31:1274-84.

499. Baik I, Curhan GC, Rimm EB, et al. A prospective study of age and lifestyle factors in relation to community-acquired pneumonia in US men and women. Arch Intern Med 2000;160:3082-8.

500. Nuorti JP, Butler JC, Farley MM, et al. Cigarette smoking and invasive pneumococcal disease. Active Bacterial Core Surveillance Team. N Engl J Med 2000:342:681-9

501. West R, McNeill A, Raw M. Smoking cessation guidelines for health professionals: an update. Health Education Authority. Thorax 2000;55:987-99.

502. Berntsson E, Blomberg J, Lagergard T, et al. Etiology of community-acquired pneumonia in patients requiring hospitalization. Eur J Clin Microbiol 1985;4:268-72. 


\section{APPENDIX 1 CHECKLIST USED BY REVIEWERS FOR APPRAISING STUDIES}

Study:

Reviewer:

Please complete section 1 first. If study $0 \mathrm{~K}$, complete one of sections 2a-d as appropriate.

\section{General: is the study relevant to our question?}

$\square$ Were the patients studied similar (in age, gender, disease severity ...) to target patients?

$\square$ Were the outcome measures of interest to us and our patients?

$\square$ Was the clinical setting (primary care, intensive care ...) similar to our setting?

Was the study carried out in a healthcare system similar to ours?

Is the study design recognisable and appropriate, with clear methods described?

Is the study recent enough to take account of any important advances?

If negative, was this study large enough to provide useful information?

\section{2a. Studies of cause and effect (randomised trial of treatment)}

$\square$ Was assignment of patients to treatment truly randomised?

Was the planned therapy concealed from those recruiting patients before enrolment?

$\square$ Were all patients who entered the study accounted for?

$\square$ Were patients analysed in the groups to which they were initially randomised?

Were patients and doctors blind to the therapy given?

Were groups treated the same way, apart from the therapy?

Were the groups similar at the start of the trial?

2b. Studies of aetiology (case-control study of a harmful agent)

$\square$ Were there two groups of cases, similar except for exposure to harmful agent?

Was occurrence of the outcome measured in the same way for both groups?

$\square$ Were enough patients followed up for long enough for the outcome to develop?

Did exposure clearly precede the outcome?

Was there a dose-response gradient?

Was there a re-challenge, or improvement after the drug stopped?

Does the association make biological sense?

2c. Studies of diagnosis (evaluation of clinical findings or tests) $\square$ Was the finding or result compared with a 24 carat gold standard for diagnosis? Was the finding or result determined blind to the gold standard?

Was the gold standard determined blind to the finding or test result?

$\square$ Was the gold standard determined in all cases, not just those with an abnormal result?

\section{2d. Studies of prognosis, prognostic index (cohort studies)}

$\square$ Was a defined sample of patients assembled at an early stage of the disease? $\square$ Were patients followed up long enough for the outcome to develop?

$\square$ Was the outcome clearly defined, objective and assessed blind to exposure in all cases?

$\square$ Was the performance of any prognostic index tested on a fresh set of cases?

\section{Comments}

APPENDIX 2 ADDITIONAL CHECKLIST USED FOR APPRAISING STUDIES TO INFORM PNEUMONIA AETIOLOGY

Absolute requirements:

- Is this an original report?

- Were patients with CAP separately identified?

Was the study designed to assess CAP aetiology?

- Was the patient sample representative (eg, sufficient numbers, consecutive cases, exclusions clearly defined)?

- Was the study of sufficient duration to exclude seasonal bias?

Data qualification:

- Is the geographical area clear and relevant?

- Is the patient age group defined?

- Are microbial investigations clearly defined?

- Was the investigation biased towards a specific pathogen?

- Is the setting community, hospital, intensive care or a combination? 
Appendix 3 Types of study and levels of evidence used to illuminate specific clinical questions

\begin{tabular}{|c|c|c|c|}
\hline Type of clinical question & Evidence level & Brief definition & Types of study providing this level of evidence \\
\hline \multirow[t]{2}{*}{ Treatment } & la & A good recent systematic review & Systematic review of randomised trials \\
\hline & $\mathrm{lb}$ & A rigorous study designed to answer the question & A rigorous randomised trial comparing $T$ with best alternative \\
\hline \multirow[t]{4}{*}{ Is therapy T effective? } & II & One or more prospective clinical studies & A cohort study or faulty randomised trial \\
\hline & III & One or more retrospective clinical studies & A case-control study \\
\hline & $\mathrm{IVa}$ & Formal expert consensus & Delphi study of expert practice \\
\hline & $\mathrm{IVb}$ & Other information & Study of pharmacology of $\mathrm{T}$ \\
\hline Aetiology or harm & la & A good recent systematic review & Systematic review of cohort studies \\
\hline Does A cause disease D? & $\mathrm{lb}$ & A rigorous study designed to answer the question & A large well-designed cohort study \\
\hline \multirow[t]{4}{*}{ Does drug $D$ cause side effect $S ?$} & ॥ & One or more prospective clinical studies & A faulty cohort study \\
\hline & III & One or more retrospective clinical studies & A case-control study \\
\hline & $\mathrm{IVa}$ & Formal expert consensus & Delphi study of expert opinion \\
\hline & $\mathrm{IVb}$ & Other information & Study of pathophysiology of D \\
\hline Diagnosis or prognosis & la & A good recent systematic review & Systematic review of blind comparisons of $\mathrm{T}$ with gold standard \\
\hline $\begin{array}{l}\text { Is } T \text { an accurate test for diagnosis } \\
\text { of } D \text { ? }\end{array}$ & $\mathrm{lb}$ & A rigorous study designed to answer the question & $\begin{array}{l}\text { Blind prospective comparison of } \mathrm{T}, \mathrm{F} \text { or } \mathrm{M} \text { with gold standard for } \mathrm{D} \text { or } \mathrm{E} \\
\text { (eg, response to specific therapy) with multivariate analysis }\end{array}$ \\
\hline $\begin{array}{l}\text { Is finding } F \text { an accurate predictor } \\
\text { of event } E \text { ? }\end{array}$ & $\|$ & One or more prospective clinical studies & $\begin{array}{l}\text { Analysis of prospective test results in patients enrolled in a randomised } \\
\text { clinical trial of therapy for varying stages of } D \text {. Prospective validation } \\
\text { study with univariate analysis }\end{array}$ \\
\hline \multirow[t]{3}{*}{$\begin{array}{l}\text { Does severity measure } \mathrm{M} \\
\text { accurately predict event } \mathrm{E} \text { ? }\end{array}$} & III & One or more retrospective clinical studies & $\begin{array}{l}\text { Retrospective study of test results or findings in a database of patients } \\
\text { with univariate or multivariate analysis }\end{array}$ \\
\hline & $\mathrm{IVa}$ & Formal expert consensus & Delphi study of expert opinion about $\mathrm{T}$ \\
\hline & $\mathrm{IVb}$ & Other information & Study of pathophysiology of $D$ \\
\hline Public health, health policy & la & $\begin{array}{l}\text { Economic and policy analysis based on good } \\
\text { recent systematic reviews }\end{array}$ & $\begin{array}{l}\text { Economic and policy analysis with modelling and sensitivity analysis } \\
\text { using data from systematic reviews of effectiveness and of cost studies } \\
\text { in the same routine clinical settings }\end{array}$ \\
\hline \multirow[t]{5}{*}{$\begin{array}{l}\text { Is policy } P \text { cost effective in the } \\
\text { NHS? }\end{array}$} & $\mathrm{lb}$ & $\begin{array}{l}\text { Economic and policy analysis based on a rigorous } \\
\text { study designed to answer the question }\end{array}$ & $\begin{array}{l}\text { Economic and policy analysis with modelling and sensitivity analysis } \\
\text { using data from a randomised clinical trial of effectiveness and a cost } \\
\text { study in the same routine clinical setting }\end{array}$ \\
\hline & II & $\begin{array}{l}\text { Economic and policy analysis based on one or } \\
\text { more prospective clinical studies }\end{array}$ & $\begin{array}{l}\text { Economic and policy analysis with modelling and sensitivity analysis } \\
\text { using other prospective data in various settings }\end{array}$ \\
\hline & III & $\begin{array}{l}\text { Economic and policy analysis based on one or } \\
\text { more retrospective clinical studies }\end{array}$ & $\begin{array}{l}\text { Economic and policy analysis with modelling and sensitivity analysis } \\
\text { using retrospective data }\end{array}$ \\
\hline & $\mathrm{IVa}$ & Formal expert consensus & Delphi study of national expert opinion about $P$ \\
\hline & $\mathrm{IVb}$ & Other information & Local opinion about $\mathrm{P}$ \\
\hline
\end{tabular}

Appendix 4 Generic levels of evidence and guideline statement grades, appropriate across all types of clinical questions

\begin{tabular}{|c|c|c|c|}
\hline $\begin{array}{l}\text { Evidence } \\
\text { level }\end{array}$ & Definition & Example of study providing this level of evidence for a therapy question & $\begin{array}{l}\text { Guideline } \\
\text { statement grade }\end{array}$ \\
\hline la & $\begin{array}{l}\text { A good recent systematic review of studies designed to } \\
\text { answer the question of interest }\end{array}$ & $\begin{array}{l}\text { Cochrane systematic review of randomised controlled trials studying the } \\
\text { effectiveness of flu vaccines }\end{array}$ & $A+$ \\
\hline$\|^{*}$ & $\begin{array}{l}\text { One or more prospective clinical studies which illuminate, but } \\
\text { do not rigorously answer, the question }\end{array}$ & $\begin{array}{l}\text { Prospective cohort study comparing pneumonia rates in patients who are and } \\
\text { are not vaccinated against flu; non-randomised controlled trial }\end{array}$ & $\mathrm{B}+$ \\
\hline $\mathrm{IVb}$ & Other information & Expert opinion, informal consensus; in vitro or in vivo studies on related topics & D \\
\hline
\end{tabular}

*Hard to differentiate Agency for Health Care Policy and Research's "well designed controlled study without randomisation" (level lla) from "other type of well-designed experimental study" (level llb).

† Major criterion is retrospective versus prospective data collection, since non-experimental designs are better suited than even randomised clinical trials for answering certain questions.

\$Distinguish formal consensus from informal consensus methods according to the Health Technology Assessment 1998 systematic review. 